\title{
Lexicographical addenda
}

This chapter documents words and meanings not included in the standard Karaim dictionary, i.e. in the Karaim-Russian-Polish dictionary (KarRPS) published in 1974. Seen in this light, the present part of this book should be treated as a contribution to Karaim lexicography. It comprises native Karaim lexemes, loanwords, and idioms absent from KarRPS - including lexical items that appear in a different phonetic shape or with a different meaning than that lemmatized by the editors and authors of KarRPS. If, however, the phonetic difference is a result of regular historical sound changes or widespread tendencies that affected Karaim over the course of time, the word is not repeated here.

Every entry ends in a brief commentary (not etymology!) introduced by a "Latin Letter Ain" ( $\bullet$ ) symbol in which we provide, most importantly, the Modern Western Karaim comparative data and, in the case of loanwords and Fremdwörter, the etymons that are necessary to document each lexeme or meaning. It is perhaps important to note that when we searched for possible etymons, we took into consideration the historical variants of the donor languages. One example that readily comes to mind is the word blaha 'metal sheet' the possbile etymons of which, i.e. Ukr. блаха бляха 'metal sheet' and Pol. blacha id., were checked against Ukrainian and Polish historical dictionaries (in this case ISUJa and SStp.): we can safely say that the Polish word was first attested in the 15th-16th centuries, whereas Ukr. бляxa has been known since the $17^{\text {th }}$ century and thus both words could have been loaned into Karaim by 1720 . In the case of Hebrew, we took into account its Biblical variant when it was necessary to analyse phraseological, syntactic, semantic, or morphological calques. However, in some instances it was Medieval Hebrew that helped us understand the meaning of a wordsee, for instance, galgal 'celestial sphere'.

The glossary presents the respective forms of the lemmatized lexemes in the context they were used. In the contextualization the lemmatized forms have been abbreviated to their first letter, which allows us to determine precisely which form we are referring to in the glossary. A good example is the word ber- 'to give'-in Karaim religious texts it is often used in the meaning 'to make', as a semantic calque of Heb. נָתֵן '1. to give; 2. to permit; 3 . to deliver; 4 . to put, to set; 5 . to make' - which is attested twice in Gen 48:4: in the same future tense form (berirmen), but with two different meanings, see:

Da ajtty maja muna men jajamen seni da arttyryrmen seni da berirmen seni jyjynyna uluslarnyn da berirmen ošol ol jerni ošpunu urluġuja özüjden sortun tutuvlugiuna dunjanyn. [= And said to me, "Lo, I will spread you, and multiply you, and I will make of you a multitude of people, and will give this land to your offspring after you for an everlasting possession."] 
In its second occurrence in this verse, berirmen is used in its well-known meaning 'to give'. However, on the first occasion it is employed in the hitherto undocumented sense of 'to make'. Hence, in its glossary entry the word berirmen is only abbreviated to $b$. in its first occurrence.

We have not indicated in the glossary whether a certain word in a passage was added to the text on the margin or between lines if this was done by the main copyist. Marginal or interlinear additions by another hand are enclosed in curly brackets. Reconstructed portions of text are provided in square brackets.

Except for the names of feasts and holidays, proper nouns are not included in the glossary.

\section{A, 'A}

'aceret 'solemn assembly'

ol segizinči künd́a 'a. bolsun sizǵa heč qulluq iš qylmajyz (Num 29:35); alty künler ašaġyn macalar da ol jedinči künde 'a. Adonajġa Tenrije qylmag்yn iš (Deu 16:8)

-ti: juvutujuz otlu qarban Adonajg̉a 'a. ol heč qulluq iš qylmajyz (Lev 23:36)

\& Heb. עִ עָצר 'solemn assembly'.

ačyq 'bare (head)'

ol cara'atly ki anda ol hastalyq upraqlary bolsunlar sögülǵanlar da bašy bolsun a. (Lev 13:45)

e Mod.NWKar. ačyh 'open'.

ačyn- 'to uncover oneself'

-dy: ičti ol čaġyrdan da esirdi da a. ortasynda čatyrynyn (9:21)

e Mod.NWKar. ačyn- 'to open'.

adeple- 'to discipline, to punish'

-seler: bolsa kišige uvul azuvču da tanuvču tynlamavču sözüne atasynyn da sözüne anasynyn da a. any da tynlamasa alarġa (Deu 21:18)

-sinler: alsynlar qartlary ol šaharnyn ošol ol kišini da a. any (Deu 22:18)

e Mod.NWKar. hadepla- 'to instruct, to admonish', Mod.SWKar. adeple- 'to punish'. $\Rightarrow$ adepĺa-, adepĺan-

adepĺa- 'to discipline, to punish'

-jdi: bilgin aqylyjbyla ki nečik adeplajdir kiši ošol uvlun özünün alaj Adonaj Tenrij a. seni (Deu 8:5)

-jdir: bilgin aqylyjbyla ki nečik a. kiši ošol uvlun özünün alaj Adonaj Tenrij adeplajdi seni (Deu 8:5)

-ma: eger bularġadejin tynlamasajyz maja da arttyryrmen arttyryrmen a. sizni jedi anča jazyqlaryjyz üčün (Lev 26:18); köklard́an ešittirdi saja ošol ünün a. seni (Deu 4:36)

-rmen: jürürmen birǵajizǵa qahiribyla učurnun da a. sizni ham mend́a jedi anča jazyqlaryjyz üčün (Lev 23:28) 
e Mod.NWKar. hadepla- 'to instruct, to admonish', Mod.SWKar. adeple- 'to punish'. $\Rightarrow$ adeple-, adepĺan-

adeplan- 'to be disciplined, to be punished'

-maśajiz: eger bularbylada a. alnymda da jürüśajiz birǵaḿa učurbyla (Lev 26:23)

e Mod.NWKar. had́eplań- 'to be instructed, to be admonished', Mod.SWKar. adeplen-

'1. to learn; 2. to be punished'. $\Rightarrow$ adeple-, adepĺa-

ag̉ač: jemiš aġačy 'fruit tree'

bar ol aġačny ki andaj. a. urluqlavču urluq sizǵa bolsun jemǵa (Gen 1:19); ornatsajyz nendijdaj. a. (Lev 19:23); barmodu anda j. a.johmodu (Num 13:20); čejpamag்yn ošolj. a. anyn sunma anyn üstüne balta (Deu 20:19); ki tirliklaridi ol adamnyn j. a. ol tüznün (Deu 20:19)

e Mod.NWKar. ahač 'tree', Mod.NWKar. jemiś 'fruit'.

'ahbar 'mouse'

budur sizǵa ol murdar qumuz da ol tebránadog்an ol jer üstüńa ol holed da ol 'a. da ol cav žynsysajyn (Lev 11:29)

\& Heb. עַכְבְבר 'mouse'.

aḥer: aḥerindan 'in the course of (time), after (the time of )'

edi a. zamanlarnyn (Gen 4:3); edi a. qyrq künnün (Gen 8:6); a. onjyllarnyn (Gen 16:3); a. ekijyllarnyn (Gen 41:1); edi a. dört jüz da otuzjylnyn (Exo 12:41); passim

e Mod.SWKar. aheribe 'in the course of (time), after (the time of )'.

aḥlama 'amethyst'

ol üčünčü jerge lešem ševo da a. (Exo 28:19); ol üčünčü jerge lešem ševo da a. (Exo 39:12)

\& Heb. אַחְלָמָה 'the name of a precious stone, usually identified with the amethyst'.

aja 'a bird of prey (probably the falcon)'

-ny: ošol ol da’any da ošol ol a. ̌̌ynsysajyn (Lev 11:14); ol rajany da ošol ol a. da ol dajany з̌ynsysajyn (Deu 14:13)

e Heb. אָיָָ 'a bird of prey, probably the falcon', see Klein (1987: 21), Koehler \& Baumgartner (1985: 36).

ajip aji|p '1. nackedness; 2. private parts'

nendijd́a kiši bar juvuġuna gufnun juvumajyz aškartma a. (Lev 18:6); nendij kiši ... ki bolsa anda a. juvmasyn juvutma qarbanyn öz Tenrisinin (Lev 21:17); nendij kiši ki anda a. juvumasyn (Lev 21:18); nendijda kiši ki anda a. urlugiundan Aharonnun ol kohennin juvumasyn juvutma ošol otlu qarbanlaryn Adonajnyn a. anda ošol qarbanyn Tenrisinin juvmasyn juvutma (Lev 21:21); tek ol parohetḱk kelḿasin da ol mizbeaḥqa juvmasyn ki a. anda (Lev 21:23); passim

-|bij: da minmegin stupeńlerbyla anyn üstüne ki aškarmaġaj a. anyn üstüne (Exo 20:26)

-|bin: da kördü Ham atasy Kena'annyn [ošol] a. atasynyn (Gen 9:22); da qapladylar ošol a. atasynyn özleri[nin] da jüzleri alarnyn edilarartqary da a. atasynyn (Gen 9:23); čejsylavčulardyrsiz körme ošol a. ol jernin (Gen 42:9); ajtty alarġa joq ki a. ol jernin keldijiz körḿa (Gen 42:12) 
-idi: ajipin qatynynyn atajnyn aškartmaġyn a. atajnyn ol (Lev 18:8); aškartmag்yn a. qaryndašyjnyn ol (Lev 18:16)

-in: a. atajnyn da a. anajnyn aškartmag்yn anajdy ol aškartmaj̇yn a. anyn (Lev 18:7); a. qatynynyn atajnyn aškartmag்yn ajipidi atajnyn ol (Lev 18:8); a. tuvdug்ujnun qyzynyn atajnyn jemeśa qyzynyn anajnyn ... aškartmaġyn (Lev 18:9); a. qyzynyn uvlujnun [je]ḿaśa qyzynyn qyzyjnyn aškartmaġyn (Lev 18:10); a. qyzynyn qatynynyn atajnyn tuvdurġanyn atajnyn tuvdug்ujdu ol aškartmag்yn a. anyn (Lev 18:11); passim

-inden: ki urušsalar erenler birge kiši da qaryndašy anyn da juvusa qatyny ol birnin qutqarma ošol erin özünün qolundan vatuvčusunun da sunsa qolun da tutsa a. anyn (Deu 25:11)

-lerin: da keltirdi Josef ošol a. alarnyn jamanny (Gen 37:2) $\Rightarrow$-ĺarin

-larin: aškartmag்yn a. alarnyn (Lev 18:9); ajipin qyzynyn uvlujnun [ je] máá́a qyzynyn qyzyjnyn aškartmag்yn a. alarnyn (Lev 18:10) $\Rightarrow$-lerin

-nin: da qylgyn alarġa aq mihnesajimler qaplama etin a. bellerden (Exo 28:42)

e Mod.NWKar. ajip ajyp 'shame, disgrace; crime; villainy; defect, blemish'.

ajryč 'isolated'

olturdu a.jol qatyna ki jolu üstüńa Timnatnyn (Gen 38:14)

e Mod.SWKar. ajryc 'isolated, separate'.

ajtqan 'meaning, message'

-y: ol kiši ki ol aruvdu da jolda bolmasa ki ol jyraq jol a. oldu kim ki juvuqta bolsa ol sajlanġan orunga a kelalmása qylma qarbanyn ol pesahnyn vaġdasynda (Num 9:13)

e Mod.NWKar. ajt- 'to say'.

ajyryl-: ömčektan ajyryl- 'to wean (a baby)'

-dy: ulgajdy ol ulan da ö. a. (Gen 21:8)

-gan: da qyldy Avraham ullu ički ö. a. künd́a Jichaq (Gen 21:8)

e Mod.NWKar. ömćak 'breast', Mod.NWKar. ajyryl- 'to be separated'.

alajoq '1. as well as; 2 . in the same way, by the same token'

da ajtty Lavan kelgin alğy̌laġany Adonajnyn nek turasen tyšqaryda da men hadirledim ol üvnü a. orun hadirledim tevelerǵad́a (Gen 24:31); a. ki bolsa alarġa töre sözü keledilermaja (Exo 18:16); a. nevahtta ki keŕakli bolsa ajyrma arasyna ol qodešnin da arasyna ol alaj $[y]$ gn $[y] n$ da arasyna ol murdarnyn da arasyna ol aruvnun (Lev 10:10); a. ne vahtta ki kerakli bolsa üvratmía ulanlaryna Jisra'elnin (Lev 10:11); a. almajyz juluv andijdán kimǵa keŕaklidi qačma šaharyna miqlatynyn (Exo 35:32); hatna qylar Adonaj ošol jüregijni da ošol jüregin urlugijnun ki ol remezdi eksilmegine jecer hara'nyn anlyqbyla a. bolursen süvme qullugiun Adonaj Tenrijnin (Deu 30:6)

\& Mod.SWKar. alajok 'so, like that'. $\Rightarrow$ alajoqqa

alajoqqa '1. as well as; 2 . in the same way'

da qajsy ol kiši ki ornatty borlalyq da a. čag்yrmady any barsyn da qajtsyn üvüne mag்at öler čerüvde da özge kiši a. čyg்aryr any (Deu 20:6); qatyn kelešsej da özge kiši alyr any 
üv qondarsaj da olturmassen anda borlalyq or\{na\}tsaj da a. čyg்armassen any (Deu 28:30)

\& Mod.SWKar. alajok 'so, like that'. $\Rightarrow$ alajoq

anafa 'heron'

-ny: ošol ol hasidany ol a. ̌̌ynsysajyn da ošol duhifatny da ošol ol jary qanatyn (Lev 11:19); ol hasidany da ol a. ̌̌ynsysajyn da ol duhifatny da ol jary qanatyn (Deu 14:18)

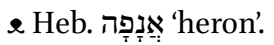

anaqa 'gecko'

ol a. da ol kovaḥ da ol leta’a da ol ḥomet da ol tinšamet (Lev 11:30)

\& Heb. אָנָָָּ 'gecko'.

aqšyn 'stomach'

-ny: bersin kohenge ol bilekni da ol jajaqlarny da ol a. (Deu 18:3)

-yna: čančty ošol eksilarind́a ošol Jisra’el kišini da ošol ol qatynny a. (Num 25:8)

e Mod.SWKar. aksyn 'stomach'.

arbe 'locust; a kind of locust'

köp urluq čyg்aryrsen ol tüzge da az jyštyryrsen ki tügetir any ol a. ündeledoġan šynsy čegirtkenin (Deu 28:38)

-ni: ošol bunlary alardan ašajyz ošol ol a. ̌̌ynsysajyn (Lev 11:22)

\& Heb. אַרְֶּ 'locust'.

aron '1. Ark; 2. coffin'

bergin ošol ol kaporetni ol a. üstüne jog்artyn (Exo 25:21); qaplag்yn ol a. üstüne ošol ol parohetni (Exo 40:3); qojdu ošol ol tutqučlarny ol a. üstüne (Exo 40:20); berdi ošol ol kaporetni ol a. üstüne joġartyn (Exo 40:20); alnyn ol kaporetnin ki ol a. üstüńa (Lev 16:2); passim

-da: qajyryldym endim ol tavdan da qojdum ošol ol luhotnu a. (Deu 10:5)

-ga: öldü Josef jüz da on jašar da hanot ettilar any da qojuldu a. Micrid́a (Gen 50:26); bergin ol a. ošol ol šarajatny (Exo 25:16); ol a. bergin ošol ol šarajatny ki bersem saja (Exo 25:21); aldy da berdi ošol ol šarajatny ol a. (Exo 40:20)

-nu: qylsynlar ošol ol a. šitim ag்ačlaryndan (Exo 25:10); eltme ošol a. (Exo 25:14); ošol ol a. šarajatqa (Exo 31:7); qyldy Becal'el ošol ol a. (Exo 37:1); eltme ošol ol a. (Exo 37:5); keltirdi ošol ol a. ol miškanġa (Exo 40:21); passim

-nun: janlary üstüne ol a. (Exo 25:14); kijdi ošol ol tutqučlarny jüzüklerge janlaryüstüne ol a. (Exo 37:5)

-u: eki keruvlarnyn ki a. üstüne (Exo 25:22); bergin ošol ol kaporetni a. üstüne ol šarajatnyn (Exo 26:34); alnynda ol parohetnin ki a. üstüne ol šarajatnyn (Exo 3o:6); qojdu ošol parohetin ol qaplavnyn da qaplady a. üstüne ol šarajtnyn (Exo 40:21); ol kaporet üstünd́an ki a. üstüńa ol šarajatnyn (Num 7:89); passim

-un: ošol a. ol šarajatnyn (Exo 39:35); qojġun anda ol a. ol šarajatnyn (Exo 40:3); jaġyndyrg்yn ošol ol qymg்anny da ošol a. anyn da aziz etkin any (Exo 40:11); qaplasynlar anyn byla ošol a. ol šarajatnyn (Num 4:5) 
-unun: bergin ošol altyn mizbeaḥny tütüge alnynda a. (Exo 40:5)

\& Heb. ארזון '1. box; 2. Ark; 3. coffin', Mod.SWKar. aron 'Ark of the Covenant'. arytqyč *cloth used for covering the showbread'

-lar: da ošol ol stolpeclerni ki alar üstüne edi ol a. ki qaplanyredi alarbyla arasyna har bir ötḿaknin ki barġaj jel ortalary ašyra alarnyn ki šemirlenmegej (Exo 37:16) -larny: da ošol ol qašyqlarny ki a larbyla ölčeredi ol kohen ošol ol tütünü da ošol a. (Exo 37:16); stolu üstüńa ol eki jüzlü ötḿaknin jajsynlar kök upraq da bersinlar anyn üstüńa ošol ol tepsilarni da ošol ol qašyq larny da ošol ol a. da ošol stolpeclarin ol qaplavyn da ol hammeśalik ötmák anyn üstüńa bolsun (Num 4:7)

-laryn: da qylg̈yn tepsilerin anyn da qašyqlaryn anyn da stolpeclerin anyn da a. anyn ki qaplandy alarbyla aruv altyndan qylgyn alarny (Exo 25:29)

e Perhaps related to Mod.WKar. aryt- 'to clean, to wash' or, less probably, to Mod.EKar. örtkič 'cover'. An explanation of arytqyč is provided in Exo 37:16: according to the translator, it was a cloth used for covering the space between showbreads to provide airflow between them preventing them from going mouldy, i.e. the cloth kept the bread clean (in religious sense). A similar explanation of Mod.NWKar. arythyc is provided in $\mathrm{R}\left(110 \mathrm{v}^{\mathrm{o}}\right)$ : ki jabulur alar byla 'which are used for covering'. Seen in this light, its relation to Kar. aryt- 'to clean' equipped with the -qyč derivative suffix that forms nomina instrumenti is somewhat more probable.

artyġač 'surplus; extra'

-ny: a. ol artadog்anny enlerin de ol čatyrnyn jarymyn ol ennin ol artadog்anny salg்yn artyüstüne ol miškannyn (Exo 26:12); da ošol bar ol a. qaldyryjyz özüjüzǵa saqlavg்a ol ertenbylaġadejin (Exo 16:23); javny ol qaplajdojanny ošol ol qarynny da ošol a. ol bavur üstüne (Exo 29:13); javny ki alar üstüńa ki ol qamču tïmaślar üstüńa da ošol ol a. ol bavurüstüńa ol bövŕakĺar byla ketarsin any (Lev 3:4); da ošol ol a. ol bavur üstüńa (Lev 3:10); passim

-yn: ošol ol javny ol qaplajdoginny ošol ol qarynny da ošol a. ol bavurnun (Exo 29:22); aldy ošol bar ol javny ki ol qaryn üstüńa da ošol a. ol bavurnun (Lev 8:16)

e Mod.NWKar. artyhač 'too much, too', Mod.SWKar. artyhac 'surplus'.

aslam 'profit, interest'

bolmag்yn anyn borčqa berüvčü kibik qojmajyz anyn üstüne a. (Exo 22:24); almag்y andan a. da fajda da qorqqun Tenrïizd́a da tiri bolsun qaryndašyj birǵaja (Lev 25:36); ošol kümüšüjnü berḿagin anar a. byla da fajda byla bermagin bürtügüjnü (Lev 25:37)

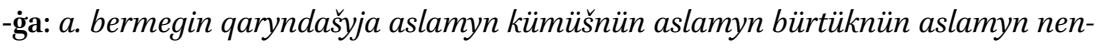
dijde nersenin ki a. beriledi (Deu 23:20); jatqa a. bergin da qaryndašyja a. bermegin (Deu 23:21)

-yn: aslamġa bermegin qaryndašyja a. kümüšnün a. bürtüknün a. nendijde nersenin ki aslamġa beriledi (Deu 23:20)

e Mod.EKar. aslam 'gain, profit'.

astrygadejin 'exceedingly, extremely'

qaltradyJichaqullu qaltravuqa. kiaškarmady anarbunerśa navilikašyra (Gen 27:33); 
ešitkečoq 'Esav ošol sözlerin atasynyn da firjat etti ullu firjat da ačy a. da ajtty atasyna alg̀yšlag̀yn menid́a dag்yn mend́a uvlujmen atam (Gen 27:34)

\& Mod.SWKar. astryhadejin 'exceedingly, extremely'.

ašam 'guilt offering'

biširilmásin ḥamec ülüšlarin alarnyn berdim any otlu qarbanlarymdan qodeš qadašimdir ol hatat kibik da a. kibik (Lev 6:10); nečik hatat alaj a. bir üvŕatüv alarg̉a (Lev 7:7); hatat kibikti ol ašam (Lev 14:13)

-g்a: budur ol üvŕatüv olaġa da tirkiǵa da hatatqa da a. da milu’imǵa da debehasyna ol šelamimnin (Lev 7:37); keltirsin qozu jyllyq balasy a. (Num 6:12)

-lary: bolsun saja qodešindán ol qadašimnin ol ottan bar qarbanlarysajyn bar tirkilarisajyn da bar hatatatlarysajyn da bar a. sajyn ki qajtarsalar maja qodeš qadašimdi saja ol da uvullaryja (Num 18:9)

-nyn: alsyn bir qozu qarbanyn a. tenufaġa bošatlyq qolma özü üčün (Lev 14:21)

\& Heb. אָָָ 'guilt offering'.

ašera 'Asherah, idol pole'

ornatmag்yn özüje a. heč ag்ačtan mizbeahy qatyna Adonaj Tenrijnin ki qylsaj özüje (Deu 16:21)

-laryn: ančaq ošol mizbeaḥlaryn alarnyn qavšatyjyz da ošol macevalaryn alarnyn syndyryjyz da ošol a. alarnyn kesijiz (Exo 34:13); ančaq bulaj qylyjyz alarġa mizbeahlaryn alarnyn qavšatyjyz da macevalaryn alarnyn syndyryjyz da a. alarnyn uvaltyjyz da jonma abaqlaryn alarnyn küvdürüjüz otta (Deu 7:5); qavšatyjyz ošolmizbeahlaryn alarnyn da syndyryjyz macevalaryn alarnyn da a. alarnyn küvdürüjüz otta da jonma abaqlaryn uvaltyjyz da tasetijiz ošol atlaryn alarnyn ol orundan (Deu 12:3)

\& Heb. אִשְׁרָר 'Asherah; name of a sacred tree or pole'.

ašağyšlyq 'haste'

da bulaj ašajyz any bellerijiz bolsunlar bajlanġanlar etiklerijiz bolsunlar ajaqlaryjyz üstüne da tajaġyjyz qolujuzda da ašajyz any a. byla qarbanydy Pesahnyn ol Adonajg்a (Exo 12:11); ašamag்yn anyn qatyna qužur ötmek jedi künler ašag்yn anyn qatyna macalar qyjynly ötmek ki a. byla čyqtyj (Deu 16:3)

e Mod.EKar. ašyg $y$ šlyq 'haste', Mod.NWKar. ašyh - 'to haste'. The - $a$ - of the second syllable is perhaps a scribal error in both instances-possibly influenced by aša- 'to eat' used in both verses in which the lemmatized word appears.

ašyq- 'to panic'

-majyz: ajtsyn alarġa tynlaġyn e Jisra’el siz juvujsiz bügün čerüvge dušmanlaryjyz üstüne jymšalmasyn jüregijiz qorqmajyz da a. da synyqmajyz alynlaryndan alarnyn

(Deu 20:3)

\& Mod.NWKar. ašyh ' 'to hurry'.

avaz '1. thunder; 2. rumour'

a. ešitildi üvünde par'onun (Gen 45:16)

-lar: sundu Moše ošol tajaġyn ol köklerge da Adonaj berdi a. da buz da čaj̇yldy ot jerge 
da javdurdu Adonaj buz jeriüstüne Micrinin (Exo 9:23); tefle etijiz Adonajġa da jetsin bolmaqtan ullu a. da buz da ijejim sizni da arttyrmajyz tohtama (Exo 9:28); čyqqanymdačoq ol šahardan jajarmen uvučlarymny alnynda Adonajnyn ol a. qalyrlar da ol buz bolmasty artyq anyn üčün ki bilgejsen ki Adonajnyn erkindedi ol jerde (Exo 9:29); da jajdy uvučlaryn alnynda Adonajnyn da qaldylar ol a. da ol buz da jamg்ur tammady jerǵa (Exo 9:33); kördü par'o ki qaldy ol jamg்ur da ol buz da ol a. da arttyrdy jazyqly bolma da qattyrdy jüregin ol da qullary anyn (Exo 9:34); boldu a. da jyldyrymlar da küčlü bulut ol tav üstüne da šofar avazy küčlü astry (Exo 19:16)

-larny: bar ol ulus körerediler ošol ol a. da ošol ol küsövlerni da ošol ol šofar avazyn (Exo 20:18)

e Mod.NWKar. avaz 'voice, sound', BHeb. קוֹ '1. voice, sound; 2. thunder; 3. rumour'.

avlama|q 'game, quarry (animal hunted)'

süvdü Jichaq ošol 'Esavny ki a. beriredi avzuna anyn (Gen 25:28); alġyn endi savutlaryjny qylyčyjny da jajny da čyqqyn ol tüzǵa da avlaġyn maja a. (Gen 27:3); Riveqa ešitiredi sözlegend́a Jichaq 'Esavg்a uvluna da bardy 'Esav ol tüzǵa avlama a. keltirḿa (Gen 27:5); keltirgin maja a. da qylg̀yn tatly ašlar (Gen 27:7); kim edi bunda ol avlavču a. (Gen 27:33); ol tirilüvčü ortajyzda ki avlasa a. kijik jemeśa quš (Lev 17:13)

-|'̇yndan: juvutqun maja da ašajym a. uvlumnun (Gen 27:25); tursun atam da ašasyn a. uvlunun (Gen 27:31)

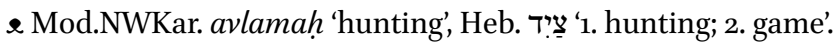

avlaq et- 'to make room'

-ti: da ajtty ki haligińa a. e. Adonaj bizǵa da jajylyrbiz jerd́a (Gen 26:22)

^ Mod.NWKar. avlah 'broad, spacious' + aux. verb.

avnet 'girdle'

bulardylar ol upraqlar ki qylsynlar ḥošen da efod da qaftan da ojuvlu kölmek micnefet da a. da qylsynlar aziz upraqlar Aharonġa qaryndašyja da uvullaryna anyn kohenlik etme maja (Exo 28:4); da a. qylġyn išin naġyšlavčunun (Exo 28:39); bajlandyrg்yn alarny a. byla (Exo 29:9); bajlandyrdy any a. byla (Lev 8:7); bajlandyrdy alarny a. byla (Lev 8:13); aq a. byla bajlansyn (Lev 16:4)

-ler: da qylgynn alarġa a. (Exo 28:40)

-ni: ošol ol a. ešken bezden da kökten da jipkinden da qyrmyzy jipekten iši nag்yšlavčunun (Exo 39:29)

\& Heb. אַבְנְּ 'girdle'.

avuz: qylyč avzu 'the edge of a sword'

-ndan: ošol Hamornu da ošol Šehemni uvlun anyn öltürdüler q. a. da aldylar ošol Dinany (Gen 34:26); qyrdy Johošua' ošol 'Amaleqni da ošol ulusun anyn q. a. (Exo 17:13); qyrdy any Jisra’el q. a. (Num 21:24); veren etkin any da ošol barča neki anda da ošol tuvaryn anyn q. a. (Deu 13:16); da qyrg்yn ošol bar erkegin anyn q. a. (Deu 20:13)

e Mod.NWKar. kylyč 'sword', Mod.NWKar. avuz 'mouth'. 
avuzluqla- 'to muzzle'

-mag̀yn: a. ögüznü bürtükjančqanynda (Deu 25:4)

e Mod.SWKar. avuzluk 'muzzle', Mod.NWKar. avuzluh 'bridle'.

az: azar azar 'little by little'

a. a. sürermen any alnyjdan neginče ki jajylġajsen da ülüš algajsen ošol ol jerni (Exo 23:30); süńar Adonaj Tenrij ošol ol hanlyqlarny ošpularny alnyjdan a. a. bolalmassen tavusma alarny tez mag்at artar üstüja kijigi ol tüznün (Deu 7:22)

e Mod.NWKar. az 'little' + deriv. suff.

aż̇yr- 'to prostitute (to make somebody a prostitute)'

-ma: jengil etŕagin qyzyjny a. any da azmasyn eli ol jernin da tolar ol jer jiŕanči išlard́an (Lev 19:29)

-yrlar: da a. ošol uvullaryjny qullug்u artyna abaqlarynyn özlerinin (Exo 34:16)

e Mod.NWKar. $a z$ - 'to prostitute oneself, to fall into harlotry', Mod.NWKar. azhyr- '1. to mislead; 2. to tempt'

aziz tut- 'to hallow'

-ujuz: a. t. ošol jylyn ol enlinči jylnyn da čaġyryjyz azatlyq jerd́a bar olturuvčularyna anyn joveldir ol (Lev 25:10)

e Mod.NWKar. aziź 'holy' + aux. verb.

'aznija 'black vulture'

-ny: da ošol bunu jiŕanči etijiz ol quštan ašalmasynlar qusqunčudur alar ošololnešerni da ošol ol peresni da ošol ol 'a. (Lev 11:13); budur ki ašamajyz alardan ol nešerni da ol peresni da ol 'a. (Deu 14:12)

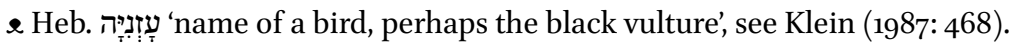

azuvču 'stubborn'

ki bolsa kišige uvul a. da tanuvču tynlamavču sözüne atasynyn da sözüne anasynyn (Deu 21:18); da ajtsynlar qartlaryna šaharynyn uvlumuz bu a. da tanuvčudu tynlamajdy sözümüzge (Deu 21:20)

e Mod.NWKar. azuvču '1. harlot; 2. criminal'.

aždaha *' 1 . sea creature; 2 . serpent'

-ga: alġyn ošol tajag்yjny da tašlag்yn alnynda par'onun bolsun a. (Exo 7:9); da tašlady Aharon ošol tajaġyn ... da boldu a. (Exo 7:10);

-larġa: tašladylar kiši tajaġyn özünün da boldular a. (Exo 7:12)

-larny: jaratty Tenri ošol ol a. o[l ullular]ny da ošol ol bar tiri žanny ol tebrenedoġanny [ki qoz] ladylar ol suvlar (Gen 1:21)

-larnyn: uvu a. ča ġyrlary alar[nyn] da uvu šavag்atsyz a. (Deu 32:33)

e Mod.NWKar. ačdaha aždaha 'dragon'. 
B

balqy- 'to shine'

-dy: ol qujaš b. ol jer üstüna da Lot keldi Co'arġa (Gen 19:23); b. anar ol qujaš ki nečik ašty ošol Penu’elni (Gen 32:32); da Moše bilmedi ki b. terisi jüzlerinin sözlegeninde birgesine (Exo 34:29); kördü Aharon da bar ulanlary Jisra’elnin ošol Mošeni da muna b. terisi jüzlerinin (Exo 34:30); körerediler ulanlary Jisra’elnin ošol jüzlerin Mošenin ki b. terisi jüzlerinin Mošenin (Exo 34:35); ajtty šehinasy Adonajnyn Sinaj sartyn keldi da b. balquvu šehinanyn Se ir sartyn (Deu 33:2)

-sa: eger b. anyn üstüne ol qujaš (Exo 22:2)

\& Mod.EKar. balqy- 'to shine'.

baqma 'watchtower'

ol b. ki ajtty baqqaj Adonaj arama da araja ki jašynsaq kisi dostundan (Gen 31:49)

e Mod.NWKar. bah- 'to watch' + deriv. suff.

bašqa 'separately'

da qojdu özüńa böleklar b. da qojmady alarny qoju qatyna Lavannyn (Gen 30:40); berdi qoluna qullarynyn birer bölek b. da ajtty qullaryna ašyjyz alnymda (Gen 32:17); qojdular anar b. da alarġa b. da Micrililerǵa ol ašavčularġa birgesińa b. ki bolalmasedilar ol Micrililer ašama Jisra’ellerbyla ötmek ki jerinčilik edi ol Micriǵa (Gen 43:32); qošqun ošol beš ol enlerni b. da ošol alty ol enlerni b. (Exo 26:9); alġyn özüje otjamlar musk da revent da galban otjamlar da aruv levona b. b. har birisi bolsun (Exo 30:34); qoštu ošol beš ol enlerni b. da alty ol enlerni b. (Exo 36:16)

e Mod.NWKar. bašha '1. other; 2. postp. except, besides'.

beklevüč '1. border, rim; 2. bar'

qylġyn anar b. tutam čüvre da qylg்yn altyn quršov beklevüčüne anyn čüvre (Exo 25:25); ortančy b. ortasynda ol tahtalarnyn (Exo 26:28); qyldy anar b. tutam čüvre da qyldy altyn quršov beklevüčüne anyn čüvre (37:12)

-ler: beš b. tahtalaryna ol ekinči janynyn ol miškannyn (Exo 26:27); beš b. ol ma'aravsarg்y janyna ol miškannyn qyryjlarġa (Exo 26:27); qyldy b. šitim ag்ačlaryndan bešni ol bir janyna ol miškannyn (Exo 36:34); beš b. taḥtalaryna ol miškannyn qyryjlarda ma'aravsary (Exo 36:32)

-lerge: qylgyn altyndan orunlar b. (Exo 26:29)

-lerin: ošol tahtalaryn anyn da ošol b. anyn ošol baġanalaryn anyn da ošol tabanlaryn anyn (Exo 35:11); anyn b. da baġanalary da tabanlary anyn (Exo 39:33); da berdi ošol b. anyn da turg்uzdu ošol baġanalaryn anyn (Exo 40:18)

-lerni: ošol jüzüklerin alarnyn qyldy altyndan orunlar beklavüčlarǵa da qaplady ošol ol b. altyn byla (Exo 36:34)

-nü: qyldy ošol ol ortančy b. bekleme ortasynda ol taḥtalarnyn ol učtan ol učqa (Exo 36:33)

-nün: uturusuna ol b. bolurlar ol jüzükler orunlar tutqučlarġa eltme ošol ol stolnu (Exo 25:7) 
-üne: qylġyn anar beklevüč tutam čüvre da qylg்yn altyn quršov b. anyn čüvre (Exo 25:25); qyldy anar beklevüč tutam čüvre da qyldy altyn quršov b. anyn čüvre (37:12)

e Mod.NWKar. beḱlavič beḱlav́uć 'bolt; lock', Mod.SWKar. beklevic 'barrier; arm, railing. Spelled ביכְלִיוביוּי beklüvüč was changed by the copyist into beklevüč during the process of vocalization. $\Rightarrow$ bekĺavüč

beklavüč 'border, rim'

-ĺarǵa: ošol jüzüklerin alarnyn qyldy altyndan orunlar b. da qaplady ošol ol beklevüčlerni altyn byla (Exo 36:34)

e Mod.NWKar. beḱlavič́ beḱlav́uć 'bolt; lock', Mod.SWKar. beklevic 'barrier; arm, railing'. $\Rightarrow$ beklevüč

belgirt- '1. to declare; 2 . to determine'

-me: köčüvünde ol Jardennin jerinde Mo’avnyn küvüllendi Moše b. ošol ol Torany ošpunu ajtadog்ač (Deu 1:5); jazg்yn ol tašlarüstüne ošol bar sözlerin ol Toranyn ošpunun b. jaḩ̌y (Deu 27:8) $\Rightarrow$-ma

-ḿa: qojdular any saqlavda b. alarġa nendij ölüm byla öltürḿa any bujrug் byla Adonajnyn (Lev 24:12) $\Rightarrow$-me

e Mod.NWKar. beĺgirtil- 'to be declared'.

belma 'cataract'

jemeśa bükrü jemeśa uvaq jemeśa b. közünd́a jemeśa qyršang்y jemeśa qyng்yrajġan jemeśa šiškan ajipli (Lev 21:20)

e Pol. bielmo 'cataract', Russ. бельмо id.

ber- '1. to set, to put, to place; 2 . to make; 3 . to allow'

-di: aldy özüńa ošol bar bularny da jartylady alarny ortada da b. kišinin jartysyn uturusuna dostunun da ošol qušnu jartylamady (Gen 15:10); sözledi ol kiši biji ol jernin birgemizǵa qaty sözlar da b. bizni čejsylavčularny kibik ošol ol jerni (Gen 42:30); $b$. Adonaj ošol širinligin ol ulusnun közlerinde elinin Micrinin (Exo 11:3); sajlady Moše tuvušlu elni bar Jisra'elden da b. alarny aġaraq ol ulus üstüne (Exo 18:25); b. anyn üstüńa ošol ol kölḿakni (Lev 8:7); ki ol hanlyqlar ošpular ki sen tasetesen alarny sahatny sajlavčularġa da qusnačylyq etüvčülerge tynlarediler da sen tüvül alaj b. saja Adonaj Tenrij (Deu 18:14)

-diler: ošol eki učlaryn eki ol örkenlernin b. eki ol ojuvlarüstüne da berdiler javrunlary üstüne ol efodnun uturusuna jüzlerinin (Exo 39:18); b. anyn üstüne kök šnur berme ol micnefet üstüne joġartyn ki nečik bujurdu Adonaj Mošege (Exo 39:31)

-dim: kör b. seni malah ornuna paróga (Exo 7:1); ol tirlikni da ol ölümnü b. alnyjda (Deu 3o:19)

-gin: b. ary tolu 'omer ündeledog்an ölčüvnü man da qojg்n any alnynda Adonajnyn saqlavġa (Exo 16:33); b. ol arong் ošol ol šarajatny ki bersem saja (Exo 25:16); b. ošol ol parohetni ol eslar tübüne (Exo 26:33); b. ošol eki altyn örkenlerni eki ol jüzükler üstüne (Exo 28:24); ošol eki učlaryn eki ol örkenlernin b. eki ol ojuvlar üstüne da b. javrunlary 
üstüne ol efodnun utrusuna jüzlerinin (Exo 28:25); da b. ošol ol altyn tačny ol micnefet üstüne (Exo 29:6); qylg̀yn qumġan tučtan da ornun anyn tučtan juvunmaqqa da b. any ohel mo'ed byla mizbea ḥ arasyna da b. ary suv (Exo 30:18); b. ošol qaplavyn qabag்ynyn ol qạ̣rany (Exo 40:8); b. anyn üstüńa jav da qojg̉un anyn üstüńa levona tirkidi ol (Lev 2:15); alġyn ošol ol bizni da b. qulaġyna anyn da ešikke (Deu 15:17)

-ip: ajtty anar Moše künülajmusen sen menim üčün da kim b. barda ulusu Adonajnyn bolsajdylar navilar ki berśajdi Adonaj ošol alhemin alar üstüna (Lev 11:29)

-ir: turalmasty kišide alnyjyzda qorquvujuznu da qobuvujuznu b. Adonaj Tenrijiz jüzleri üstüne bar ol jernin ki bassajyz anda ki nečik sözledi sizge (Deu 11:25)

-irmen: b. seni hanlyqlarġa da bijler send́an čyg்arlar (Gen 17:6); muna men jajamen seni da arttyryrmen seni da b. seni jyjynyna uluslarnyn (Gen 48:4); b. ošol širinligin ol ulusnun ošpu közlerinde Micrinin (Exo 3:21); b. ošol hyššymymny Micride (Exo 7:4) $\Rightarrow$-irḿan

-irman: ündelmesti artyq atyj senin Avram da bolur atyj senin Avraham ki atasy jyjynynyn hanlyqlarnyn b. seni (Gen 17:5) $\Rightarrow$-irmen

-me: b. üstüjüzge bügün alġyš (Exo 32:29); berdiler anyn üstüne kökšnur b. olmicnefet üstüne joġartyn ki nečik bujurdu Adonaj Mošege (Exo 39:31); qojg்n üstüje bij bolalmag்yn b. üstüje jat kišini (Deu 17:15)

-sejedi: kim b. ölmegimizni (Exo 16:3) $\Rightarrow$-śajdi, -śajedi

-sin: ol qaldyq javdan ki uvuču üstüńa b. ol kohen jymšaq ornu üstüńa ol on jandag்y qulaġynyn (Lev 14:17); ol qalġanny javdan ki uvuču üstüna ol kohennin b. bašy üstüna ol arynuvčunun (Lev 14:18)

-śajdi: ajtty anar Moše künülajmusen sen menim üčün da kim berip barda ulusu Adonajnyn bolsajdylar navilar ki b. Adonaj ošol alhemin alar üstüńa (Lev 11:29) $\Rightarrow$-sejedi, -śajedi

-śajedi: kim b. da bolsajedi (Deu 5:29) $\Rightarrow$-sejedi, -śajdi

\& ModNWKar. ber- 'to give'; the appearance of other meanings is influenced by Heb.

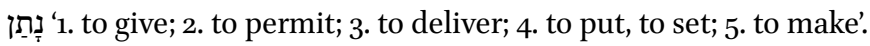

beri 'here; this way, here'

dörtünčü dor qajtyrlar b. ki tügenmedi günehi ol Emorinin ol vahtqa dejin (Gen 15:16); bunun byla synalyjyz tirlikleri üčün par'onun eger čyqsajyz bundan ki ančaq kelgend́a qaryndašyjyz ol kičirek b. (Gen 42:15); haligine ačyrg்anmajyz da ačuv bolmasyn közlerijizd́a ki sattyjyz meni b. ki beslenmekke sizǵa ijdi meni Tenri alnyjyzda (Gen 45:5); haligina tüvül siz ijdijiz meni b. ki ančaq ol Tenri da qojdu meni ata ornuna paŕoga (Gen 45:8); žaḥtlajyz da endirijiz ošol atamny b. (Gen 45:13); Tenri juvumaġyn b. (Exo 3:5)

-ge: men da ol ulan baryrbiz b. dejin da bašururbiz da qajtyrbiz sizǵa (Gen 22:5); čyqty ol birisi qatymdan da ajttym tek butarlanma butarlandy da körmedim any $b$. dejin (Gen 44:28)

e Mod.NWKar. bera 'this way, here', Mod.SWKar. beri 'here; here, in this direction'. 


\section{berrek 'this way'}

juvudular ol qaravašlar b. da ulanlary alarnyn da bašurdular (Gen 33:6)

e Mod.NWKar. beŕak 'this way, here', see A. Zajączkowski (1932: 48).

\section{bet hamiqdaš 'temple'}

barma tavyna ol Morijanyn ki anda bolur qondarylgan $b . h$. da anda debeha eterler rast debehalar (Deu 33:19)

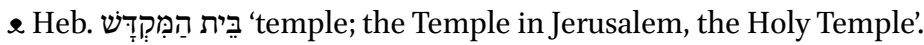

bez 'linen'

da kijdirdi anar b. upraqlar (Gen 41:42); da kök da jipkin da qyrmyzy jipek da b. da junun ečkilernin (Exo 25:4); ojuvlanġyn ol kölmekni b. byla (Exo 28:39); kök da jipkin da qyrmyzy jipek da b. da junun ečkilernin (Exo 35:6); bar kiši ki tabuldu birgesine kök da jipkin da qyrmyzy jipek da b. da junun ečkilernin (Exo 35:23); toldurdu alarny usubyla jüreknin qylma bar išin ustanyn da ojuvlavčunun da naġyšlavčunun kökbyla da jipkinbyla qyrmyzy jipekbyla b. byla (Exo 35:35); passim

-den: ošol ol miškanny qylg̈yn on enler ešken b. da kökten da jipkinden da qyrmyzy jipekten (Exo 26:1); qylg̀yn parohet kökten da jipkinden da qyrmyzy jipekten da ešken b. išin ojuvlavčunun (Exo 26:31); qyrmyzy jipekten da ešken b. iši naġyšlavčunun (Exo 26:36); ešken b. jüz lokot byla uzunluq (Exo 27:9); qaplav egirmi lokot kökten da jipkinden da qyrmyzy jipekten da ešken b. iši naġyšlavčunun (Exo 27:36); passim -ni: alsynlar ošol ol altynny da ošol ol köknü da ošol ol jipkinni da ošol ol qyrmyzy jipekni da ošol ol b. (Exo 28:5); da ošol ol b. (Exo 35:25)

e Mod.SWKar. beż 'textile, fabric', Mod.EKar. bez id. In the Hebrew Bible, its equivalent is Heb. نשׁ '(Egyptian) linen'.

bezirgen- 'merchant'

-ǵa: kümüš ašadog்an b. (Gen 23:16)

-lar: aštylar Midjanly erenlar b. (Gen 37:28)

e Mod.NWKar. beźarǵań 'merchant', Mod.SWKar. bezirgen 'merchant'.

bij 'king'

bulardylar ol bijler ki bijlikettilar jerind́a Edomnun b. bolmastan burun b. ulanlarynda Jisra’elnin (Gen 36:31); b. boldu Edomda Bela' (Gen 36:32); öldü Bela' da b. boldu anyn ornuna Jovav (Gen 36:33); öldü Jovav da b. boldu anyn ornuna Hušam (Gen 36:34); öldü Hušam da b. boldu anyn ornuna Hadad (Gen 36:35); öldü Hadad da b. boldu anyn ornuna Samla (Gen 36:36); passim

-i: qyldylar čerüv Bera' byla b. byla Sedomnun da Birša'byla b. byla 'Amoranyn Šinav byla b. byla Admanyn da Šemever byla b. byla Cevojimnin da bijibyla Bela'nyn oldur Co'ar (Gen 14:2); čyqty b. Sedomnun da b. 'Amoranyn da b. Admanyn da b. Cevojimnin da b. Bela'nyn oldu Co'ar (Gen 14:8); Kedarla'omer byla b. byla 'Elamnin da Tid'al byla b. byla Gojimnin da Amrafel byla b. byla Šin'arnyn da Arjoh byla b. byla Elasarnyn dört bijlarol bešbyla (Gen 14:9); qačtylar b. Sedomnun (Gen 14:10); čyqty b. Sedomnun uturusuna anyn (Gen 14:17); passim 
-in: qyrg்anyndan sortun ošol Siḥonnun b. ol Emorinin ki oltururedi Hešbonda ošol 'Oġnu b. ol Bašannyn ki oltururedi 'Aštarotta Edre ide (Deu 1:4); ošol Sihonnu b. Hešbonnun (Deu 2:24); dag்yn ošol 'Og்nu b. ol Bašannyn (Deu 3:3)

-ine: b. Micrinin (Exo 3:18); paróga b. Micrinin (Exo 6:13); par'oga b. Micrinin (Exo 6:27); par'oga b. Micrinin (Exo 6:29); anlatyldy b. Micrinin (Exo 14:5); passim $\Rightarrow$-ińa -inin: edi vahtlarynda Amrafelnin b. Šinarnyn Arjo[h]nun b. Elasarnyn Kedarla'omernin b. 'Elamnyn da Tid'alnyn b. Gojimnin (Gen 14:1); b. Micrinin (Gen 40:1); ötmek biširüvčü ki bijinin Micrinin (Gen 40:5); sordu vezirlerindán \{par'onun\} ki birgesińa saqlavynda üvünün bijinin (Gen 40:7); paronun b. Micrinin (Gen 41:46); passim -ińa: ajtty Avram b. Sedomnun (Gen 14:22); b. Micrinin (Gen 40:1); Avimelehḱa b. Pelištimnin (Gen 26:1); b. Micrinin (Gen 40:1); sözlegin par'oga b. Micrinin (Exo 6:11); passim $\Rightarrow$-ine

-ler: on dörtünčü jylda keldi Kedarla'omer da ol b. ki birgesina (Gen 14:5); bulardylar ol b. ki bijlik ettilar jerind́a Edomnun (Gen 36:31) $\Rightarrow$-lar

-lerine: ki nečik qyldy Sihonġa da 'Og்ja b. ol Emorinin (Deu 31:4)

-lerni: čyqty biji Sedomnun uturusuna anyn qajtqanyndan sortun qyrmaqtan ošol Kedarla'omerni da ošol ol b. ki birgesińa enišińa Šavenin oldu eniši ol bïnin (Gen 14:17) -lar: Kedarla'omer byla bijibyla 'Elamnin da Tid'al byla bijibyla Gojimnin da Amrafel byla bijibyla Šin'arnyn da Arjoh byla bijibyla Elasarnyn dört b. ol beš byla (Gen 14:9) $\Rightarrow$-ler

-larǵa: eki ol b. (Deu 3:21)

-larin: berir b. alarnyn qoluja (Deu 7:24)

-ĺarinin: qolundan eki b. ol Emorinin (Deu 3:8); eki b. ol Emorinin (Deu 4:47)

-nin: čyqty biji Sedomnun uturusuna anyn qajtqanyndan sortun qyrmaqtan ošol Kedarláomerni da ošol ol bijlerni ki birgesińa enišińa Šavenin oldu eniši ol b. (Gen 14:17); jolubyla ol b. baryrbiz (Num 20:17); jolubyla ol b. baryrbiz (Num 21:22);

e Mod.NWKar. bij 'lord, ruler'.

bijenč 'wedding'

-nin: tolturg்un jedi künlerin bu b. da berirbiz saja dag்yn ošolbunuda qulluq üčün (Gen 29:27); tügelledi jedi künlerin bu b. da berdi anar ošol Raḥelni qyzyn anar qatynlyqqa (Gen 29:28)

e Mod.NWKar. bijańć 'joy', Mod.SWKar. bijenc ‘1. joy; 2. wedding', see Németh (2011b: 271).

bikur 'firstfruit'

-larnyn: ki nečik juvutsaj tirkisin b. Adonajg̉a kolos ütülǵan otta jyrtqan kurpa juvutqun ošol tirkisin bikurlaryjnyn (Lev 2:14)

-laryjnyn: ki nečik juvutsaj tirkisin bikurlarnyn Adonajga kolos ütülǵan otta jyrtqan kurpa juvutqun ošol tirkisin b. (Lev 2:14)

-laryn: b. barnerśaĺarnin ki jerlarind́a ki keltirśalar Adonajġa saja bolsun (Num 18:13)

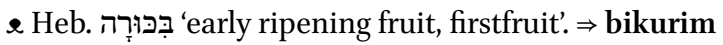




\section{bikurim 'firstfruit'}

olturušlaryjyzdan keltirijiz ötḿagin tenufanyn ekini eki 'esronlardan öźak bolsunlar hamec biširilsinlar b. Adonajġa ki oldu jetilmáagi budajlarny (Lev 23:17); ol b. kününd́a juvutqanyjyzda janġy tirki Adonajg் (Num 28:26); alġyšlaġyn e Adonaj malyn anyn ki oldu ol tunġučlar da ončalar da b. da išin qollarynyn qabul etken ki oldur ol qarbanlar (Deu 33:11)

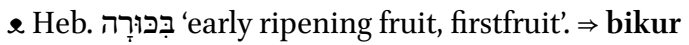

bleha 'metal sheet'

-larny: juġarttylar ošol ol altyn b. (Exo 39:3)

e Ukr. блаха бляха 'metal sheet', Pol. blacha id. $\Rightarrow$ blaḥa

blaḥa 'metal sheet'

-lar: ošol mačmarlaryn ol jazyqlylarnyn ošpularnyn žanlarybyla da qylsynlar alardan juġa b. qaplav mizbeahqqa (Num 17:3)

e Ukr. блаха бляха 'metal sheet', Pol. blacha id. $\Rightarrow$ bleḥa

bohaq 'a kind of skin-disease'

muna terisind́a guflarynyn tunuq temgillar aq symanlylar b. ünd́áladoġan hastalyqty ol jajyldy gufta aruvdu ol (Lev 13:39)

\& Heb. בּה 'a kind of disease', see Klein (1987: 65), 'harmless eruption of the skin, skin-disease', see Koehler \& Baumgartner (1985: 111).

boj: boj qyz 'virgin'

ol qyz jaḩ̌y körümlü edi astry b. q. da kiši bilmedi any (Gen 24:16); tuvdug்u üčün ol b. q. üčün ol juvuqraq anar (Lev 21:3); ki bolsa b. q. kelešingen kišige da tapsa any kiši šaharda da jatsa birgesine (Deu 22:23)

-larnyn: eger klemese atasy ol qyznyn berme any anar qatynlyqqa kümüš ölčesin qalynykibik ol b.q. (Exo 22:16)

-ny: ki jeldese kiši b. q. ki kelešinmegenni da jatsa birgesine qalynlama qalynlasyn any özüne qatynlyqqa (Exo 22:15); tulnu da sürülǵanni erind́an da jengil bašlyny zonany ošol bularny almasyn ki ančaq b. q. uluslaryndan özünün alsyn qatyn (Lev 21:14); ki tapsa kiši b. q. ki kelešinmedi da tutsa any dajatsa birgesine da tabulsalar (Deu 22:28); tyšqartyn tuv eter qylyč da ḥǔ̌uralardan qorquv dag்yn jigitni dag்yn b. q. ömedog்an ulanny bir kišibyla (Deu 32:25)

-y: ̌̌urum alsynlar andan jüz kümüš da bersinler atasyna ol qyznyn ki čygardy jaman slava b. q. üstüne Jisra’elnin (Deu 22:19)

e Mod.NWKar. boj '1. separate; 2. free', Mod.SWKar. bojluk 'virginity'. $\Rightarrow$ bojluqlar

bojavly 'colourful'

ošol ol b. upraqlarny (Exo 31:10); ošol ol b. upraqlarny jumuš etḿa qodešte (Exo 35:19); ol kökten da ol jipkinden da ol qyrmyzy jipekten qyldylar b. upraqlar (Exo 39:1); ošol ol b. upraqlarny (Exo 39:41)

e Mod.NWKar. bojav 'paint' + deriv. suff.

bojluqlar 'virginity'

qojsa anar syltav sözler da čygarsa anyn üstüne jaman slava da ajtsa ošol ol qatynny 
ošpunu aldym da juvudum anar da tapmadym anar b. (Deu 22:14); muna ol qojdu anar syltav sözleri ajtadoġač tapmadym qyzyja b. da bulardylar bojluqlary qyzymnyn da jajsynlar ol juvurganny alnynda qartlarynyn ol šaharnyn (Deu 22:17); eger kerti bolsa ol nerse ošpu tabulmasalar b. qyż்a (Deu 22:20)

-y: da ol kohen gadol qatynny b. byla alsyn (Lev 21:13); muna ol qojdu anar syltav sözleri ajtadogač tapmadym qyzyja bojluqlar da bulardylar b. qyzymnyn da jajsynlar ol juvurġanny alnynda qartlarynyn ol šaharnyn (Deu 22:17)

-yn: alsyn atasy ol qyznyn da anasy anyn da čyg்arsynlar ošol b. ol qyznyn qartlaryna ol šaharnyn ol töre üvge (Deu 22:15)

e Mod.SWKar. bojluk 'virginity' + pl. suff. $\Rightarrow$ boj: boj qyz

bojvol 'buffalo'

-nu: soġag்ny da jürnü da b. (Deu 14:5)

e Russ. буйвол 'bufallo', Mod.NWKar. bujvol 'buffalo'.

bog்uz '(sea) shore'

-unda: da b. ol tengiznin (Deu 1:7)

e Mod.NWKar. bohoz '1. haven, harbour; 2. shore'.

boš: boš etli 'thin'

-ler: jedi tanalarözgelar čyǵadylar alar artyna ol Nild́an jaman körümlüler da b. e. da turdular janynda ol tanalarnyn (Gen 41:3)

e Mod.NWKar. boš 'empty', Mod.NWKar. et 'flesh, meat' + deriv. suff.

bozov 'calf'

alg̀nn maja üč b. da üč ečki da üč qočqar (Gen 15:9)

¿ Mod.NWKar. buzov 'calf'.

böbej 'pupil (of an eye)'

-in: abrady any micvalarybyla abraġanlaj kiši b. közünün (Deu 32:10)

e Mod.NWKar. beb́aj 'pupil (of an eye)', Mod.SWKar. bebej id.

bödev 'barren'

da edi Saraj b. joġedi anar tuvmuš (Gen 11:3o); bolmasty tuv etüvčü da b. qatyn jerijde ošol senin künlerïnin tügellermen (Exo 23:26)

^ Mod.NWKar. bedá 'barren'. $\Rightarrow$ böd́av

böd́av 'barren'

tefile etti Jichaq qatyny üčün ki b. edi ol da qabul etti tefilesin anyn Adonaj da hamila boldu Riveqa qatyny anyn (Gen 25:21); kördü Adonaj ki horlanġandy Le’a da ačty ošol qursag்yn anyn da Raḥel edi b. (Gen 29:31); alġyšlyraq bolursen sen bar ol uluslardan bolmasty send́a b. erkiši da b. qatyn da tuvaryjdada (Deu 7:14)

e Mod.NWKar. bedáv 'barren'. $\Rightarrow$ bödev

bölör 'crystal'

-ü: išikibik kirpičnin išlengen [b]. byla safir tašynyn (Exo 24:10)

e Mod.EKar. bilör 'crystal'.

budaj: qara budaj *'rye'

da ol budaj da ol q. b. vatylmadylar ki jabuq edilar alar (Exo 9:32) 
\& Tkc. qara bugdaj 'rye' and its phonetic variants widespread in Kipchak Turkic, see Stachowski (2008: 79-80).

but 'shaft'

-u:qylg̈yn čyraqba aruv altyndan qaqqan qylynsyn ol čyraqba b. anyn da qamušu anyn (Exo 25:31)

-un: aruv altyndan qaqqan qyldy ošol ol čyraqbany b. anyn da qamušun anyn (Exo 37:17)

-una: budu iši ol čyraqbanyn qaqqan altyndan b. dejin japrajgnadejin (Num 8:4)

\& Mod.NWKar. but 'hip'.

buvgalaq 'groats'

kesildi tamčysy ol čyqnyn da muna jüzleri üstüne ol midbarnyn uvaq b. uvaq moroz kibik ol jer üstüne (Exo 16:14)

e Mod.SWKar. buvhalak 'groats'.

buzbetli 'spotted'

-ĺar: kördüm tüš ašyra da muna ol tegeler ol baruvčular ol qojbyla čybarlar bürtüklülar da b. (Gen 31:10); körgün bar ol tegeler ol baruvčular ol qoj byla čybarlar bürtüklülar da b. (Gen 31:12)

^ Mod.EKar. buzbatly 'spotted'.

bükrü 'hunchbacked'

jemeśa b. jemeśa uvaq jemeśa belma közünd́a jemeśa qyršang்y jemeśa qyng்yrajg்an jemeśa šiškan ajipli (Lev 21:20)

\& Mod.SWKar. bikri 'hunchbacked'.

bürkün- 'to get purified'

-dülar: b. ol Levililar da juvdular upraqlaryn (Num 8:21)

-ḿaśa: ol jedinči künd́a arynyr da eger b. ol üčüčü künd́a da ol jedinči künd́a arynmasty (Num 19:12); bar ol tijüvčü ölüǵa žanyna ol adamnyn kiölśa da b. ošol miškanyn Adonajnyn murdar etti (Num 19:13); kiši ki murdar bolsa da b. da eksilir ol žan ortasyndan ol qahalnyn (Num 19:20)

-sün: ol b. anyn byla ol üčünčü künd́a (Num 19:12)

-üjüz: siz toḥtajyz tyšqartyn avulġa jedi künlar bar ol öltürüvčü ร̌anny da bar tijüvčü qyranġa b. ol üčünčü künd́a da ol jedinči künd́a siz da jesirijiz (Num 31:19); bar upraqbyla da bar terili savutbyla da bar išibyla ečki jununun da bar aġačly savut byla b. (Num 31:20)

-ür: bürkkün alar üstüńa b. suvlar (Num 8:7); bar ol tijüvčü ölüǵa žanyna ol adamnyn ki ölśa da bürkünḿaśa ošol miškanyn Adonajnyn murdar etti da eksilir ol žan Jisra’elnin ki b. suvlary bürkülḿadi anyn üstüna murdar bolsun hanuz murdarlyg்y anyn andady (Num 19:13); ošol miqdašyn Adonajnyn murdar etti b. suvlary bürkülḿadi anyn üstüna murdardy ol (Num 19:20); bolsun alarġa ömürlük resimǵa da bürküvčü ol b. suvlaryn juvsun upraq laryn da ol tijüvčüol b. suvlarġa murdar bolsun ol ingirǵadejin 
(Num 19:21); bar nerśa ki keladi otqa ašyryjyz ot ašyra da arynsyn tek b. suvlary byla bürkülsün (Num 31:23)

^ Mod.SWKar. birkin- '1. to get purified; 2 to get sprinkled'.

\section{bürčümek 'lentil'}

-ĺarin: da Ja'aqov berdi 'Esavġa ötmek da ašyn b. da ašady da ičti da turdu da bardy da hor etti 'Esav ošol ol tunġučluqnu (Gen 25:34)

e Mod.NWKar. burž́umak 'lentil'.

bürk- 'to cleanse'

-ma: alsyn b. ošol ol üvnü eki qušlar da erez ag்ačy da qyrmyzy jiṕak da čabor (Lev 14:49)

-sün: b. ošol ol üvnü qanybyla ol qušnun da ol tiri suvlar byla da ol tiri qušbyla da erez ag̉ačy byla da čabor byla da qyrmyzy ol jiṕak byla (Lev 14:52)

e Mod.NWKar. burk- 'to sprinkle'.

\section{bürünče|k 'veil'}

ketirdi Tamar tulluq upraqlaryn üstünd́an da jabundu b. byla (Gen 38:14)

-|gi: turdu Tamar da bardy da keterdi b. üstünd́an (Gen 38:19)

-ni: da aldy Riveqa ol b. da jabundu (Gen 24:65)

e Mod.NWKar. buruńćak 'coverlet, bedspread'.

bütüv 'bruise'

ulannyöltürürmen b. de jarambyla (Gen 4:23); küvükjara küvükjara ornuna ačyqjara ačyq jara ornuna b. jara b. jara ornuna (Exo 21:25)

e Mod.NWKar. bitov 'bruise'.

byla: šemi byla 'in someone's name'

alġyšlama Jisra’elni š. b. Adonajnyn (Deu 21:5)

-j: ol vahttan ki keldim par'oġa sözleme š. b. jaman etti par'o ošpu ulusqa (Exo 5:23)

e Heb. שِ ‘name', Mod.NWKar. byla 'postp. with'.

C

cadiq 'just, righteous'

da bolġajdy nečik c. alaj raša hašša bolġaj saja tör[e]čisimo bar ol jernin (Gen 18: 25)

-lar: šeme bardy enli c. ortasynda ol šaharnyn (Gen 18:24); da ajtty Adonaj eger tapsam Sedomda enli c. ortasynda ol šaharnyn (Gen 18:26); šeme tabulurlar anda on c. da ajtty Tenri čejpamanmen ol onüčünd́a (Gen 18:32)

-lardan: šeme eksilirlar enli ol c. beš čejparmosen beš üčün ošol bar ol šaharny (Gen 18:28)

-larnyn: ham tasetermosen da bošatmasmosen jazyg்yn orunnun enli ol c. zehutu üčün ki ortasynda anyn (Gen 18:24)

-ni: da juvudu Avraham da ajtty ham tasetermosen c. raša byla (Gen 18:23); hašša bol- 
ġaj saja qylmaqtan ošpu iš kibik öltürḿa c. rašabyla (Gen 18:25); eksitmek üčün ol c. ol raša byla (Deu 29:18)

\& Heb. צִ 'just, righteous'.

cara'at 'leprosy'

eger jajylma jajylsa ol c. terida da qaplasa ol c. ošol bar terisin ol hastalyqnyn (Lev 13:12); baqsyn any ol kohen da muna qaplady ol c. ošol bar gufun (Lev 13:3); murdar etsin any ol kohen neteqti ol c. (Lev 13:30); ki bolsa artynda bašynyn jemeśa alnynda bašynyn aq hastalyq qyzyl syman c. jajyladogiandy ol artynda bašynyn jemeśa alnynda bašynyn (Lev 13:42); terid́a barysysajyn qylyndy ol teri iška c. tavusadogian ol hastalyq murdardy ol (Lev 13:51); passim

-nyn: da bolsa terisind́a gufunun ḥastalyg்yna c. (Lev 13:2); körümü ol hastalyqnyn teŕanŕakti ol terisind́an gufunun hastalyg்ydy c. ol (Lev 13:3); hastalyg்y c. ki bolsa adamda keltirilsin ol kohenǵa (Lev 13:9); hastalyġydy c. ol čyban byla jajyldy (Lev 13:20); murdar etsin any ol kohen hastalyg்ydy c. ol (Lev 13:25); passim

-tan: bujursun ol kohen da alsynlar c. arynuvču kišiǵa eki polnyj qušlar (Lev 14:4); bürksün ol kohen ol arynuvču kiši üstüńa ol c. jedi keŕatlar (Lev 14:7)

-ty: qartajgan c. ol terisind́a gufunun da murdar etsin any (Lev 13:11); baqsyn ol kohen ošol čij etni da murdar etsin any ol čij et murdardy ol c. ol (Lev 13:15); teŕanŕakti ol teridan c. ol (Lev 13:25); c. ol otta küvdürülsün (Lev 13:52); c. ol otta küvdürgün any (Lev 13:57); passim

-tyr: körsün ol kohen da muna jajyldy ol mispahat terid́a da murdar etsin any ol kohen c. ol (Lev 13:8)

-yna: c. ol upraqnyn da üvnün (Lev 14:55)

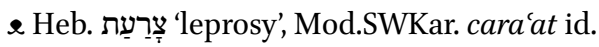

cara'atly 'leprous'

kijirgin qolujnu qojnuja da kijirdi qolun qojnuna da čygardy any da muna boldu qolu anyn c. qarkibik (Exo 4:6)

-ny: bujurgiun ulanlaryna Jisra’elnin da sürsünlar ol Avuldan bar c. da bar zavlyny da bar murdarny žanüčün (Num 5:2)

e Heb. צִרַעַת 'leprosy' + deriv. suff.

cav 'a kind of a lizard; great lizard (?), thorn-tailed lizard (?)'

budur sizǵa ol murdar qumuz da ol tebŕańadogian ol jer üstüna ol holed da ol 'ahbar da ol c. 3̌ynsysajyn (Lev 11:29)

\& Heb. צָז 'a kind of a lizard', see Klein (1987:539), 'thorn-tailed lizard', see Koehler \& Baumgartner (1985: 790).

cicit 'tassel'

sözlagin ulanlaryna Jisra’elnin da ajtqyn alarġa da qylsynlar özlarińa c. et́aklari üstüna upraqlarynyn dorlarysajyn da bersinlar c. qatyna ol etaknin kök šnur (Num 15:38)

e Heb. צִיצִת 'tassel'. 
cyn 'tin'

-ny: tek ošol ol altynny da ošol ol kümüšnü da ošol ol tučnu ošol ol temirni ošol ol c. da ošol ol qorgašynny (Num 31:22)

\& Pol. cyna 'tin'.

cynamon 'cinnamon'

c. ijisli jarymyn anyn (Exo 30:23)

\& Pol. cynamon 'cinnamon'.

$\check{\mathrm{C}}$

čerepli 'earthen, clay'

č. savut ki biširilśa anyn ičińa (Lev 6:21); bar č. savut (Lev 11:33); bujursun ol kohen da sojsun ošol ol qušnu ol birisin č. savutqa tiri suvlar üstüńa (Lev 14:5); alsyn ol kohen aziz suvlar č. savutqa (Num 5:17)

e Ukr. чepen 'erthen pot' + Kar. deriv. suff., Mod.SWKar. cerepli 'earthen, clay'.

čerüv '1. war; battle; 2. military service'

ešitti Johošua' ošol avazyn ol ulusnun qyčqyradog்anny da ajtty Mošege č. avazy avuldan (Exo 32:17); čuqčušmaġyn alarbyla č. (Deu 2:9); da ošol jerin anyn bašlag்yn tasetmía da čuqčušqun anyn byla č. (Deu 2:24)

-de: qajtsyn üvüne mag்at öler č. da özge kiši qutlar any (Deu 20:5); qajtsyn üvüne mag்at öler č. da özge kiši alajoqqa čyg்aryr any (Deu 20:6); mag்at öler č. da özge kiši alyr any (Deu 20:7)

-ge: ki čyqsaj č. dušmanlaryj üstüne (Deu 20:1); bolġaj juvuġanyjyzdačoq ol č. da juvusun ol kohen da sözlesin ol ulusqa (Deu 20:2); ajtsyn alarġa tynlaġyn e Jisra’el siz juvujsiz bügün č. dušmanlaryjyz üstüne (Deu 20:3); ki čyqsaj č. dušmanlaryj üstüne da berse any Adonaj Tenrij qoluja da jesir etsej jesirin anyn (Deu 21:10); ki alsa kiši janġy qatyn čyqmasyn č. (Deu 24:5); passim $\Rightarrow$-ǵa

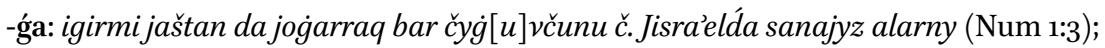
igirmi jaštan da joġarraq bar čyg்vču č. (Num 1:20); bar erkak igirmi jaštan da joġarraq bar čyg்uvč č. (Num 1:22); igirmi jaštan da joġarraq bar čyg்uču č. (Num 1:24); igirmi jaštan da jog்arraq bar čyg்uvču č. (Num 1:26); da čyqty 'Oj biji ol Bašannyn uturularyna alarnyn ol da bar ulusu anyn č. Edre iǵa (Num 21:33); passim $\Rightarrow$-ge -larinin: ajtylady seferind́a č. Adonajnyn (Num 21:14)

-nü: mag்at fašman eter ol ulus körügenlerind́a č. da qajtyrlar Micriǵa (Exo 13:17); ajyrġyn jarymg்a ošol ol halqny arasyna tutuvčularnyn ol č. ol čyg்uvčularnyn čerüvǵa da arasyna bar ol క̌ymatnyn (Num 31:27)

-nün: bolgaj ki učrasalar učurlary č. da artar dag்yn olda dušmanlarymyz üstüne da urušur biznin byla da čyg்ar ol jerd́an (Exo 1:10)

e Mod.NWKar. ćeriv́ '1. army, troops; 2. war; battle' ćerüv 'army, troops', cf. BHeb. צִבְָ '1. military service; 2. host, fighting men'. $\Rightarrow$ čerüv qyl- 
čerüv qyl- 'to make war'

-dy: qondarg̉yn beklik ol šahar qatyna ki ol q. birgeje č. engeninedejin (Deu 20:20)

-dylar: q. č. Ber'a byla bijibyla Sedomnun (Gen 14:2 [inversion])

-sa: eger bazlašmasa birgeje da q. birgeje č. da qysyqlyq etkin anar (Deu 20:12)

e Mod.NWKar. čeriv ‘' 1. army, troops; 2. war; battle' čerüv́ 'army, troops', Mod.NWKar. $k y l$ - 'to do'. $\Rightarrow$ čerüv

češindirt- (dat.) 'to order to take off (garments)'

-kin: č. Aharonġa ošol upraqlaryn da kijdirgin alarny El'azarg்a (Num 20:26)

-ti: č. Moše Aharonġa ošol upraqlaryn anyn da kijdirdi alarny El'azarg்a (Num 20:28) -tilar: nečik keldi Josef qaryndašlaryna da č. Josefke ošol kölmegin ošol ol ojuvlu kölmekni (Gen 37:23)

e Mod.NWKar. češińdir- 'to order to take off (garments)'; ćešińdirt́- contains a doubled causative suffix.

češka 'joint'

-sy: kördü kijenelmedi any da tijdi češkasyna butunun da qoż̇aldy č. butunun Ja'aqovnun čengeškenind́a birgesina (Gen 32:26); ašamajdylar ulanlary Jisra’elnin ošol gid hanašeni ki č. üstüńa ol butnun (Gen 32:33)

-syna: kördü ki jenelmedi any da tïdi č. butunun da qozġaldy češkasy butunun Ja'aqovnun čengeškenind́a birgesina (Gen 32:26); ki tijdi malah č. butnun Ja'aqovnun gid hanašeǵa (Gen 32:33)

e Mod.SWKar. ceska 'joint'.

čirkille- 'to harvest all or most of the fruits'

-megin: -borlalyg்yjny č. özüjartyna ġaripge öksüzge da tulġa bolsun (Deu 24:21)

e Mod.NWKar. čirkiń čirkińlar 'fruit remains after harvest'. $\Rightarrow$ čirkilla-

čirkilla- 'to harvest all or most of the fruits'

-magin: borlalygjyjny č. (Lev 19:10)

e Mod.NWKar. čirkiń čirkińlar 'fruit remains after harvest', cf. cirkinle- in H (Lev 19:10). $\Rightarrow$ čirkille-

čöplöv 'gleaning, gathering'

-ün: orǵanyjyzda ormag்y jerijiznin tüǵallaḿagin qyryjyn tüzüjnün orma da č. ormaġyjnyn čöplaḿagin (Lev 19:9); orġanyjyzda ošolormag்ynjerijiznin tüǵallaḿagin qyryjyn tüzüjnün orġanyjda da č. ormag்yjnyn čöplaḿagin miskinǵa da ġaripǵa kemiškin alarny menmen Adonaj Tenrijiz (Lev 23:22)

e Mod.NWKar. ćopla- 'to gather, to collect' + deriv. suff.

\section{čubrajğan *'stunted'}

-ny: da ögüznü da qojnu artyġač buvunlunu da č. క̌omartlyq qylg̀yn any da nijetka qabul bolunmasty (Lev 22:23)

\& Mod.SWKar. cubraj- '1. to wrinkle, to frown; 2. to wither'.

čuqčuš- '1. to get excited; 2. to get ready, to ready yourself'

-mag̀yn: č. alarbyla čerüv ki berḿanmen saja jerind́an anyn méraslik (Deu 2:9); 
qysyqlyq etŕagin alarġa da č. alarbyla ki bermanmen jerind́an ulanlarynyn 'Amonnun saja méraslik (Deu 2:19)

-majyz: č. alarbyla bermánmen sizǵa jerlarind́an alarnyn (Deu 2:5)

-qun: č. anyn byla čerüv (Deu 2:24)

e Mod.SWKar. cukcus- '1. to get excited; 2. to ready'.

čyġar- 'to harvest'

-mady: da qajsy ol kiši ki ornatty borlalyq da alajoqqa č. any barsyn da qajtsyn üvüne mag்at öler čerüvde da özge kiši alajoqqa čyġaryr any (Deu 20:6)

-massen: qatyn kelešsej da özge kiši alyr any üv qondarsaj da olturmassen anda borlalyq ornatsaj da alajoqqa č. any (Deu 28:30)

-yr: da qajsy ol kiši ki ornatty borlalyq da alajoqqa čagjyrmady any barsyn da qajtsyn üvüne maġa öler čerüvde da özge kiši alajoqqa č. any (Deu 20:6)

\& Mod.NWKar. čyhar- 'to take out'.

čylčal 'a species of locust or cricket'

bar ag்ačyjny da jemišin jerijnin taseter č. byla ündeledogan qurt (Deu 28:42)

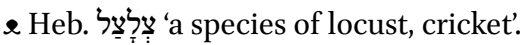

čynyq- *'to train'

-qan: da jasandyrdy ošol č. elin tuvg்anlaryn üvünün (Gen 14:14)

e Mod.NWKar. čynyḥ- 'to get used to'.

čyq: čyġa da qajta 'to and fro'

ijdi ošol ol [qarg்any] da čyqtyč. d. q. quruġunča ol suvlar ol [jer ü]stüńa (Gen 8:7)

e Mod.NWKar. čy h- 'to go out', Mod.NWKar. kajt- 'to return'.

čyq: utru|suna čy $\mid \mathbf{q}-\sim$ uturu|suna čy $\mid \mathbf{q}$ - 'to go out to meet'

-|ġar: da dag்yn muna č. uturuja da körer seni da bijenir kölnünde (Exo 4:14)

-|ġarmen: ajtty anar Edom ašmassen jerimašyra mag்at qylyč byla č. utruja (Num 20:18)

-ty: č. biji Sedomnun uturusuna anyn qajtqanyn dan sortun (Gen 14:17); keldi Ja'aqov ol tüzd́an ingird́a da č. Le’a uturusuna (Gen 3o:16); č. Moše uturusuna qajnatasynyn da bašurdu (Exo 18:7); da jyštyrdy Siḥon ošol bar ulusun özünün da č. uturusuna Jisra'elnin ol midbarg்a (Num 21:23); da č. 'Og் biji ol Bašannyn uturularyna alarnyn ol da bar ulusu anyn (Num 21:33); keladi Bil'am da č. uturusuna anyn (Num 22:36); da č. Sihnon biji Hešbonnun da 'Og் biji ol Bašannyn uturumuż̇a da qyrdyq alarny (Deu 29:6); passim

e Mod.NWKar. čy h- 'to go out', Mod.NWKar. uturu utru 'opposite’.

D

da'a 'a bird of prey; the red kite (?)'

-ny: ošol ol d. da ošol ol ajany žynsysajyn (Lev 11:14)

\& Heb. דָדָ 'a bird of prey, probably the red kite', see Klein (1987: 112), Koehler \&

Baumgartner (1985: 198). $\Rightarrow$ ra’a 
daja 'a bird of prey, probably the kite'

-ny: ol ra’any da ošol ol ajany da ol d. ̌̌ynsysajyn (Deu 14:13)

\& Heb. 꾸 'a bird of prey, probably the kite', see Klein (1987: 121), 'undefinable, forbidden bird', see Koehler \& Baumgartner (1985: 208).

dijament 'diamond'

ol ekinči jerge sürne tašy safir tašy da d. (Exo 28:18); ol ekinči jerge sürne tašy safir tašy dad. (Exo 39:11)

\& Pol. diament 'diamond'.

dišon 'antelope'

-nu: soġagny da jürnü da bojvolnu da pole qojun da d. (Deu 14:5)

e Heb. דִ 'antelope, addax'.

duhifat 'hoopoe'

-ny: ošol ol hasidany ol anafany žynsysajyn da ošol d. da ošol ol jary qanatyn (Lev 11:19); ol hasidany da ol anafany žynsysajyn da ol d. da ol jary qanatyn (Deu 14:18)

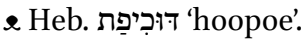

dunja 'eternity, everlastingness'

-ga: mag்at sunar qolun da alyr dag்yn ag்ačyndan ol tirliknin da ašar da tiri bolur d. dejin (Gen 3:22); ošol bar ol jerni ki sen köresen saja berirmen any da urluġuja $d$. dejin (Gen 13:15); budur atym menim d. dejin da budur saġynčym menim har bir dorġa (Exo 3:15); da saqlajyz ošol ol söznü ošpunu resimǵa saja da ulanlaryja d. dejin (Exo 12:24); bügün arttyrmassiz körḿa any d. dejin (Exo 14:13); Adonaj bijlik eter d. dejin da ömürgedejin (Exo 15:18); passim

-nyn: bolur ol jaja bulutta da bajarmen any saġynma šertin d. arasyna Tenrinin da arasyna bar tiri ̌̌annyn (Gen 9:16); berirmen saja da urlug்uja özüjdan sortun ošol tirilmüš jerlerijni ošol bar jerin Kena'annyn tutuvlug்una d. (Gen 17:8); da čag்yrdy anda aty byla Adonajnyn Tenrisinin d. (Gen 21:33); da berirmen ošololjerni ošpunu urlugiuja özüjden sortun tutuvlug்una d. (Gen 48:4); alġyšlary atajnyn küčejdilar alg̀yšlary üstüne tuvduruvčularymnyn belgisinedejin qajalarynyn d. (Gen 49:26); da bašyndan avaldaġy tavlarnyn da nametinden qajalarynyn d. (Deu 33:15); passim

^ Mod.NWKar. dunja '1. world; 2. people', cf. Heb. עוֹ '1. eternity; 2. world'.

dušman: dušman bol- $(a b l$. $)$ 'to be hostile to somebody'

-du: d. b. 'Esav Ja'aqovg்a ol alġyš üčün (Gen 27:41)

-dular: kördülar qaryndašlary anyn ki süvdü atasy alarnyn jahšyraq bar qaryndašlaryndan anyn da d. b. anar da bolalmadylar sözlemia anar bazlyqbyla (Gen 37:4); ačy ettilar anar da attylar any d. b. anar oq jesileri (Gen 49:23)

-ma: da arttyrdylar daġyn d. b. anar (Gen 37:5); arttyrdylar d. b. anar tüšlari üčün da sözlari üčün anyn (Gen 37:8)

-mag்yn: d. b. qaryndašyja (Lev 19:17)

-sam: eger men d. b. saja ki ašmaġajmen saja ošol ol obany (Gen 31:52)

-saj: eger sen d. b. maja ki ašmaġajsen maja ošol ol obany (Gen 31:52) 
-ur: da ajttylar šeme d. b. bizǵa Josef da qajtarma qajtaryr bizǵa ošol bar ol jamanlyqny ki töledik anar (Gen 50:15)

-urmen: $d$. b. dušmanlaryja da qysyqlyq etermen qysyqlyq etüvčülerije (Exo 23:22)

e Mod.NWKar. dušman 'enemy' + aux. verb.

క̌omartlat- 'to make generous'

-sa: har kišiden ki ̌̆. any jüregi anyn (Exo 25:2)

-ty: kim ki ̌̌. žany anyn (Exo 35:21)

e Mod.NWKar. ̌̌omart 'generous' + deriv. suff.

žomartlyq 'freewill offering'

bar kiši da qatyn ki žomartlatty jüregi alarnyn alarny keltirme bar ol iške ki bujurdu Adonaj qylma naviligi ašyra Mošenin keltirdiler ulanlary Jisra’elnin ž. Adonajġa (Exo 35:29); aldylar alnyndan Mošenin ošol bar ol terumany ki keltirdiler ulanlary Jisra’elnin išine ol aziz qulluqnun qylma any da ulanlary Jisra'elnin keltirdiler anar dag்yn گ̆. har ertenbylada (Exo 36:3); eger nijet jemeśa క̌. bolsa debehasy qarbanynyn juvutqan künd́a ošol debehasyn ašalsyn da tanbyladanda ol qalġan andan ašalsyn (Lev 7:16); ögüznü da qojnu artyg்ač buvunlunu da čubrajganny ž. qylgyn any da nijetka qabul bolunmasty (Lev 22:23); kinečiknijet ettij Adonajġa Tenrije ž. ki sözledijavzujbyla (Deu 23:24)

-lary: ki juvutsa qarbanyn özünün bar nijetĺarisajyn da bar ̌̆. sajyn alarnyn ki juvutsalar Adonajga (Lev 22:18)

-laryjny: bolalmaġyn ašama šaharlaryjda ončasyn bürtügüjnün da šarbetijnin da sajyjnyn da tung்učlaryn syg்yryjnyn da qojujnun da bar nijetlerijni ki nijet etsej da ̌̆. da terumasyn qolujnun (Deu 12:17)

-laryjyzdan: bašqa barnijetlarijizd́an da bašqa bar ̌̌. ki berśajiz Adonajġa (Lev 23:38); bulardylar ol musaflary ki qylyjyz Adonajga mo'edĺarijizd́a bašqa nijetlarijizd́an da ̌̌. 'olalaryjyzsajyn da tirkilarijizsajyn da nesehĺarijizsajyn da šelamimlarijizsajyn (Num 29:39)

-laryjyzny: da keltirijiz ary ólalaryjyzny da debehalaryjyzny da ošol ončalaryjyzny da ošol terumasyn qolujuznun da nijetlerijizni da ̌̌. da tung்učlaryn sygjyryjyznyn da qojujuznun (Deu 12:6)

e Mod.NWKar. ̌̌omartlyh 'generosity'.

E, ' $\mathrm{E}$

efa 'ephah (a grain measure)'

rast e. bolsun bürtük ölćaḿakka (Lev 19:36)

-nyn: ol 'omer onunču ülüšü ol e. edi ol (Exo 16:36); onunču ülüšü ol e. özek jumurul- 
ġan jančqan jav byla (Exo 29:40); onunču ülüšün ol e. öźak hatatqa (Lev 5:11); onunču ülüšü ol e. öźak tirki (Lev 6:13); onunču ülüšün ol efanyn unun arpalarnyn (Num 5:15); passim

\& Heb. אֵיפָה 'ephah, a Hebrew grain measure’.

efod 'ephod'

bulardylar ol upraqlar ki qylsynlar ḥošen da e. da qaftan da ojuvlu kölmek (Exo 28:4); kötürülmesin ol hošen ol e. üstünden (Exo 28:28); kötürülmegej ol hošen ol e. üstünden (Exo 39:21)

-g்a: šoham tašlary e. (Exo 25:7); šoham tašlary e. da milu’im tašlary ḥošenge (Exo 35:9); ol nasiler keltirdiler ošol ol šoham tašlaryn e. da ošol ol milu’im tašlaryn hošenge (Exo 35:27)

-nu: qylsynlar ošol e. altyndan kökten da jipkinden da qyrmyzy jipekten da ešken bezden iši ojuvlavčunun (Exo 28:6); qyldy ošol ol e. altyndan kökten da jipkinden da qyrmyzy jipekten da ešken bezden (Exo 39:1); berdi anyn üstüńa ošol ol e. (Lev 8:7)

-nun: qojg்un ošol eki ol tašlarny javrunlary üstüne ol e. (Exo 28:12); iši kibik e. (Exo 28:15); da bergin javrunlary üstüne ol e. (Exo 28:25); qylg̀yn eki jüzükler altyndan da qojg̀un alarny eki učlary üstüne ol hošennin qyryj üstüne anyn ki janyna ol e. ičkerige (Exo 28:26); qylg̀yn eki jüzükler altyndan da bergin alarny eki javrunlary üstüne ol e. ašaġartyn (Exo 28:27); passim

\& Heb. אي 'ephod'. $\Rightarrow$ efodla-

efodla- 'to put on the ephod (on a person)'

-dy: da kijdirdi anar ošol ol qaftanny da berdi anyn üstüna ošol ol efodnu da bajlandyrdy any ojuvubyla ol efodnun da e. any anyn byla (Lev 8:7)

^ Heb. אی 'ephod' + Kar. deriv. suff. $\Rightarrow$ efod

El Šadaj 'God Almighty'

da aškara boldu šehinam Avrahamġa Jichaqqa da Ja'aqovġa E. Š. byla vale šemim byla Adonaj bilinmedim alarġa (Exo 6:3)

e Heb. אֵל שַָׁדי 'God Almighty'.

elek *'grate, grating'

qylg̀yn anar e. iši avnyn tučtan (Exo 27:4); qyldy mizbeahqa e. iši avnyn tučtan (Exo 38:4)

-ke: qojdu dört jüzükler dört ol učlarda ol tuč e. orunlar tutqučlarg்a (Exo 38:5)

-ni: tuč mizbeahny da ošol ol tuč e. ki anar (Exo 38:30); tuč mizbeaḥny da ošol tuč e. ki anar (Exo 39:39)

¿ Mod.SWKar. elek '1. net; 2. riddle, sieve', Mod.NWKar. elak 'sieve'.

'enajet 'eye (of God)'

-laryjda: ajtty Moše Adonajg̉a nek jaman ettij quluja da nek tapmadym širinlik 'e. (Num 11:11); eger taptym eśa širinlik 'e. da körmájim jamanymny (Num 11:15)

-larynda: da qylsajyz ol jaman körünǵanni e e. Adonajnyn (Deu 4:25); qylg̀yn ol tüz da ol jaḥšy körünǵanni 'e. Adonajnyn (Deu 6:18)

e Mod.NWKar. 'enajat 'eye (of God)'. 
erez 'cedar'

da e. ag்ačy (Lev 14:4); ol tiri qušnu alsyn any da ošol ol e. ag்ačny (Lev 14:6); alsyn bürkḿa ošol ol üvnü eki qušlar da e. ag்ačy da qyrmyzy jiṕak da čabor (Lev 14:49); alsyn ošol ol e. ag்ačyn (Lev 14:51); da e. ag்ačy byla (Lev 14:52)

-ĺarni: öźanlar kibik qajyrylġan dylar baġalar kibik öźan qatyna čandallarny kibik ornatty Adonaj e. kibik suvlar qatyna (Num 24:6)

\& Heb. אֶר 'cedar'.

ertenbyla 'early'

-da: tünlej turdu Avraham e. ol orunġa ki turdu anda alnynda Adonajnyn (Gen 19:27); tünlaj turdu Avraham e. da jerledi ošol ešegin (Gen 22:3); ašadylar da ičtilar ol da ol erenlar ki birgesińa da qondular da turdular e. da ajtty ijijiz meni (Gen 24:54); tünlej turdu Jáaqov e. da aldy ošol ol tašny (Gen 28:18); tünlej turdu Lavan e. da öptü ulanlaryn da qyzlaryn (Gen 32:1); passim

-g̀a: da ošol bar ol artyg்ačny qaldyryjyz özüjüzǵa saqlavg்a ol e. dejin (Exo 16:23); qojdular any ol e. dejin ki nečik bujurdu Moše (Exo 16:24); eger qalsa etinden ol milu'imnin da ol ötmekten ol e. dejin da küvdürgün ošol ol qalġanny otta (Exo 29:34); bolgun hadir e. da mingin ertenbylada tavyna Sinajnyn (Exo 34:2)

-nyn: ošol ol ekinči qozunu qylg̀yn ol eki ingirler arasyna tirkisikibik ol e. (Exo 29:41)

\& Mod.SWKar. ertenbyla 'at dawn, early'.

esirtüvčü 'strong drink'

čag்yr da e. ičmagin sen da uvullaryj birǵasińa (Lev 10:9); qujma neseh e. alnynda Adonajnyn (Num 28:7)

-dan: vinadan da e. ajyrsyn özün sirkasin vinanyn da esirtüvčünün ičmiasin (Num $6: 3)$

-nün: vinadan da esirtüvčüd́an ajyrsyn özün sirkasin vinanyn da e. ičmasin (Num $6: 3)$

e Mod.NWKar. esirtki 'strong drink'.

'esron 'one tenth, tenth part'

-lar: üč e. öźak tirki jumurulgan javbyla da bir log jav (Lev14:10); tirkisi anyn eki é öźak jumurulġan javbyla (Lev 23:13); qočqarg்a qylg̀nn tirki öźak eki 'e. jumurulġan javbyla üčüčü ülüšü ol hinnin (Num 15:6); juvutsun balasy qatyna ol sygyrnyn tirki öźak üč ee. jumurulgan javbyla jarymy ol hinnin (Num 15:9); ol šabat kününd́a eki qozular jyllyq balalary tüǵallarni da eki e. öźak tirki jumurulġanjavbyla da nesehi anyn (Num 28:9); passim

-lardan: keltirijiz ötmáagin tenufanyn ekini eki 'e. öźak (Lev 23:17)

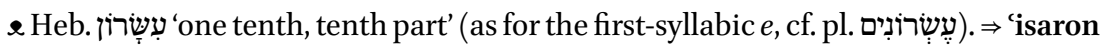
ešek 'female donkey'

-ĺar: teveler ömüzdüredog்anlar balalarybyla otuz tiši tanalar qyrq da erkek tanalar on e. egirmi da qatyrlar on (Gen 32:16)

e Mod.NWKar. ešak 'donkey'. 
ezil- *'to be bruised'

-ǵanni: e. da jančylġanny da üzülǵanni da kesilǵanni juvutmajyz Adonajġa da jerijizd́a qylmajyz (Lev 22:24)

e Mod.EKar. ezil- 'to be creased, to be crumpled', Mod.SWKar. ez- 'to crush; to bruise; to flatten'.

F

farst *'1. (wood-beamed) ceiling; 2. (wood-beamed) wall, side'

-laryn: da qaplag்yn any aruv altyn byla ošol čardag்yn anyn da ošol f. anyn čüvre (Exo 3०:3)

-larynda: qajtsyn ol kohen ol jedinči künd́a da körsün da muna jajyldy ol hastalyqf. ol üvnün (Lev 14:39)

-qa: kördü ol eśak ošol malahyn Adonajnyn da qysyldy olf. (Num 22:24); da qysty ošol ajagyn Bil'amnyn olf. da arttyrdy urma any (Num 22:25)

-tan: da körümlari alarnyn juvuzraqty olf. (Lev 14:37)

-y: da tamyzdyrylsyn qany anyn f. üstüne ol mizbeahnyn (Lev 1:15); bürksün qanyndan ol hatatnyn f. üstüńa ol mizbeahnnyn (Lev 5:9)

-ymnyn: ančaq ošpu erenĺarǵa qylmajyz neḿade anyn üčün qačan ki keldilar syjynma kölegesind́af. (Gen 19:8)

-yndan: sürüvlari ol šaharlarnyn ki berśajiz Levililarǵaf. ol šaharnyn da tyšqaryg்a min lokot čüvŕa (Num 35:4)

^ Pol. arch. forst 'wood-beamed ceiling' (SStp. II 366; de Vincenz \& Hentschel 2010, s.v. forszt I), cf. Heb. קוֹרָה 'rafter, beam' in Gen 19:8.

fartuh 'apron'

-lar: da qyldylar özlerina f. (Gen 3:7)

\& Pol. fartuch 'apron', Ukr. Øapmyx id.

fleška 'bottle'

da aldy ötmek da f. suvda berdi Hag்arg்a (Gen 21:14)

\& Pol. flaszka 'bottle'. $\Rightarrow$ fleške

fleške 'bottle'

-den: da tügendilar ol suvlar olf. (Gen 21:15)

-ni: da bardy da tolturdu ošol ol f. suv da ičirdi ošol ol ulanny (Gen 21:19)

e Pol. flaszka 'bottle'. $\Rightarrow$ fleška

G

galban 'galbanum'

ajtty Adonaj Mošege alġynözüje otjamlar mušk da revent da g. otjamlar da aruv levona bašqa bašqa har birisi bolsun (Exo 30:34)

^ Pol. galban 'galbanum', Russ. гальбан id. 
galgal 'globe, celestial sphere'

-larnyn: Adonaj Tenrijiz oldu Tenrisi ol malahlarnyn da biji ol g. (Gen 10:17)

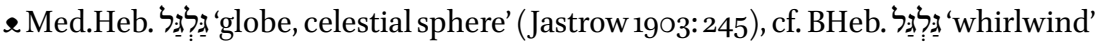
in Psalm 77:18.

gibeah 'bald on the forehead'

eger qyryjyndan jüzlarinin julqunsa bašy anyn g. ünd́áladi ol aruvdu ol (Lev 13:41)

\& Heb. בִבְבר 'bald on the forehead'.

gid hanaše 'sinew of the thigh'

-ǵa: ki tijdi malah̆ češkasyna butnun Ja'aqovnun g. h. (Gen 32:33)

-ni: anyn üčün ašamajdylar ulanlary Jisra’elnin ošol g. h. ki češkasy üstüna ol butnun (Gen 32:33)

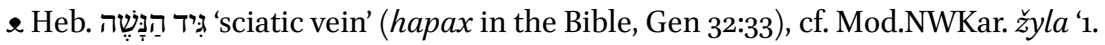
vein; 2. sinew'.

gög *'1. 1/20 of mithqal (a unit of weight); 2 . half a mithqal (a unit of weight)'

aldy ol kiši altyn syrg்a g. ölčövü anyn (Gen 24:22); g. baštan jarymy ol mitqalnyn mitqaly byla ol qodešnin (Exo 38:26); da bar qyjasyj bolsun mitqaly byla ol qodešnin igirmi g. bolsun ol mitqal (Lev 27:25)

-dü: jarymyn ol mitqalnyn mitqaly byla ol qodešnin egirmi g. ol mitqal jarymyn ol mitqalnyn teruma Adonajg்a (Exo 30:13); alġyn bešar bešar mitqallar bašsajyn ol aziz mitqal byla alg̀yn igirmi g. ol mitqal (Num 3:47); alg̀yn qyjasyj byla kümüš beš mitqallar mitqaly byla ol qodešnin igirmig. ol (Num 18:16)

\& Cf. SWKar. geg in H (Gen 24:22).

grunt 'floor'

-unda: alsyn ol kohen aziz suvlar čerepli savutqa da ol topraqtan ki bolsa g. ol miškannyn alsyn ol kohen da bersin ol suvlarġa (Num 5:17)

\& Pol. grund 'ground', Russ. грунт id.

güvün 'cud'

bar tujaqlavčunu tujaq da ajyruvčunu ajyryčyn tujaqlarnyn g. keltirüvčünü tuvardan any ašajyz (Lev 11:3); tek ošol bunu ašamajyz g. keltirüvčülard́an jalġyz ol güvünnü da tujaqlavčulardan jalg̀yz ol tujaqny (Lev 11:4); ošol ol tev́ani hota g. keltiradi eśad́a ol da tujaq tujaqlamajdy murdardy ol sizǵa (Lev 11:4); ošol ol krolikni hota g. keltiradi eśad́a ol da tujaq tujaqlamajdy murdardy ol sizǵa (Lev 11:5); ošol ol qojanny hot́a g. keltiŕadi eśad́a ol da tujaq tujaqlamajdy murdardy ol sizǵa (Lev 11:6); ošol ol hazirni hota tujaqlajdy tujaq ol da ajyrady ajyryčyn tujaqnyn da olg.güvünlaḿajdi murdardyr ol sizǵa (Lev 11:7); bar ol tuvar ki ol tujaqlajdy tujaq da ajyryč ajyrmajdy da g. keltirmajdi murdardylar alar sizǵa (Lev 11:26); bar tuvarny tujaqlavčunu tujaq da ajyruvčunu ajyryčyn eki tujaqlarny g. keltirüvčünü tuvarda any ašajyz (Deu 14:6); ošol ol teveni da ošol qojanny da ošol ol krolikni hote g. keltiredilerde ese alar da tujaq tujaqlamajdylar murdarlardylar alar sizge (Deu 14:7); ošol ol hazirni hote tujaqlajdy esede tujaq ol da g. keltirmejdi murdardy ol sizge (Deu 14:8) 
-nü: tek ošol bunu ašamajyz güvün keltirüvčülard́an jalġyz ol g. da tujaqlavčulardan jalg̀yz ol tujaqny (Lev 11:4); tek ošol bunu ašamajyz jalġyz keltirüvčülerden ol g. da jalġyz tujaqlavčulardan ol tujaqny ol ajyrylganny (Deu 14:7)

e Mod.NWKar. ǵuv́ 'cud', Mod.SWKar. givin 'cud'. $\Rightarrow$ güvün keltir-, güvünĺa-

güvün keltir- 'to regurgitate'

-adi: ošol ol tev́ani hotá g. k. eśad́a ol da tujaq tujaqlamajdy murdardy ol sizǵa (Lev 11:4); ošol ol krolikni ḥot́a g. k. eśad́a ol da tujaq tujaqlamajdy murdardy ol sizǵa (Lev 11:5); ošol ol qojanny hotáa g. k. eśad́a ol da tujaq tujaqlamajdy murdardy ol sizǵa (Lev 11:6)

-diler: ošol ol teveni da ošol qojanny da ošol ol krolikni hote g. k. de ese alar da tujaq tujaqlamajdylar murdarlardylar alar sizge (Deu 14:7)

-mejdi: ošol ol hazirni ḥote tujaqlajdy esede tujaq ol da g. k. murdardy ol sizge (Deu 14:8) $\Rightarrow$-majdi

-majdi: bar ol tuvar ki ol tujaqlajdy tujaq da ajyryč ajyrmajdy da g. k. murdardylar alar sizǵa (Lev 11:26) $\Rightarrow$-mejdi

-üvčülard́an: tek ošol bunu ašamajyz g. k. jalg̀yz ol güvünnü da tujaqlavčulardan jalgyz ol tujaqny (Lev 11:4)

-üvčünü: bar tujaqlavčunu tujaq da ajyruvčunu ajyryčyn tujaqlarnyn g. k. tuvardan any ašajyz (Lev 11:3); bar tuvarny tujaqlavčunu tujaq da ajyruvčunu ajyryčyn eki tujaqlarny g. k. tuvarda any ašajyz (Deu 14:6);

e Mod.NWKar. ǵuv 'cud', Mod.SWKar. givin 'cud', Mod.NWKar. keltir- 'to bring'. $\Rightarrow$ güvün, güvünĺa-

güvünla- 'to chew the cud'

-majdi: ošol ol hazirni ḩot́a tujaqlajdy tujaq ol da ajyrady ajyryčyn tujaqnyn da ol güvün g. murdardyr ol sizǵa (Lev 11:7)

e Mod.NWKar. ǵuvíla- 'to chew the cud', Mod.SWKar. givinle- 'to chew the cud'. $\Rightarrow$ güvün, güvün keltir-

\section{H,}

ḥagav 'grasshopper (?)'

-ny: ošol bunlary alardan ašajyz ošol ol arbeni žynsysajyn da ošol sal'amny žynsysajyn da ošol ol hargolnu žynsysajyn ošol ol ḥ. ̌̌ynsysajyn (Lev 11:22)

\& Heb. ITז 'locust, grasshopper', see Klein (1987: 207), 'a kind of locust allowed for food', see Koehler and Baumgartner (1985: 275).

hajifsin- 'to have mercy'

-mesin: keskin ošol uvučun anyn ḥ. közüj (Deu 25:12)

e Mod.NWKar. hajyfsun- hajyfsyn- 'mercy'. $\Rightarrow$ hajifsinmáak, hajifsün-

hajaifsinma|k 'mercy'

-|gi: da tuttular ol erenlar qolundan anyn da qolundan qatynyn da qolundan eki qyzlarynyn h. byla Adonajnyn anyn üstüńa (Gen 19:16) 
e Mod.NWKar. hajyfsunmaḥ hajyfsynmaḥ 'mercy' $\Rightarrow$ ḥajifsin-, ḥajifsün-.

hajifsün- 'to have mercy'

-dü: ki h. meni Tenri da ki bardy maja baryndan (Gen 32:12); debehasydy qarbanynyn Pesahnnyn ol Adonajga ki h. üvleri üstüne ulanlarynyn Jisra’elnin Micrida (Exo 12:27)

-gej: da ajtty Tenri h. seni uvlum (Gen 43:29) $\Rightarrow$-ǵaj

-gejmen: ki h. da raḥmetlermen ošol kimni ki ušajdy ki raḥmetlegejmen (Exo 33:19)

-ǵaj: Jarytqaj Adonaj qyblalaryn özünün saja da h. seni (Num 6:25) $\Rightarrow$-gej

-mesin: közlerijiz h. savutlaryjyz üstüne (Gen 45:20); da h. közüj anyn üstüne da žallemegin da jašyrmag்yn günehin anyn (Deu 13:9); h. közüj anyn üstüne da eksitkin borčun ol könü qannyn (Deu 19:13); ḥ. közün ̌̌an ̌̌an ornuna (Deu 19:21)

-mesti: ulan üstüne h. (Deu 28:50)

-magin: veŕan etḿa veŕan etkin alarny kesḿagin alar byla šert da h. alarny (Deu 7:2) -masin: da tavusursen ošol bar ol uluslarny ki Adonaj Tenrij beradi saja h. közüj alar üstüńa (Deu 7:16)

-üp: ol ulanlar ki h. berdi Tenri quluja (Gen 33:5)

-ürmen: da körermen ošol ol qanny da h. üstüjüzge (Exo 12:13)

-üvčü: küčlü Tenri raḥemlenüvčü da ḥ. uzaq ačuvlu da köp šavaġatly da kerti (Exo 34:6)

e Mod.NWKar. hajyfsun- hajyfsyn- 'mercy'. $\Rightarrow$ hajajifsin-, ḥajifsinmak

ḥala 'cake'

bohon ötmek bir da h. ötmek javly bir (Exo 29:23); aldy h. maca bir da h. ötḿakjavly bir (Lev 8:26); ekšar isarondan bolsun ol bir h (Lev 24:5); qojg்un any eki tüzüvlar altyšar h. bolsun ol bir tüzüvd́a (Lev 24:6); alsyn ol kohen ošol ol bilakni biškanni ol qočqardan da h. maca bir ol četandán (Num 6:19); passim

-lar: ötmek macalar da h. macalar jumurulganlar javbyla (Exo 29:2); ki juvutsaj qarban tirki biširilǵan pečta öźak h. macalar jumurulganlar javbyla da juġa macalar jaġylganlar javbyla (Lev 2:4); juvutsun debehasy qatyna ol šükürlüknün h. macalar jumurulganlar javbyla (Lev 7:12); öźak qatlanġan h. jumurulġanlar javbyla (Lev 7:12); alġyn öźak da biširgin any on eki h. (Lev 24:5); passim

-lary: h. byla qužur ötḿaknin juvutsun qarbanyn (Lev 7:13)

\& Heb. חַלָ 'a kind of cake, loaf; hallah, i.e. the priest's share of the dough'.

halaf 'slaughtering knife'

-ny: aldy Avraham ošol otunlaryn ol 'olanyn da qojdu Jichaq uvlu üstüna da aldy qoluna ošol ol otnu da ošol ol h. da bardylar eksilerid́a birǵa (Gen 22:6); da sundu Avraham ošol qolun da aldy ošol ol ḥ. sojma ošol uvlun (Gen 22:10)

e Heb. חָָָ 'slaughtering knife'.

hali 'carpet'

-lej: čyqty ol burung்usu qyzyl barčasy ḥ. tüklü da atadylar atyn anyn 'Esav (Gen 25:25)

e Mod.SWKar. hali 'carpet'. 
ḥamec 'leavened (that which is leavened)'

da ašalmasyn h. (Exo 13:3); macalar ašalsyn jedi ol künlerde da körünmesin saja h. da körünmesin saja quگ̌ur bar čegïde (Exo 13:7); debeḥa etmegin ḥ. qatyna (Exo 23:17)

e Hebr. חָמָץ 'that which is leavened'.

hanot et- 'to enbalm'

-me: bujurdu Josef qullaryna özünün ol rofelerge h. e. ošol atasyn (Gen 50:2)

-tiler: h. e. ol rofeler ošol Jisra’elni (Gen 50:2) $\Rightarrow$-tilar

-tiĺar: öldü Josef jüz da on jašar da h. e. any da qojuldu aronġa Micrid́a (Gen 50:26)

$\Rightarrow$-tiler

\& Hebr. חָזָ 'to enbalm', Heb. לָ 'to enbalm' + Kar. aux. verb.

hanot etil- 'to be enbalmed'

-genlernin: toldular anar qyrq kün ki alaj tolaredilar künleri ol h. e. (Gen 50:3)

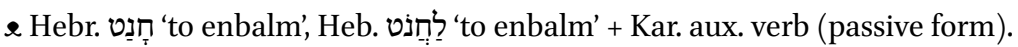

*haram 'stolen'

sen baqqyn bar ol ulustan tuvušlu elni qorquvčularny Tenrid́dan kerti elni hor etüvčülerni h. malny (Exo 18:21)

e Mod.SWKar. haram 'stolen'. Added by another hand in Exo 18:21.

harapla 'to insult'

-dy: ki sözün Tenrinin ol h.. (Deu 21:23)

\& Mod.SWKar. harapla- 'to insult'.

harbuz 'water-melon'

-larny: saġyndyq ošol ol balyqny ki ašaredik Micrida muft ošol ol hyjarlarny da ošol ol h. (Num 11:5)

e Mod.SWKar. harbuz 'water-melon'.

hargol 'a kind of locust; cricket (?)'

-nu: ošol bunlary alardan ašajyz ošol ol arbeni žynsysajyn da ošol sal'amny žynsysajyn da ošol ol h. ̧̌y̆ynsysajyn ošol ol hagavny žynsysajyn (Lev 11:22)

\& Heb. חחרִּוֹל 'a kind of locust', see Klein (1987: 230), Koehler and Baumgartner (1985: 331 .

hasebĺa- 'to number'

-ḿa: kim bolalyr h. dörtünčü ülüšün Jisra’elnin (Num 23:10)

e Mod.SWKar. haseple- 'to number'.

ḥasid 'pious'

Levi üčün ajtty tumimlerijni da urimlerijni symarladyj ḥ. kišije Aharonġa (Deu 33:8).

\& Heb. חָזִ 'pious'.

hasida 'stork'

-ny: ošol ol ḥ. ol anafany žynsysajyn da ošol duhifatny da ošol ol jary qanatyn (Lev 11:19); ol h. da ol anafany žynsysajyn da ol duhifatny da ol jary qanatyn (Deu 14:18)

e Heb. חִּסִידָה 'stork'. 
haskama qyl- 'to make an agreement, to agree' -dyjyz: bajladyjyz kiši ošol čerüv savutlaryn özünün da h. q. minḿa ol tavġa (Deu 1:41)

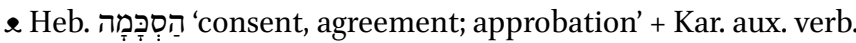

hašša: hašša bolġaj (dat., abl.) 'God forbid!, heaven protect!, be far from!'

h. b. saja qylmaqtan ošpu iš kibik öltürḿa cadiqni raša byla da bolgajdy nečik cadiq alaj raša' h. b. saja tör[e]čisimo bar ol jernin qylmastyr rast töra (Gen 18:25); da ajttylar anar nek sözlejdi bijim ošpu söz kibik h. b. qullaryja qylmaqtan ošpu iškibik (Gen 44:7); da ajtty h. b. maja qylmaqtan bunu ol kiši ki tabuldu ol čara qolunda (Gen 44:17)

e Mod.SWKar. hassa bolhaj ‘God forbid!', Mod.NWKar. hašča bolhej id., Mod.NWKar. hašče id.

ḥatat 'sin offering'

biširilmásin ḥamec ülüšlarin alarnyn berdim any otlu qarbanlarymdan qodeš qadašimdir ol ḥ. kibik da ašam kibik (Lev 6:10); sojulsun ol ḥ. alnynda Adonajnyn (Lev 6:18); bar h. ki kijirilśa qandan (Lev 6:23); nečik h. alaj ašam bir üvŕatüv alarġa (Lev 7:7); sojdu da h. qyldy (Lev 9:15); passim -lary: bar tirliklarisajyn da bar h. sajyn da bar ašamlarysajyn (Num 18:19) -laryn: juvuttular ošol h. (Lev 10:19); keltirdilar ošol qarbanlaryn özlarinin otlu qarban Adonajga da h. özlarinin alnyna Adonajnyn (Num 15:25)

-ny: sojsun ošol ol ḥ. ne orunda ki sojady ošol ol 'olany (Lev 4:29); sunsun ošol qolun bašy üstüna ol h. (Lev 4:33); andan sortun nečik endi qylmaqtan ol h. (Lev 9:22); neüčün ašamadyjyz ošol ol h. . (Lev 10:17); učardylar meni bular kibik da ašasajedim h. (Lev 10:19); passim

-nyn: qanyn tanasynyn ol h. töksün (Lev 4:7); ošol bar javyn tanasynyn ol h. ajyrsyn andan (Lev 4:8); qylsyn tanaġa ki nečik qyldy tanasyna ol h. (Lev 4:20); alsyn ol kohen qanyndan ol h. barmag்y byla (Lev 4:25); sunsun ošol qolun bašy üstüna ol ḥ. (Lev 4:29); passim

-qa: bir tana h. qylg்yn har kün ol bošatlyqlar üčün (Exo 29:36); balasyn syg்yrnyn tüǵalni Adonajġa h. (Lev 4:3); juvutsunlar ol qahal tana balasyn syġyrnyn h. (Lev 4:14); eger qozunu keltirśa qarbanyn özünün h. (Lev 4:32); sojsun any ḥ. ne orunda ki sojady ošol ol ólany (Lev 4:33); passim

-tan: ol bavurdan ol h. tütatti (Lev 9:10)

-ty: ošol jerinčiligin anyn küvdürgün otta tyšqartyn avulġa ḥ. ol (Exo 29:14); ne orunda ki sojady ošol ol 'olany alnynda Adonajnyn h. ol (Lev 4:24); ol qalgan qandan tamyzdyrylsyn bunjatyna ol mizbeahnnyn ḥ. ol (Lev 5:9); berḿasin anyn üstüna levona ki h. ol (Lev 5:11); jyštyrsyn aruv kiši ošol külün ol tanany bolsun šymatyna ulanlarynyn Jisra’elnin saqlavg்a bürkünür suvlarġa h. ol (Num 19:9)

-ydy: küvdürsün any ki nečik küvdürdü ošol burung் tanany h. ol qahalnyn ol (Lev 4:21) 
-yjny: qylg்yn ošol ḥ. da ošol 'olajny (Lev 9:7)

-yn: qylsyn ošol h. anyn da ošol olasyn (Num 6:16)

-yndan: ulaġyn ečkilarnin birni ḥatatqa bašqa ḥ. ol kipurimnin (Num 29:11)

-ynyn: qojmaqbyla bir keret jylda qanyndan h. (Exo 30:10);

e Heb. חַטָָ 'a sin offering’.

\section{hazair 'pig'}

-ni: ošol ol h. hota tujaqlajdy tujaq ol da ajyrady ajyryčyn tujaqnyn da ol güvün güvünlaḿajdi murdardyr ol sizǵa (Lev 11:7); ol h. hote tujaqlajdy esede tujaq ol da güvün keltirmejdi murdardy ol sizge (Deu 14:8)

e Heb. חִ זָ'pig', Mod.NWKar. hazzir 'pork'

*hem: *hem ki *'indeed'

da ajtty ol qatyng்a [h.] k. ajtty Tenri ašamajyz (Gen 3:1)

\& Cf. Heb. אַף 'Yea, has God said'.

herem 'thing devoted'

tek bar h. ki ḥerem etśa kiši Adonajga baryndan neki özünün (Lev 27:28); bar h. qodeš qadašimdir ol Adonajg̉a (Lev 27:28); bar h. ki herem etilśa ol adamdan julunmasyn (Lev 27:29); keltirmáain ḥor nerśani üvüja da bolursen h. kibik (Deu 7:6)

-den: jabušmasyn qoluja nemede ol h. (Deu 13:18)

-di: keltirmagin hor nerśani üvüja da bolursen herem kibik jiŕanči etḿa jiŕanči etkin any da ḥor etḿa ḥor etkin any ki ḥ. ol (Deu 7:6)

-nin: da bolur ol tüz čyqqanynda joveld́a qodeš Adonajġa tüzü kibik ol ḥ. kohenǵa bolsun tutuvlugu anyn (Lev 27:21)

^ Heb. חררֵם 'thing devoted'.

ḥeremli 'destroyed'

debeḥa etüvčü šejtanlarġa ḥ. bolsun ančaq Adonajg்a jalġyzg்a (Exo 22:19)

^ Heb. ח. ח1. destruction; 2. excommunication' + Kar. deriv. suff. $\Rightarrow$ ḥerem et-, ḥerem etil-

herem et- 'to devote'

-'armen: h. e. ošol šaharlaryn alarnyn (Num 21:2)

-śa: bar ḥerem ki h. e. kiši Adonajg̉a baryndan neki özünün (Lev 27:28)

-ti: tynlady Adonaj avazyna Jisra’elnin da berdi ošol ol Kena'anini da h. e. alarny da ošol šaharlaryn alarnyn (Num 21:3)

^ Heb. חִ 'thing devoted' + Kar. aux. verb. $\Rightarrow$ ḥeremli, ḥerem etil-

herem etil- 'to be devoted'

-ǵan: bar h. e. Jisra’eld́a saja bolsun (Num 18:14)

-śa: bar herem ki h. e. ol adamdan julunmasyn (Lev 27:29)

^ Heb. חִ 'thing devoted' + Kar. aux. verb (passive form). $\Rightarrow$ ḥeremli, ḥerem et-

ḥešboncet- *'to calculate oneself, to count oneself'

-sin: h. satynaluvčusu byla jylyndan satylmaġynyn anar jylynadejin ol jovelnin (Lev 25:50) 
\& Most likely a compound verb based on a Slavonic infinitive form in which the Pol. -ć or Russ. -mb infinitive ending or, more likely, the Pol. -ć się or Russ. -mbcr reflexive infinitive marker is replaced with Kar. $c$ and then the Karaim auxiliary verb et- is added to it. For similar forms cf., e.g., Mod.SWKar. zaspokojcet- 'to satisfy, to settle (payments)' < Pol. zaspokoić 'to satisfy' and Mod.SWKar. postaracet'to make effort' < Pol. postarać się id. (Németh 2011b: 327); see Németh 2011b: 91-94 for more examples and a detailed description of this phenomenon. We hypothesize the existence of a Pol. dial. (or jargon) ${ }^{* *}$ cheszbonić się 'to count yourself' based on

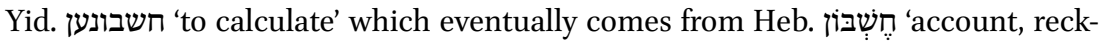
oning, calculation'. Its meanings 'to calculate' or 'to be calculated' are reconstructed on the basis of the context: it is used as the equivalent of Heb. לְחָשֵׁב 'to calculate', but a passive-reflexive verb 'to be counted' or 'to count oneself' fits in with the context as well. In other Western Karaim translations it is either NWKar. sanaš- 'lit. to count each other; to reckon with' (R) or WKar. haseblen- '1. to be counted; 2. to count oneself' (TKow.o1, H) that is used in this verse. In mss. $\mathrm{C}$ and $\mathrm{M}$, there is EKar. hesäp et- 'to count' used here. Hence, we reconstruct here a reflexive verb on the basis of the reciprocal and passive-reflexive forms used in the Western Karaim mss. R, TKow.o1, and H. Importantly, in the case of $\Rightarrow$ hešbontet- (with the medial $-t-)$, no passive-reflexive equivalents are used in other Karaim translations and a passive-reflexive interpretation of the verb is not allowed by the context, either. $\Rightarrow$ ḩešbontet-

ḥešbontet- *'to calculate, to count'

-sin: h. ošol jyllaryn satmag்ynyn da qajtarsyn ošol ol artadog்anny kišiǵa ki satty anar da qajtsyn tutuvlugiuna özünün (Lev 25:27); eger az qalsa jyllarda jylynadejin ol jovelnin da h. anar jyllaryna köŕa qajtarsyn ošol julunmaġyn özünün (Lev 25:52); eger ol joveld́an sortun qodeš etśa tüzün özünün da h. anar ol kohen ošol ol kümüšnü ol jyllarga köŕa ol qalġanlar jylynadejin ol jovelnin da eksilir qyjasyjdan (Lev 27:18); h. anar ol kohen ošol sahyn ol qyjasyjnyn jylynadejin ol jovelnin da bersin ošol ol qyjasyjnyn ol künd́a qodeš Adonajgia (Lev 27:23)

e Most likely a compound verb based on a Slavonic infinitive form in which Russ. $m b$ or Ukr. $-m u$ is replaced with Kar. $-t$ and then the Karaim auxiliary verb $e t$ - is added to it. For similar forms cf., e.g., Mod.SWKar. zaspokojtet- id. < Ukr. заспокоїти 'to satisfy; to soothe' (Németh 2011b: 327); see Németh 2011b: 91-94 for more examples and a detailed description of this phenomenon. We hypothesize the existence of a Ukr. dial. (or jargon) **хешбонити or a Russ. dial. (or jargon) *хешбонить based on Yid.

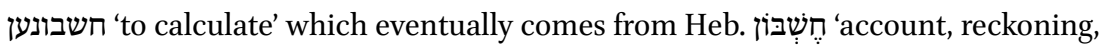
calculation'. Its meaning 'to calculate' is reconstructed on the basis of the context: it is used as the equivalent of Heb. לִחישֶׁב 'to calculate'; in other Karaim translations it is either NWKar. sana- 'to count' (R), WKar. haseble- id. (TKow.o1, H) or EKar. hesäp et-id. $(\mathrm{C}, \mathrm{M})$ which is used in the respective contexts. Any connection of this 
word to Mod.Heb. חֶשפבּוֹנִית 'receipt, invoice' is hardly probable for chronological reasons. $\Rightarrow$ ḥešboncet-

hin 'hin (a liquid measure)'

dazejtun javy h. (Exo 30:24); rast efa bolsun bürtük ölćaḿakḱa da rast h. bolsun sizǵa (Lev 19:36)

-nin: onunču ülüšü ol efanyn özek jumurulġan jančqan jav byla dörtünčü ülüšü ol $h$. da neseh dörtünčü ülüšü ol h. čag்yr ol bir qozuġa (Exo 29:40); čag்yr dörtünčü ülüšü ol h. (Lev 23:13); öźak onunču ülüšün ol efanyn jumurulgan dörtünčü ülüšü ol h.javbyla (Num 15:4); čag்yr nesehḱa dörtünčü ülüšü ol h. (Num 15:5); öźak eki 'esronlar jumurulgan javbyla üčünčü ülüšü ol h. (Num 15:6); passim

¿ Heb. הִ 'a liquid measure'.

holed 'mole'

budur sizǵa ol murdar qumuz da ol tebŕańadog்an ol jer üstüńa ol h. da ol 'ahbar da ol cav ̌̌ynsysajyn (Lev 11:29)

e Heb. חזלֶד 'mole'.

ḥomer 'homer (a name of a dry measure)'

eger tüzünd́an tutuvluġunun qodeš etśa kiši Adonajg̉a da bolsun qyjasyj urlugiuna köŕa tüz čačyladoġan urluq h. arpalar enli mitqal kümüška (Lev 27:16)

^ Heb. חמֶר 'homer (a name of a dry measure)'.

homet 'a kind of lizard; sand lizard (?)'

ol anaqa da ol kovah da ol leta’a da ol h. da ol tinšamet (Lev 11:30)

\& Heb. חמֶמט 'lizard; a kind of a lizard', see Klein (1987: 221), 'all reptiles that are not defined', Koehler \& Baumgartner (1985: 310).

ḥošen 'breastplate'

qylg̀yn ol h. üstüne synžyrlar čeklengen iši örkennin aruv altyndan (Exo 28:22); qylg̀yn ol h. üstüne eki jüzükler altyndan (Exo 28:23); kötürülmesin ol h. ol efod üstünden (Exo 28:28); kötürsün Aharon ošol atlaryn uvullarynyn Jisra’elnin h. ol mišpat byla jüregi üstüne (Exo 28:29); bergin ḥ. ol mišpatqa ošol ol urimni da ošol ol tumimni da bolsun jüregi üstüne Aharonnun (Exo 28:30); passim

-ge: šoham tašlary efodġa da milu’im tašlary h. (Exo 25:7); šoham tašlary efodg்a da milu’im tašlary h. . (Exo 35:9); ol nasiler keltirdiler ošol ol šoham tašlaryn efodg்a da ošol ol milu’im tašlaryn ḥ. (Exo 35:27) $\Rightarrow$-ǵa

-ǵa: qojdu anyn üstüna ošol ol hošenni da berdi ol ḥ. ošol ol urimni da ošol ol tumimni $($ Lev 8:8) $\Rightarrow$-ge

-ni: atlangyyztyrsynlar ošol ol h. jüzüklerinden anyn jüzükleri üstüne ol efodnun kök šnur byla (Exo 28:28); kijdirgin alarny Aharonġa ošol ol kölmekni da ošol qaftanyn ol efodnun da ošol ol efodnu da ošol ol ḥ. (Exo 29:5); qyldylar ošol ol h. iši ojuvlačunun (Exo 39:8); dört kül edi qatlanġan qyldylar ošol ol h. qaryš uzunlug்u da qaryš kenligi qatlanġan (Exo 39:9); atlanġyzdyrdylar ošol ol h. jüzüklerinden anyn jüzüklerińa ol efodnun kök šnur byla (Exo 39:21); passim 
-nin: bergin ošol eki ol jüzüklerni eki učlary üstüne ol ḥ. (Exo 28:33); bergin ošol eki altyn örkenlerni eki ol jüzükler üstüne učlaryna ol h. (Exo 28:24); qylg̀yn eki jüzükler altyndan da qojg̉un alarny eki učlary üstüne ol h. qyryj üstüne anyn ki janyna ol efodnun ičkerige (Exo 28:26); qyldylar eki altyn ojuvlar da eki jüzükler altyndan da berdiler ošol eki ol jüzüklerni eki učlary üstüne ol h. . (Exo 39:16); berdiler eki ol altyn erkenlerni eki ol jüzükler üstüne učlary üstüne ol h. (Exo 39:17); passim

e Heb. חשֶֶׁ 'breastplate of High Priest'. $\Rightarrow$ ḥošen mišpat

ḥošen mišpat 'brestplate of judgement'

qylg̀yn h. m. iši ojuvlavčunun iši kibik efodnun (Exo 28:15)

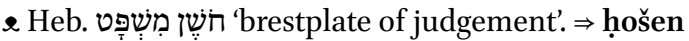

ḥökümlük: häoümlük byla 'oppressive'

qulluq ettirdilar Micrililer ošol ulanlaryn Jisra’elnin h. b. (Exo 1:13); erklanḿagin anda h. b. da qorqqun Tenrijdán (Lev 25:43); erklanḿagin anyn üstüna h. b. (Lev 25:46); jalčy kibik har jylda bolsun birǵasińa erklanmiasin anyn üstüńa ḥ. b. közlarijč́a (Lev 25:53)

e Mod.EKar. höküm '1. sentence; decree; 2. ruler', Mod.EKar. hökümder 'sovereign, ruler', see Aqtay \& Jankowski (2015). Used consistently as a translation of Heb. 'oppressively, ruthlessly'.

hüŭgat 'riddle'

-lar: aqyl aqylġa sözlarmen anyn byla da körümbyla da tüvül ḥ. byla (Num 12:8)

\& Mod.EKar. huǔ̌at huǔ̌žat 'riddle', see Aqtay \& Jankowski (2015: 188).

\section{I, ' I}

ij: ij qabullu|q 'pleasing aroma, aroma of acceptance'

tütetsin ol kohen ošol ol barysyn ol mizbeahta 'oladyr ol otlu qarban i. q. Adonajgia (Lev 1:9); otlu qarban i.q. Adonajġa (Lev 2:2); tütatsin olmizbeahta i.q. tütüsü anyn Adonajga (Lev 6:8); otlu qarban i. q. Adonajgia (Lev 23:18); qylma i. q. Adonajgja ol syg்rdan jemeśa ol qojdan (Num 15:3); passim

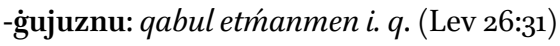

-nu: qabul etti Adonaj ošol ol i. q. (Gen 8:21);

-qa: tütetkin ol mizbeahta ol ola qatyna i. q. alnynda Adonajnyn otlu qarban ol Adonajga (Exo 29:25); qylgyn anar i. q. otlu qarban Adonajga (Exo 29:41); tütatsin alarny ol kohen ol mizbeahta ötmak otlu qarban i. q. bar jav Adonajga (Lev 3:16); 'oladyr ol i. q. (Lev 8:21); milu'imdirlar alar i. q. otlu qarban ol Adonajga (Lev 8:28); passim

e Mod.EKar. $i j$ 'aroma, fragrance'. $\Rightarrow$ qabulluq

ijilme $\mid \mathbf{k} *$ progeny'

-|gi: alg̀y̌̌ly bolur jemiši qursagjyjnyn da jemiši jerijnin da jemiši tuvaryjnyn i. ullu tuvarlaryjnyn da tišileri qojujnun (Deu 28:4); qarġyšly bolur jemiši qursaġyjnyn da jemiši jerijnin i. ullu tuvarlaryjnyn da tišileri qojujnun (Deu 28:18) 
-|gin: ašar jemišin tuvaryjnyn da jemišin jerijnin ta\{s\}polganyjadejin ki qaldyrmasty saja bürtük šarbet da saj i. ullu tuvarlaryjnyn da tišilerin qojujnun tasetkeninedejin seni (Deu 28:51)

-|ginin: ašyrg்yn bar ačylmag்yn qursaqnyn Adonajg̉a da bar ačylmag்yn i. tuvarnyn ki bolsa saja ol erkekler Adonajg̈a (Exo 13:12)

e Mod.NWKar. ijil- 'to be sent; to be freed', cf. Mod.SWKar. siriv 'progeny' in H (Deu $7: 13) . \Rightarrow$ ijilmák

ijilma|k *'progeny'

-|gin: süv́ar seni da alġy̌̌lar seni da arttyryr seni da alg்yšlar jemišin qursag்yjnyn da jemišin jerïnin bürtügüjnü da šarbetijni da sajyjny i. ullu tuvarlaryjnyn da tišilarin qojujnun ol jer üstüńa ki antetti atalaryja berḿa saja (Deu 7:13)

^ Mod.NWKar. ijil- 'to be sent; to be freed', cf. Mod.SWKar. siriv 'progeny' in H (Deu $7: 13) . \Rightarrow$ ijilmek

ijir- 'to spin'

-diler: bar qatyn uslu jürekli qollarybyla i. (Exo 35:25); bar ol qatynlar ki qunušturdu jürekleri alarnyn alarny usbyla i. ošol junun ol ečkilernin (Exo 35:26)

e Mod.SWKar. ijir- 'to spin'. $\Rightarrow$ *ijirgen

*ijirgen 'yarn; that which is spun'

-ni: bar qatyn uslu jürekli qollarybyla ijirdiler da keltirdiler ijir [genni] ošol ol köknü da ošol jipkinni da ošol qyrmyzy jipekni da ošol ol bezni (Exo 35:25)

e Mod.SWKar. ijirgen 'yarn'. $\Rightarrow$ ijir-

ilk 'finest, best'

sen alg̀yn özüje i. otjamlar (Exo 30:23)

e Mod.NWKar. ilk 'first', Mod.SWKar. ilk 'first; superior'.

ingir: eki ingirler arasyna eki ingirlar arasyna 'at twilight'

sojsunlar any bar qahaly šymaty Jisra'elnin ol e. ingirler a. (Exo 12:6); ol e. ingirler a. ašarsyz et da ertenbylada tojarsiz ötmekten da bilirsiz ki menmen Adonaj Tenrijiz (Exo 16:12); ošol ol ekinči qozunu qylġyn ol e. ingirler a. tirkisikibik ol ertenbylanyn (Exo 29:41); ol e. ingirlar a. tütetsin any (Exo 30:8); ol burung் janġajda ondörtünčü kününd́a janġajnyn ol e. ingirlar a. qarbany Pesahnnyn Adonajġa (Lev 23:5); tüzüsün any alnynda Adonajnyn hammeśa ol e. ingirlar a. tütatkanind́an sortun ol tütünü (Lev 24:8); ondörtünčü kününd́a ošpu janġajnyn ol e. ingirlar a. qylyjyz any (Num 9:3); passim

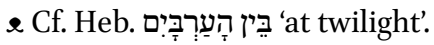

'isaron 'one tenth, tenth part'

da bir 'i. öźak jumurulgan javbyla tirkiǵa da bir log் jav (Lev 14:21); birar 'i. har bir qočqarg்a ondört qozularġa (Num 29:15); bir 'i. öźak tirki jumurulgan javbyla har bir qozuġa 'ola ij qabulluq otlu qarban Adonajga (Num 28:13); birar i. qylgynn har bir qozuġa jedisid́a ol qozularg்a (Num 28:22); birar i. har bir qozuġa jedisid́a ol qozularga (Num 28:29); passim 
-dan: alġyn öźak da biširgin any on eki halalar ekśar i. bolsun ol bir hala (Lev 24:5)

\& Heb. עִשָׁiֹ 'one tenth, tenth part'. $\Rightarrow$ 'esron

issin- 'to conceive (said of animals), to copulate'

-dilar: da i. ol qoj baġadog்ač ol tajaqlar üstüńa da töredilar ol qoj čybarlarny bürtüklülerni da alalylarny (Gen 3o:39)

-ǵan: da edi bar i. vaḥtta ol qoj ol jazdagjylar (Gen 30:41); edi i. vahtta ol qoj (Gen 31:10) -iredilar: ki kelsejdilar ol qoj ičma uturusuna ol qojnun i. kelgenlerind́a ičma (Gen 30:38)

e Mod.NWKar. issiń- 'to get hot, to warm oneself', cf. Heb. ירָי 'to be hot; to conceive, to breed'.

jabunur 'quilt'

-ujnun: jalavlar qylġyn özüje dört müvüšler üstüne j. kijabunsaj anyn byla (Deu 22:12) -un: juvar čaġyrbyla upraġyn da qanynbyla borlalarnynj. (Gen 49:11)

e Mod.SWKar. jabunur 'quilt'.

jahaslan- 'to be confirmed, to be proved'

-dylar: ošol bar ol క̌ymatny jyštyrdylar burung்u kününd́a ol ekinči janġajnyn da j. uruvlarysajyn (Num 1:18)

e Mod.SWKar. jahaslan- 'to confirm (with documents), to prove'.

jančqyč 'mortar'

-ta: jajylyredilar ol ulus da jyštyryredilar da tartaredilar tijirḿand́a jemeśa jančaredilar j. da biširiredilar čölmátía jemeśa qylaredilar jajmalar da boluredi tatuvu anyn tatuvu kibik semizliginin ol javnyn (Num 11:8)

e Mod.NWKar. jančhyč 'pestle'.

janšuf 'long-eared owl'

-nu: ošol ol kosnu da ošol ol šalahny da ošol olj. (Lev 11:17); ošol ol kosnu da ošol ol j. da ol tinšemetni (Deu 14:16)

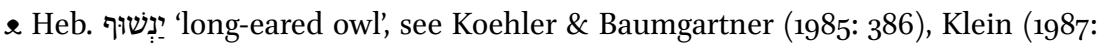
260).

jajačy 'bowman, archer'

da edi bolušlug̉u Tenrinin ol ulanbyla da ulgajdy da olturdu midbarda da edi oq atuvču daj. (Gen 21:20)

e Mod.NWKar. jaja 'bow' + deriv. suff.

*jal- *'to tie'

-a: kijirilebiz syltav etḿa üstümüzǵa da j.japma üstümüzǵa da alma bizni qullarġa da ošol ěseklerimizni (Gen 43:18)

e Mod.SWKar. jalav 'string, rope', perhaps a derivative of Kar. *jala-, cf. *jalya- '1. to tie; 2. to lie' of Mongolic origin according to Räsänen (1969: 183). $\Rightarrow$ jalav 
jalan 'smooth (not hairy)'

ajtty Ja'aqov Riveqag̉a anasyna muna 'Esav qaryndašym tüklü kišidi da men j. kišimen (Gen 27:11)

e Mod.NWKar. jalan 'naked'.

jalav 'string'

-lar: j. qylg̀nn özüje dört müvüšler üstüne jabunurujnun (Deu 22:12)

e Mod.SWKar. jalav 'string, rope'. $\Rightarrow$ *jal-

jaman 'wretchedness'

-ymny: eger bulaj qyladeśaj sen maja öltürgün meni \{endi\} öltürḿa eger taptym eśa širinlik 'enajetlaryjda da körmáajim j. (Num 11:15)

e Mod.NWKar. jaman 'evil, bad', cf. BHeb. רָזָה '1. evil, 2. wickedness; 3. misery, trouble'. $\Rightarrow$ jamanly

jamanlyq 'wretchedness'

alsajyz dag்yn ošol bunu qatymdan da učrasa any ölüm da endirirsiz ošol pirligimni j. byla görǵa (Gen 44:29)

e Mod.NWKar. jamanlyḥ 'evil', cf. BHeb. רָָּה 1. evil, 2. wickedness; 3. misery, trouble'. $\Rightarrow$ jaman

janbaš 'bedhead'

-lar: urar seni Adonaj jaman čyban byla ol tizler üstüne da ol j. üstüne (Deu 28:

35)

e Mod.EKar. janbaš 'bedhead'.

jangaj 'month'

ajtty qaryndašy anyn da anasy anyn oltursun ol qyz birgemizǵa jyl jemeśa on j. andan sortun baryr (Gen 24:55); ajtty anar Lavan tek süvegim da gufumdur sen da olturdu birgesińa j. künlar (Gen 29:14); j. ošpu sizge bašydy janġajlarnyn burunġudu ol sizge janġajlaryna ol jylnyn (Exo 12:2); jemeśa eki kün jemeśa j. jemeśa jyl uzaq turġanda ol bulut ol miškan üstüna tohtama anyn üstüna (Num 9:22); j. künlarǵadejin (Num 11:20); passim

-da: alty jüzünčü jylda tirliklarina Novaḥnyn ol ekinči j. ol jedinči kününd́a janġajnyn ošpu künd́a (Gen 7:11); da tohtady ol gerep [ol jed] inči j. on jedinči kününd́a janġajnyn tavlary [üst] üńa Araratnyn (Gen 8:4); edi alty jüz birinči jylda burunġuj. burunġu kününd́a janġajnyn (Gen 8:13); ol ekinči j. egirmi jedinči [kün] ünd́a janġajnyn qurudu ol jer (Gen 8:14); burung்u j. ond[ö]rtünčü kününde janġajnyn (Exo 12:18); passim

-ġa: ol suvlar baryredilar [da e]ksiliredilar ol onunčuj. dejin onunču jangajda [burun] gu kününde janġajnyn köründülar bašlary ol tavlarnyn (Gen 8:5)

-lar: hamila boldu ol \{[q]atyn $\}$ da töredi uvul da kördü any ki jaḩ̌ýdy ol da astrady any üčj. (Exo 2:2)

-larnyn: janġaj ošpu sizge bašydyj. burunġudu ol sizge janġajlaryna ol jylnyn (Exo $12: 2$ ) 
-laryna: janġaj ošpu sizge bašydy janġajlarnyn burung்udu ol sizge j. ol jylnyn (Exo 12:2); budur 'olasy janġajnyn jangajynda j. ol jylnyn (Num 28:14)

-nyn: alty jüzünčü jylda tirliklarina Novaḥnyn ol ekinči janġajda ol jedinči kününd́aj. ošpu künd́a (Gen 7:11); da tohtady ol gerep [ol jed] inči janġajda on jedinči kününd́a j. tavlary [üst] üńa Araratnyn (Gen 8:4); ol suvlar baryredilar [da e]ksiliredilar ol onunču janġajgadejin onunču janġajda [burun] $\dot{g}$ u kününde j. köründülar bašlary ol tavlarnyn (Gen 8:5); edi alty jüz birinči jylda burunġu janġajda burung் kününd́aj. (Gen 8:13); ol ekinči janġajda egirmi jedinči [kün] ünd́a j. qurudu ol jer (Gen 8:14); passim

e Mod.NWKar. janhaj 'new moon'.

jaram *'wound'

eger kleśam öltürḿa kišini ačyq j. byla öltürürmen any (Gen 4:23); da ulanny öltürürmen bütüvde j. byla (Gen 4:23)

e Mod.NWKar. jar- 'to break, to crash, to crack', Mod.NWKar. jara- 'wound'.

jaramas 'disgraceful'

čačmag்yn borlalyg்yjny eki žyns mag்at j. bolur saja ol jemiš (Deu 22:9); bolalmasyn eri anyn ol burungu ki ijdi any qajtma alma any özüne qatynlyqqa andan sortun ki j. bolundu (Deu 24:4)

\& Mod.EKar. jaramaz 'bad; unworthy, disgraceful; dissolute'.

jaratylmyš: sondraġy jaratylmyšlar 'back (of God)'

-ymny: ketirirmen ošol bujrug்umnu da körersen ošol s.j. da qyblalarym körünmesler (Exo 33:23)

e Mod.NWKar. sondrahy 'last', Mod.NWKar. juvuzdan jaratylmyš 'back (of God)', see

A. Zajączkowski (1929: 13).

jarty: jarty ol kečede $\sim$ jarty ol kečed́a 'at midnight'

bulaj ajtty Adonaj j. o. kečed́a tekli menim bujrug்um čyg்ar ortasynda Micrinin (Exo 11:4); da edij. o. kečede da Adonaj qyrdy bar tunġučnu jerind́a Micrinin (Exo 12:29)

e Mod.NWKar. jarty keč́a 'midnight'.

jary qanaty 'bat'

-n: ošol ol ḥasidany ol anafany క̌ynsysajyn da ošol duhifatny da ošol ol j. q. (Lev 11:19); ol hasidany da ol anafany žynsysajyn da ol duhifatny da ol j. q. (Deu 14:18)

\& Mod.SWKar. jarykanatly 'bat', Mod.EKar. jarqanat id.

jas 'mourning'

ajtty 'Esav kölnünd́a juvusalar j. künleri atamnyn öltürürmen Ja'aqovnu qaryndašymny (Gen 27:41); syjyt ettilar anda ullu syjyt da küčlü astry da qyldy atasy üčün j. jedi künler (Gen 50:10)

-ny: kördü olturuvčusu ol jernin ol Kenáannyn ošol ol j. (Gen 50:11)

-tyr: ajttylar küčlüj. bu Micriǵa (Gen 50:11)

-ynyn: da tüǵandilarjylamaq künlarij. Mošenin (Deu 34:8)

e Mod.NWKar. jasly 'adj. mourning; mourner'. 
jasangan '1. armed; 2. armed man, soldier'

ašsa siznin bar j. ol Jarden ašyra (Num 32:21); eger ašsalar ulanlary Gadnyn da ulanlary Ru’uvennin birǵajizǵa ol Jarden ašyra bar j. čerüvǵa alnynda Adonajnyn (Num 32:29)

-lar: da j. čyqtylar ulanlary Jisra’elnin jerind́an Micrinin (Exo 13:18); eger ašamasalar j. birǵajizǵa (Num 32:30); ašarbizj. alnynda Adonajnyn jerińa Kena’annyn (Num 32:32); Tenrijiz berdi sizǵa ošol ol jerni ošpunu meŕaslaḿa any j. ašyjyz alnynda qaryndašlaryjyznyn ulanlarynyn Jisra’elnin bar tuvušlu el (Deu 3:18); bargjajlar j. čerüvge alnynda qaryndašlarynyn (Deu 33:21)

-lary: symarlandylar minlarind́an Jisra’elnin mińar kiši ševett́an on eki min j. čerüvnün (Num 31:5)

-y: qullaryj ašarlar bar j. čerüunün alnynda Adonajnyn čerüvǵa ki nečik bijim sözlajdi (Num 32:27)

e Mod.SWKar. jasanhan '1. armed; 2. soldier'.

jasly: jasly bol- 'to mourn'

-du: jyrtty Ja'aqov upraqlaryn da qojdu kijiz bellerind́a da j. b. uvlu üčün köp künlar (Gen 37:34)

-dular: ešitti ol ulus ošol ol jaman söznü ošpunu da j. b. (Exo 33:4); sözladi Moše ošol ol sözlarni ošpularny bar ulanlaryna Jisra'elnin da j. b. ol ulus astry (Num 14:39)

e Mod.NWKar. jasly 'adj. mourning; mourner’ + aux. verb.

jasty $\mid \mathbf{q}$ 'saddle'

-|g்yna: Rahelel aldy ošol ol sturlaplarny da qojdu alarnyj. ol tevenin (Gen 31:34)

^ Mod.NWKar. jastyh 'pillow', Mod.SWKar. jastyk 'saddle'.

jašfe 'jasper'

ol dörtünčü jerge taršiš tašy da šoham daj. (Exo 28:20); ol dörtünčü jerge taršiš šoham daj. (Exo 39:13)

\& Heb. יִיְשפָה 'jasper'.

jašyl 'green'

bolsa ol hastalyqj. syman (Lev 13:49); jerǵaĺar j. symanlar (Lev 14:37)

e Mod.NWKar. ješil 'green'.

jatuv 'intercourse'

-un: qatyn ki jatsa kiši anyn byla j. urluqnun da juvsunlar guflaryn suvbyla da murdar bolsunlar ol ingirǵadejin (Lev 15:18); kiši ki jatsa qatyn byla j. urluqnun (Lev 19:20); jatsa kiši anyn bylaj. urluqnun dajašyryn bolsa bu nerśa közlarind́an erinin (Num 5:13) -una: öltürüjüz bar erkakni jašta da bar qatynny bilüvčünü kišini j. erkaknin öltürüjüz (Num 31:17)

e Mod.NWKar. jatuv ‘. bed; 2. lying', urluq 'seed, semen; offspring'. jav 'oil'

qojdu any maceva da qujdu j. bašyüstüńa anyn (Gen 28:18); q[u]jdu anyn üstüńa neseh da $q[u]$ jdu anyn üstüńa j. (Gen 35:14); j.jaryqlyqqa otjamlar oljaġynmaqjavġa 
da tütüsüne ol otjamlarnyn (Exo 25:6); da halalar macalar jumurulganlar j. byla da juġa macalar jag்l janlar j. byla (Exo 29:2); jumurulgan jančqan j. byla (Exo 29:40); passim

-dan: alg̀yn ol qandan ol mizbeah üstüne da jag்ynmaq j. da bürkkün Aharon üstüne (Exo 29:21); qojdu ol jaġynmaq j. bašy üstüna Aharonnun (Lev 8:12); aldy Moše ol jag்ynmaqj. da ol qandan ki ol mizbeạ üstüna da bürktü Aharon üstüna (Lev 8:30); alsyn ol kohen log் ol j. (Lev 14:15); mančsyn ol kohen ošol barmag்y ol on qolunun ol j. (Lev 14:16); passim

-ga: jav jaryqlyqqa otjamlar ol jag்ynmaq j. da tütüsüne ol otjamlarnyn (Exo 25:6); otjamlar ol jaġynmaqj. da tütüsüne ol otjamlarnyn (Exo 35:8); ošol ol otjamny da ošol ol javny jaryqlyqqa da ol jaġynmaqj. da tütüsüne ol otjamlarnyn (Exo 35:28); mančar j. ajaġyn (Deu 33:24)

-ny: alg்yn ošol ol jag்ynmaqj. (Exo 29:7); ošol ol jag்ynmaqj. da ošol tütüsün ol otjamlarnyn qodeške barča nečik ki bujurdum saja alaj qylsynlar (Exo 31:11); ol jag̉ynmaqj. da ošol tütüsün ol otjamlarnyn (Exo 35:15); ošol ol otjamny da ošol ol j. jaryqlyqqa da ol jag்ynmaq javġa da tütüsüne ol otjamlarnyn (Exo 35:28); qyldy ošol ol jag்ynmaq j. aziz (Exo 37:29); passim

-y: alsynlar saja zejtunj. aruvnu jančqanny jaryqlyqqajandyrma čyraq hammeše (Exo 27:20); zejtun j. hin (Exo 30:24); jaġynmaqj. Adonajnyn üstüjüzd́a (Lev 10:7); qujulsa bašy üstüńa anyn ol jaġynmaqj. (Lev 21:10); alsynlar saja zejtun j. aruvnu (Lev 24:2); passim

-yn: da ošol j. ol jaryqlyqnyn (Exo 35:14); da ošol j. ol jaryqlyqnyn (Exo 39:37); bar j. sajnyn da barj. šarbetnin (Num 18:12)

-yndan: ajyrsyn andan tolu uvuču öźagind́an daj. anyn bar levonasy byla (Lev 2:2); da j. anyn (Lev 2:16); ajyrsyn andan qoš uvuču byla öźagind́an ol ti [rki]nin da j. anyn da ošol bar ol levonany (Lev 6:8)

-ynyn: tačy jaġynmaqj. Tenrisinin anyn üstüńa (Lev 21:12)

e Mod.NWKar. jav 'fat; butter; tallow'.

jecer 'character; inclination'

kij.jüreginin ol adamnyn [jamandy] (Gen 8:21)

-in: bilemen ošolj. anyn (Deu 31:21)

\& Heb. יצֶֶ.. '1. purpose; 2. inclination', Mod.SWKar. jecer 'character'.

jecer hara 'evil inclination'

-nyn: ošol jüregin urlugiujnun ki ol remezdi eksilmegine j. h. (Deu 3o:6)

\& Heb. יִצֶר הַרֵע 'evil inclination'.

jemiš ag̉ačy $\Rightarrow$ ag̉ač

jengillet- 'to make easier'

-kin: bar ol kiči išni töre etsinler özleri da j. üstüjdán da kötürsünler birgeja (Exo 18:22)

e Mod.SWKar. jengillet- 'to ease, to make easier'. 
jerǵa 'deck (in a ship)'

-ler: j. eki qat da üč qat qylg̀yn any (Gen 6:16)

e Mod.WWKar. jerǵa 'degree; step; row, layer'.

jerenčili|k 'dung'

-gin: ošol etin ol tananyn da ošol terisin anyn da ošol j. anyn küvdürgün otta tyšqartyn avulga hatatty ol (Exo 29:14)

e Mod.NWKar. jereńčilik (!) 'dirt; manure’, Mod.NWKar. jerańćilik 'abomination'. jesi: oq jesi|si 'archer'

-|leri: ačy ettilar anar da attylar any dušman boldular anar o.j. (Gen 49:23)

\& Mod.NWKar. ok 'arrow', Mod.NWKar. jesi 'man, owner'.

ješillik 'a kind of fungal disease; probably mildew, mould (lit. greenness)'

urar seni Adonaj suvuq hastalyq byla da issi hastalyq byla da küvmek byla da qararmaq byla da qurumaq byla da sarylyqbyla daj. byla da quvarlar senitaspolganyjadejin (Deu 28:22)

\& Mod.NWKar. ješillik' '1. the green colour; 2. grass' (cf. the green colour of mould), cf. BHeb. ירָקוֹן '1. paleness; 2. mildew', see Klein (1987: 265) and Koehler \& Baumgartner (1985: 406), BHeb. ירָז 'pale; green’ and Heb. ירק '1. (PBHeb.) to become yellow, pale; 2. (Mod.Heb.) to become green', see Klein (1987: 265) and Koehler \& Baumgartner (1985: 406).

jetilmek 'ripening'

-lerin: hyy̌̌yn Šavu'otnun qylg̀yn özüje j. ormaqlarynyn budajlarnyn (Exo 34:22)

-lerinin: hyž̌n ol ormaqnynj. išlerijnin (Exo 23:16)

\& Mod.NWKar. jetil- 'to ripen', Mod.SWKar. jetilmek 'ripeness'.

jetkili|k: jetkiligine köre 'to the best of one's abilities, as best as one can'

kördüler ošol šehinasyn ol Tenrinin j. k. aqyllarynyn (Exo 24:11); berne bergin anarj. k. küčünün qolujnun (Deu 15:17)

\& Mod.EKar. jetkiliginče 'as best as one can, sufficiently', Mod.SWKar. jetkiligine kere 'to the best of one's abilities', see Németh (2011b: 290).

jiŕanči 'abominable'

ajipin qatynnyn da qyzynyn aškartmag்yn ošol qyzyn uvlunun da ošol qyzyn qyzynyn almaġyn aškartmaġyn ajipin anyn juvuqtular alar j. išti ol (Lev 18:17); erkakbyla jatmaġyn jatuvlaryn qatynnyn j. išti ol (Lev 18:22); heč tuvarda bermagin jatuvujnu murdar bolma anyn byla da qatyn turmasyn alnynda tuvarnyn qošulmaj. išti ol (Lev 18:23); saqlajyz siz ošol resimlarimni da ošol törálarimni da qylmajyz bar olj. išlard́an ošpulardan ol jerli da ol garip ol tirilüvčü ortajyzda (Lev 18:26); ošol bar ol j. išlarni ošpularny qyldylar eli ol jernin ki edilar sizd́an burun da murdar boldu ol jer (Lev 18:27); passim

e Mod.NWKar. jerińči jereńči jerańči 'disgusting, abominable’.

jiŕanči et- 'to find repulsive'

-ijiz: da ošol gövd́aĺarin alarnynj. e. (Lev 11:11); ošol bunu j. e. ol quštan (Lev 11:13) 
-kin: keltirḿagin hor nerśani üvüja da bolursen herem kibik jiŕanči etḿa j. e. any da hor etría hor etkin any ki heremdi ol (Deu 7:26)

-ma: keltirmagin hor nerśani üvüja da bolursen herem kibik j. e. jiŕanči etkin any da hor etḿa hor etkin any ki heremdi ol (Deu 7:26)

-majiz: j. e. ošol žanlaryjyzny bar ol qumuzbyla (Lev 11:43); da j. e. ošol žanlaryjyzny tuvarbyla (Lev 20:25)

e Mod.NWKar. jeráńč et- 'to find repulsive'.

jiŕančili|k 'dung'

-|gi: küvdürsün ol tanany közlarič́a anyn ošol terisin anyn da ošol etin anyn da ošol qanyn anyn j. byla küvdürsün (Num 19:5)

-|gin: ošol terisin ol tananyn da ošol bar etin anyn bašybyla da tizlaribyla da ičin anyn daj. anyn (Lev 4:11); ošol ol tanany da ošol terisin anyn da ošol etin anyn da ošolj. anyn küvdürdü otta (Lev 8:17)

-ĺarin: ošol terilarin alarnyn da ošol etlarin da ošolj. alarnyn (Lev 16:27)

e Mod.NWKar. jereńčilik (!) 'dirt; manure’, Mod.NWKar. iŕańčilik jeŕańčilik 'abomination'.

\section{Jisra'elka 'Hebrew women'}

čyqty uvlu J. qatynnyn da ol edi uvlu Micrili kišinin (Lev 24:10); belgiladi uvlu ol J. qatynnyn ošol atyn ol Tenrinin (Lev 24:11); satylsa saja qaryndašyj ol Jisra'el jemese ol J. da qulluq etsin saja altyjyllar (Deu 15:12)

-lar: tüvüldü ol Micrili qatynlar kibik ol J. ki savdylar alar kelmesten burun alarġa ol anača da törejdilar (Exo 1:19)

-lardan: ündejim saja ömüzdürüvčü qatyn olJ. da ömüzdürsün saja ošol ol ulanny (Exo 2:7)

-larny: ajtty par'o töretkenijizde ošololJ. da bag்yjyz ol törer orun üstüne eger uvul bolsa ol da öltürüjüz any (Exo 1:16)

-nyn: da uruštular avulda uvlu ol J. da ol Jisra’el kiši (Lev 24:10)

e Mod.NWKar. Jisrael 'Israelites' + deriv. suff.

jog̀a 'flan'

boḥon ötmek bir da hala ötmek javly bir daj. bir četeninden ol macalarnyn ki alnynda Adonajnyn (Exo 29:23)

\& Mod.NWKar. juga 'flan'; the presence of -o- in joga, instead of jug $a$, might also be a scribal error.

jomulma $\mid \mathbf{q}$ 'concentration'

-|'̇y: sung்n qolujnu suvlary üstüna Micrinin özenleri üstüne Nilleri üstüne da ozeralary üstüne da bar j. üstüne suvlarynyn da bolsunlar qan (Exo 7:19)

e Mod.SWKar. jomulmak 'cluster, concentration'.

jovel 'jubilee'

j.jylydy ol aziz bolsun sizǵa ol tüzd́an ašajyz ošol bitišin anyn (Lev 25:12); ki nečik bolsa olj. ulanlaryna Jisra'elnin (Num 36:4) 
-di: j. oljyly ol enlinči jylnyn (Lev 25:11)

-dir: aziz tutujuz ošol jylyn ol enlinči jylnyn da čag்yryjyz azatlyq jerd́a bar olturuvčularyna anyn j. ol (Lev 25:10)

-da: jylynadejin ol jovelnin da čyqsyn j. da qajtsyn tutuvluginna özünün (Lev 25:28); eger julunmasa tolg̀unča anar tüǵal jyl da qajjam bolur ol üv ki šaharda ki anar qala ovellikḱa satyn aluvčuġa any dorlarysajyn čyqmasyn j. d́a (Lev 25:30); üvlari ol azbarlarnyn ki johtur alarġa qala čüvŕa tüzü ornuna ol jernin sagyyšlansyn julunmaq bolsun anar da j. čyqsyn (Lev 25:31); ki julusa ol Levilard́an da čyqsa satmag்y üvnün da šahary tutuvlugiunun j. (Lev 25:33); bolur ol tüz čyqqanynda j. qodeš Adonajg̈a (Lev 27:21)

-d́an: sanybyla jyllarnyn ol j. sortun (Lev 25:15); eger ol j. sortun qodeš etśa tüzün özünün (Lev 27:18)

-ge: alġyn ošol ol bizni da bergin qulaġyna anyn da ešikke da bolur saja qul j. dejin (Deu 15:17)

-nin: qulluq etsin anar dunjaġadejin ki oldujylyj. (Exo 21:6); jylynda ol j. ošpu qajtyjyz har kiši öz tutuvluġuna (Lev 25:13); jylynadejin ol j. da čyqsyn joveld́a da qajtsyn tutuvluġuna özünün (Lev 25:28); očarkibik bolsun birǵaja jylynadejin ol j. qulluq etsin birǵaja (Lev 25:40); ḥešbon četsin satynaluvčusu byla jylyndan satylmag்ynyn anar jylynadejin olj. (Lev 25:50); passim

e Mod.SWKar. jovel 'jubilee', Heb. יוֹיבֶ 'jubilee, year of jubilee'. $\Rightarrow$ jovel: jovelge dejin, ovellikḱa

jovel: jovelge dejin 'forever'

alġyn ošol ol bizni da bergin qulaġyna anyn da ešikke da bolur saja qul j. d. (Deu 15:17)

\& Mod.SWKar. jovel 'jubilee', Heb. יוֹבר 'jubilee, year of jubilee' $\Rightarrow$ jovel, ovellikḱa

jovšem 'all the more'

da j. Lemeh üčün ki (Gen 3:24); bügün tanuvčular edijiz alnynda Adonajnyn da j. ki ölgenimden sortun (Deu 31:27)

\& OPol. $i$ owszem '1. furthermore, what is more, and even; 2. especially, particularly'.

jugia 'flat'

ošol mačmarlaryn ol jazyqlylarnyn ošpularnyn žanlarybyla da qylsynlar alardan j. blahalar qaplav mizbeahqqa (Num 17:3)

\& Mod.NWKar. juhart- 'to flatten'.

julquv 'baldness, bald place'

julqmasynlar j. bašlarynda da qyryjyn saġallarynyn julumasynlar da guflarynda čyzmasynlar čyzmaq (Lev 21:5); ulanlardyrsiz Adonajġa Tenrijizge čyzylmajyz da qojmajyzj. közlerïiz arasyna ölü üčünde (Deu 14:1)

e Mod.NWKar. julh- 'to pluck, to pull out', Mod.NWKar. julhu- 'to pluck' + deriv. suff. 
juluvun al- 'to redeem'

-g̀yn: tek juluvun alma j. a. tung்učunun ol adamnyn da ošol tunġučunun ol murdar tuvarnyn juluvun alg̀yn (Num 18:15); juluvlary anyn aj jaštan j. a. qyjasyj byla kümǚ̌ beš mitqallar mitqaly byla ol qodešnin igirmi gögdü ol (Num 18:16)

-ma: tek j. a. juluvun alġyn tung்učunun ol adamnyn da ošol tunġučunun ol murdar tuvarnyn juluvun algyn (Num 18:15)

-maġyn: tung்učunun ögüznün jemeśa tung்učunun qozunun jemeśa tung்učunun ečkinin j. a. azizdi alar (Num 18:17)

e Mod.NWKar. juluv 'redemption', Mod.NWKar. al- 'to take'.

jumušlan- 'to get profit'

-maġyn: eger süvmesej any da ïgin any öz erkińa da satma satmag்yn any kümüške j. satuvbyla anyn byla anyn üčün ki qyjnadyj any (Deu 21:14)

-sa: ki tabulsa kiši urlavču žanny qaryndašlaryndan ulanlaryndan Jisra’elnin da savut išibyla j. anda da satsa any (Deu 24:7)

\& Mod.SWKar. jumuslan- 'to use, to benefit'.

jük: jük kötür- 'to bear burden, to be weighed down by a burden'

-me: qajyrdy javrunun j. k.jügün tora oḥumaqnyn (Gen 49:15) $\Rightarrow$-ma

-ma: budur qullug்u uruvlarynyn ol Geršoninin qulluq etŕa da j. k. (Num 4:24) $\Rightarrow$-me

e Mod.NWKar. juk 'burden, load', Mod.NWKar. kotur- 'to carry'.

jülü- 'to shave'

-masin: jülünsün da ošol ol neteqni j. (Lev 13:33)

e Mod.NWKar. ülu- 'to shave'. $\Rightarrow$ jülün-, jülüt-

jülün- 'to shave (onself)'

-sün: j. da ošol ol neteqni jülümáasin (Lev 13:33)

e Mod.NWKar. üluń- 'to shave (onself)'. $\Rightarrow$ jülü-, jülüt-

jülüt- 'to let shave'

-tü: j. da tüvšürdü ošol upraqlaryn da keldi par'oġa (Gen 41:13)

e Mod.NWKar. ülu- 'to shave.' $\Rightarrow$ jülü-, jülün-

jürüšlü 'living, alive'

j. süvekti süveklerimd́an (Gen 2:23)

^ Mod.EKar. jürüš 'life' + deriv. suff.

jüvüš: jüvüš ölčüv 'liquid measure'

-d́a: qylmajyz avanlyq kečinmiaklik nerśalard́a bürtkün ölčüvünd́a kümüš ölčüvünd́a daj. ö. (Lev 19:35)

e Mod.EKar. jüvüš 'wet'.

jyg்ys 'impure'

kiši ki jatsa hastaj. qatyn byla (Lev 20:18)

\& Mod.NWKar. jyhyz 'impurity'.

jyġysly|q 'menstruation'

eger qyz ulanny töŕaśa da murdar bolsun eki jedilar j. murdarlyg்y kibik (Lev 12:5); 
barča neki jatsa anyn üstüna ol qatyn j. vahtynda murdar bolsun (Lev 15:20); qatyng்a j. vahtynda murdarlyg்ynyn juvumag்yn aškartma ajipin anyn (Lev 18:19)

-|ġynda: qatyn ki bolsa zavly qan bolsa aqmag்y anyn gufundan jedi künlar bolsun j. (Lev 15:19); hasta qatynnyn j. da ol zavly\{nyn\} zavlyg்ynda erkaknin da qatyn kišinin da kišinin kijatsa murdar qatyn byla (Lev 15:33)

-|ġynyn: murdar bolsun jedi künlar künlari kibikj. hastalyg்ynyn murdar bolsun (Lev 12:2); eger jatma jatsa kiši anynbyla da bolsa qany j. ol qatynnyn ol kiši üstüńa (Lev 15:24); qatyn ki aqsa aqmag்y qanynyn köp künlar vahtyndan bašqa j. jemeśa ki aqsa juvuq vahtyna j. bar künlarind́a aqmaġynyn murdarlyg்ynyn künlari kibik j. bolsun murdardy ol (Lev 15:25); bar ol töśak ki \{jatsa\} anyn üstüna bar zavly künlarind́a töšagi kibik j. bolsun anar da bar ol savut ki oltursa anyn üstüńa murdar bolsun murdarlyg்y kibikj. (Lev 15:26)

e Mod.SWKar. jyhyslyk 'mentruation', Mod.NWKar. jyhyz 'impurity'.

jylan: küvdürgüč jylan 'viper, poisonous snake'

Dan jylan kibik jolqatyna k. j. kibik izqatyna ol tišlevčü soġančyqlaryndan atnyn da tüšedi atlanuvčusu artqary (Gen 49:17); ajtty Adonaj Mošeǵa qylg̀yn özüja k.j. da qojgun any alam üstüńa da bolġaj bar ol tišlanǵan jylandan da baqsyn anyn üstüńa da savugur (Num 21:8)

-larny: ijdi Adonaj ulusta ošol ol k. j. da tišladilar ošol ol ulusnu da öldü köp ulus Jisra’eld́an (Num 21:6)

e Mod.NWKar. kuv́dứǵuć ilan 'dragon', kuv́durǵguć 'burning', Mod.NWKar. jylan 'snake; viper'.

jyjyn '1. army; 2. military service'

otuz jaštan da joġarraq enli jašqadejin sanaġyn alarny bar ol kelüvčünü jyjynlama j. qulluq etŕa qulluq ohel mo'edd́a (Num 4:23); budur ol iš ki qylġyn Levililarǵa igirmi beš jaštan da joġarraq kelsin jyjytma j. qulluġunda ohel mo'ednin (Num 8:24)

-g̀a: otuz jaštan da joġarraq da enli jašqadejin bar ol kelüvčü j. qylma iš ohel mo'edd́a (Num 4:3); otuzjaštan dajoġarraq da enlijašqadejin sanag்yn alarny bar ol kelüvčünü j. qulluq etŕa ošol qullug்un ohel móednin (Num 4:30); otuz jaštan da jog்arraq da enli jašqadejin bar ol kelüvčünü j. qulluq etḿa ohel mo'edd́a (Num 4:35); otuz jaštan da joġarraq da enli jašqadejin bar ol kelüvčü j. qulluqqa ohel mo'edd́a (Num 4:39); otuz jaštan da joġarraq da enli jašqadejin bar ol kelüvčü j. qulluqqa ohel móedd́a (Num 4:43)

-yndan: enlijaštan qajtsyn j. ol qulluqnun da qulluq etḿasin artyq (Num 8:25)

e WKar. jyjyn 'crowd, gathering; group; host', Mod.EKar. yjyn '1. crowd, gathering; 2. army', cf. BHeb. צבד '1. military service; 2. host, fighting men'. $\Rightarrow$ jyjynla-

jyjynla- 'to join up'

-ma: otuz jaštan da joġarraq enli jašqadejin sanaġyn alarny bar ol kelüvčünü j. jyjyn qulluq etḿa qulluq ohel mo'edd́a (Num 4:23); budur ol iš ki qylg̀yn Levililarǵa igirmi beš jaštan da joġarraq kelsin j.jyjyn qulluġunda ohel mo'ednin (Num 8:24) 
e Mod.NWKar. jyjyn 'crowd, gathering; group; host' + deriv. suff., cf. Mod.EKar. yjyn '1. crowd, gathering; 2. army'. $\Rightarrow$ jyjyn

K

kajaccet- 'to repent'

-me: mode bolma da jüregïbyla k. jazyqly išlerï üčün (Deu 30:14)

e Pol. kajać się 'to repent, to confess repentantly'.

kaporet 'Kaporet (the cover of the Holy Ark)'

qylgyn k. aruv altyndan eki lokotlar uzunlug்u anyn da lokot dajarym kenligi anyn (Exo 25:17); bolsunlar ol keruvlar jajuvčular qanatlar jog்aryg்a quršavčular qanatlarybyla ol k. üstüne (Exo 25:20); da sözlermen birgeje ol k. üstünden (Exo 25:22); qyldy k. aruv altyndan (Exo 37:6); ediler ol keruvlar jajuvčular qanatlar jog்aryg்a quršavčular qanatlarybyla ol k. üstüne (Exo 37:9); passim

-ke: bolsunlar ol keruvlar jajuvčular qanatlar joġaryg்a quršavčular qanatlarybyla ol kaporet üstüne da jüzleri alarnyn kišinin qaryndašyna ol k. bolsunlar jüzleri ol keruvlarnyn (Exo 25:20); jüzleri alarnyn kiši qaryndašyna ol k. ediler jüzleri ol keruvlarnyn (Exo 37:9)

-lar: bolsunlar ol k. jajuvčular qanatlar jog்aryg்a quršavčular qanatlarybyla ol kaporet üstüne (Exo 25:20)

-ni: bergin ošol ol k. ol aron üstüne (Exo 25:21); bergin ošol ol k. aronu üstüne ol šarajatnyn (Exo 26:34); ošol ohel mo'edni da ošol ol aronnu šarajatqa da ošol ol k. ki anyn üstüne (Exo 31:7); ošol ol aronnu da ošol tutqučlaryn anyn ošol ol k. da ošol parohetni ol qaplavny (Exo 35:12); aronun ol šarajatnyn da ošol tutqučlaryn anyn da ošol ol k. (Exo 39:35); passim

-nin: bergin any alnynda ol parohetnin ki aronu üstüne ol šarajatnyn alnynda ol k. ki ol šarajat üstüne (Exo 30:6); qyldy eki keruvlar altyndan qaqqan qyldy alarny eki učlaryndan ol k. (Exo 37:7); kelḿasin har vahtta ol qodeška ičḱkrtin parohetḱka alnyna ol k. (Lev 16:2); bürksün barmag்y byla alnynda ol k. kün tuvušusary da alnynda ol k. bürksün jedi keŕatlar ol qandan barmaġy byla (Lev 16:14); bürksün any ol kaporet üstüna da alnynda ol k. (Lev 16:15); passim

-ten: keruv učtan bunjatyn da bir keruv učtan bu jantyn ol k. (Exo 37:8)

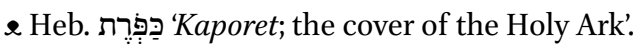

kapusta 'cabbage'

-larny: saġyndyq ošol ol balyqny ki ašaredik Micrida muft ošol ol hyjarlarny da ošol ol harbuzlarny da ošol ol k. da ošol ol soganlarny (Num 11:5)

\& Pol. kapusta 'cabbage', Russ. кanycma id., Ukr. кanycma id.

karbankulis (?), L (?) 'carbuncle'

-nin: dört jergeler taštan jergesin rubinnin smaraknyn da k. ol bir jerge (Exo 28:17); jergesi rubinnin šmaraknyn da k. (Exo 39:10) 
e Russ. arch. карбункулъ карвамиколосъ карвамуколосъ 'carbuncle', see SRJaXVI-XVII (VII: 77), cf. Pol. karbunkut id., Lat. carbunculus id.

keleš- 'to betroth'

-sej: qatyn k. da özge kiši alyr any üv qondarsaj da olturmassen anda borlalyq or\{na\}tsaj da a. čýgarmassen any (Deu 28:30)

-ti: qajsy ol kiši ki k. qatyn da almady (Deu 20:7)

e Mod.NWKar. kelašiń- 'to get engaded'.

\section{kelüv 'ascent'}

-üńa: quršar sizǵa ol ček tüšlük sartyn k. 'Aqrabimnin (Num 34:4)

e Mod.NWKar. Keluv' 'the coming'.

kemin: kemin künnün kününde kemin künnün kününd́a 'every day'

ol qystavčular žahtlaredilar ajtadog்ač tügellejiz išlerijizni k. k. kününde ki nečik bolganda ol bičen (Exo 5:13); eksitmejiz kirpičler[i]jizden k. k. kününde (Exo 5:19); da čygar ol ulus da čöplerler k. k. kününd́a anyn üčün ki synaġajmen any (Exo 16:4); bulardylar mo'edĺari Adonajnyn ki ündajiz alarny aziz ünd́almüšlar juvutma otlu qarban Adonajġa ola da tirki debeha da nesehlar k. k. kününd́a (Lev 23:37)

? Mod.EKar. kemin künniך küninde 'every day'.

\section{keruv 'cherub'}

bir k. učtan bu jantyn da bir k. učtan bu jantyn (Exo 25:19); bir k. učtan bunjatyn da bir k. učtan bu jantyn ol kaporetten qyldy ošol ol keruvlary eki učlaryndan (Exo $37: 8)$

-lar: qylgynn eki k. altyndan (Exo 25:18); bolsunlar ol k. jajuvčular qanatlar jog்aryg்a (Exo 25:20); qyrmyzy jipekten k. išin ojuvlavčunun (Exo 26:1); qyrmyzy jipekten k. išin ojuvlavčunun qyldy alarny (Exo 36:8); ešken bezden išin ojuvlavčunun qyldy any $k$. (Exo 36:35); qyldy eki k. altyndan qaqqan qyldy alarny (Exo 37:7); passim

-larny: qylyjyz ošol ol k. eki učlary üstüne anyn (Exo 25:19)

-larnyn: ol kaporetke bolsunlar jüzleri ol k. (Exo 25:20); arasyndan ol eki k. ki aronu üstüne (Exo 25:22); ol kaporetke ediler jüzleri ol k. (Exo 37:9); arasyna eki ol k. (Num $7: 89$ )

-lary: bir keruv učtan bunjatyn da bir keruv učtan bu jantyn ol kaporetten qyldy ošol ol k. eki učlaryndan (Exo 37:8)

e Heb. כּּרְבִ 'cherubim', Mod.NWKar. keruvim 'the cherubim'.

kiji|k 'wild animal'

da bar ol k. ら̌ynsysajyn da bar ol tuvar žynsysajyn (Gen 7:14); bar ol k. bar ol qomuz da bar ol quš (Gen 8:19); ajtyrbiz jaman k. ašady any (Gen 37:19); jaman k. ašady any jyrtylmajyrtyldy Josef (Gen 37:12); passim

-|gin: tuvarda qomuzda k. jernin (Gen 1:24); bar k. ol jernin da ošol bar qušun ol köklernin (Gen 2:19); ijarmen sizd́a ošol k. ol tüznün (Lev 26:22);

-|gind́an: ol jylan edi ustatraq bar [k.] tüznün (Gen 3:1); qarg்yšlyraqtyrsen bar ol tuvardan da bark. ol tüznün (Gen 3:14) 
-|gine: bolur gövdej jemge qušuna ol köklernin da k. ol jernin (Deu 28:26) $\Rightarrow$-gińa. -gińa: bar k. ol jernin da bar qušuna ol köklernin (Gen 1:30); qušuna ol köklernin da bark. ol tüznün (Gen 2:20)

-|gi: bar [k.] üstüńa ol jernin (Gen 9:1); bar k. byla ol jernin (Gen 9:1o); ašasyn k. ol tüznün (Exo 23:11); artar üstüje k. ol tüznün (Exo 23:29); mag்at artar üstüja k. ol tüznün (Deu 7:22); passim

-ka: tuvaryja da k. ki jerijd́a bolsun (Lev 25:7)

-lernin: da tišin jaman k. ijermen alarda (Deu 32:24)

-ni: saġyndy Tenri ošol Novaḥny da ošol bar ol k. (Gen 8:1); da eksitirmen jaman k. ol jerd́an (Lev 26:6)

-nin: bar k. izlerḿan any (Gen 9:5); gövd́asińa murdar k. (Lev 5:1)

-t́a: tyndy bar ten ol tebrenüvčü ol jer üstüńa qušta da tuvarda da k. da bar ol qomuzda ol qozlajdoġan ol jer üstüńa da bar ol adam (Gen 7:21)

e Mod.NWKar. kijik 'wild'.

kippi 'seed, pip'

-ǵa: baryndan neki qylyndy borlalyqtan ol vina qabuqlardan da k. dejin ašamasyn (Num 6:4)

e Mod.SWKar. kippi 'seed, pip'.

kipurim 'atonement'

qanyndan hatatynyn ol k. kününün bir keret jylda (Exo 30:10); künü ol k. ol aziz ünd́almüš bolsun sizǵa (Lev 23:27); ašyrġyn qyčqyrmaqbyla šofar avazy ol jedinči janġajda onunču kününd́a janġajnyn ol k. kününd́a ašyryjyz šofar avazy bar jerijizd́a (Lev 25:9)

-nin: heč iš qylmajyz kensisind́a ošpu künnün ki künüdü ol k. ol bošatlyq qolma siznin üčün alnynda Adonaj Tenrijiznin (Lev 23:28); hatatyndan olk. (Num 29:11)

e Heb. כִּפְּרִ 'atonement'.

\section{kohen gadol 'Hight Priest'}

jedi künler kijsin alarny ol kohen qajsy bolsa k. g. (Exo 29:30); eger ol jaġyndyrylġan kohen ki ol k. g. jazyqly bolsa (Lev 4:3); ol kohen ol jagyyndyrylgan ki oldu ol k. g. ki bolsa Aharon ornuna uvullaryndan anyn (Lev 6:15); ol k. g. qatynny bojluqlarybyla alsyn (Lev 21:13); ol k. g. ki jaġyndyrdy any ol aziz javbyla (Num 35:15); ki šaharynda miqlatynyn oltursun ölgünća ol k. g. da ölüm sortun ol k. g. qajtsyn ol qaraqčy jerińa tutuvluġunun (Num 35:28); almajyz juluv andijd́an kimǵa kéraklidi qačma šaharyna miqlatynyn qatma olturma jerd́a ölgünća ol k. g. (Num 35:32)

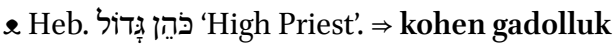

kohen gadolluk 'high-priesthood'

-qa: aziz upraqlar ki Aharonnun bolsunlar uvullaryna özünden sortun jag்ynma alarbyla k. g. da tolturma alarbyla ošol qolun alarnyn (Exo 29:29); jaġyndyrylgan kününd́a k. g. (Lev 6:13)

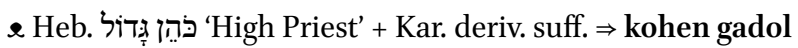




\section{koryta 'gutter'}

-larda: turg்uzdu ošol ol tajaqlarny ki jondu k. suvlavlarynda ol suvlarnyn (Gen 30:38); qojaredi Ja'aqov ošol ol tajaqlarny közlerića ol qojnun k. issitḿa alarny tajaqlar byla (Gen 30:41)

-larny: tolturdular ošol ol k. ičirḿa ošol qojun (Exo 2:16)

e Pol. koryto 'gutter', Russ. корыто id., Ukr. корыто id.

kos 'little owl'

-nu: ošol ol k. da ošol ol šalahny da ošol ol janšufnu (Lev 11:17); ošol ol k. da ošol ol janšufnu da ol tinšemetni (Deu 14:16)

\& Heb. סiּ ‘a kind of owl', see Klein (1987: 273), 'little owl', see Koehler \& Baumgartner (1985: 428). In the quoted context, the word should not be linked with Mod.SWKar. kos 'thrush (bird)' < Pol. kos 'blackbird'.

koš 'basket'

-un: alġyn bir k. da bergin ary tolu omer ündeledoġan ölčüvnü man (Exo 16:33)

\& Pol. kosz 'basket'.

kovah 'a kind of lizard; monitor lizard (?), spotted lizard (?)'

ol anaqa da ol k. da ol leta'a da ol homet da ol tinšamet (Lev 11:30)

\& Heb. כָָּ 'a kind of a lizard', see Klein (1987: 274), Koehler \& Baumgartner (1985: 430).

köbüsü 'many'

ajtty 'Esav bardy mend́a k. qaryndašym bolsun saja neki senin (Gen 32:10); edi mallary alarnyn k. olturmaqtan birǵa (Gen 36:7); jyštyrdy Josef bürtük qumun kibik ol tengiznin k. astry neginče ki qaldy sanamaqtan kijoġedi san (Gen 41:49); dag்yn qatyš ol k. bardy birgelerińa alarnyn (Exo 12:38); maġat buzarlar ol čekni alnynda Adonajnyn baqma da tüšer andan k. (Exo 19:21); k. tuvar edi ulanlaryna Ru'uvennin (Num 32:1)

e Mod.SWKar. kebisi 'many'.

köčür- 'to move, to transport'

-dü: k. andan čatyryn ol tavġa mizrạ̣ sartyn Bet'elge (Gen 12:8); k. čatyryn andan da qazdyözǵa quju (Gen 26:22); k. Moše ošol Jisra’elniJam Suftan (Exo 15:22)

-makḱa: qylg̀yn özüja eki byrg்ylar ... da bolsunlar saja ünd́amakḱa ol žymatny da k. ol avullarny (Num 10:2)

e Mod.NWKar. Koč́ur- 'to translate', Mod.EKar. köčir- 'to move, to transport'.

\section{kögöt 'plant'}

da berirmen k. tüzüjde (Deu 11:15); da ösmesti anda heč k. (Deu 29:22)

-nü: da ajtty Tenri muna berdim sizǵa ošol bar k. urluqlavčunu urluq ki jüzlari üstüńa bar ol jernin (Gen 1:29)

-nün: bar ješilligin k. jemǵa (Gen 1:30); bar qomuz ki ol tiridi sizǵa bolsun [jem] ǵa ješilliginkibik k. (Gen 9:3)

-ü: da bar k. ol jernin (Gen 2:5); bar k. üstüne ol tüznün jerinde Micrinin (Exo 9:21) 
-ün: da ašarsen ošol k. ol tüznün (Gen 3:18); da ošol bar k. ol tüznün vatty ol buz (Exo 9:24); da ašady ošol bark. ol jernin (Exo 10:15)

-ünd́a: da qalmady heč ješillik ag̉ačta da k. ol tüznün (Exo 10:15)

e Mod.NWKar. koǵot 'grass'. $\Rightarrow$ kögüt

kögüt 'plant'

-ün: sunġun qolujnu jeri üstüne Micrinin čegirtke byla da kelsin ... da ašasyn ošol bar k. ol jernin (Exo 10:12)

e Mod.NWKar. koǵot́ 'grass'. $\Rightarrow$ kögöt

kölendra 'coriander'

-nyn: atadylar üvü Jisra’elnin ošol atyn anyn man da ol edi urlug̉u kibik k. (Exo 16:31)

e Mod.NWKar. kolendra 'coriander', Pol. kolendra id. $\Rightarrow$ köĺandra

kölandra 'coriander'

-nin: ol man edi urluǵu kibik k. (Num 11:7)

\& Mod.NWKar. kolendra 'coriander', Pol. kolendra id. $\Rightarrow$ kölendra

könü 'free'

eger klemeśa ol qatyn barma artyjdan da k. bolursen antymdan bu tek ošol uvlumnu qajtarmag̉yn ary (Gen 24:8); ol vahtta k. bolursen qarg்yšymdan ki kelśaj uruvuma da eger bermeselar saja da bolursen k. qarg்yšymdan (Gen 28:41); ki alsa kiši janġy qatyn čyqmasyn čerüvge da ašmasyn anyn ašyra heč nemede $k$. bolsun üvünde bir jyl da bijendirsin ošol qatynyn ki aldy (Deu 24:5)

-ler: ol kim ki tabulsa birgesina ol bolur maja qul da siz bolursiz k. (Gen 44:10) $\Rightarrow$-lar -lar: andan sortun qajtyrsiz da bolursiz $k$. Adonajdan da Jisra'eld́an (Num 32:22) $\Rightarrow$-ler

e Mod.NWKar. końu 'innocent'.

kötür-: ajaqlar kötür- *'to set off'

-dü: k. Ja'aqov ajaqlaryn da bardy jerińa Qedemnin (Gen 29:1)

e Mod.NWKar. ajah 'leg', Mod.NWKar. kotur- 'to carry'.

krolik 'rabbit'

-ni: ošol ol k. hot́a güvün keltiŕadi eśad́a ol da tujaq tujaqlamajdy murdardy ol sizǵa (Lev 11:5); ošolol k. hote güvün keltiredilerde ese alar da tujaq tujaqlamajdylarmurdarlardylar alar sizge (Deu 14:7)

e Ross. кролик 'rabbit'.

kruh '*ledge; rim'

-u: bergin any k. tübüne olmizbeahnnyn ašaġartyn (Exo 27:5); qyldy mizbeahqqa elek iši avnyn tučtan k. tübüne anyn ašag்artyn jarymyndadejin (Exo 38:4)

\& Ukr. круг 'cirlce; round object', cf. Mod.NWKar. kruḥ 'circle'.

küč 'hand; the hand of God'

-ü: ajtty ki nečik bolsa k. bijliknin taḥty üstüne bijliginin Jisra’elnin (Exo 17:16)

-üj: on k. senin e Adonaj küčlüdü quvatbyla on hyššymyj senin e Adonaj syndyrdy dušmanny (Exo 15:6)

e Mod.NWKar. kuć 'strength, power', cf. Mod.NWKar. kudrat '1. power; 2. the right 
hand of God', Mod.NWKar. kuvat '1. strength, power; 2. the hand of God', Heb. יִמינ:ּד '1. power; 2. the right hand of God'.

küjöv 'son-in-law'

-lerinin: čygyjyz ol orundan ošpu ki čejpajdy Adonaj [o]šol ol šaharny da edi kültkü külüvčü kibik közlerind́a k. (Gen 19:14)

-lerińa: čyqty Lot da sözledi k. aluvčularġa qyzlaryn da ajtty turujuz čyg்yjyz ol orundan (Gen 19:14)

-nü: ajttylar ol erenler Lotqa dag்yn kim bardy saja bunda k. da uvullaryjny da qyzlaryjny (Gen 19:12)

e Mod.NWKar. kijov 'son-in-law'.

künd́aš *'rival wife' (?)

qatynny tuvdug் qatyna almag்yn k. etŕa aškartma ajipin anyn anyn qatyna tirliklarind́a anyn (Lev 18:18)

e MMod.NWKar. kun 'day' + deriv. suff. that forms nouns denoting possessors of a shared attribute, i.e. kündaš means 'the one who shares the day (with someone)'.

künülamak 'jealousy'

-nin: tašsa anyn üstüna sajgy̌̌y k. da künülaśa qatynyna da ol murdar bolundu jemeśa ašsa anyn ašyra saġyšy $k$. da ol murdar bolunmady (Num 5:14); jemeśa kiši ki ašsa anyn üstüńa saġyš k. (Num 5:30)

-larnin: levona ki tirkisidi k. (Num 5:15); alsyn ol kohen qolundan ol qatynnyn ošol tirkisin ol k. da sunsun ošol ol tirkini alnynda Adonajnyn (Num 5:25); budur üvratüvü ol k. ki azsa qatyn boladoġač erki tübüńa erinin (Num 5:29)

e Mod.NWKar. Kuńula- 'to envy', Mod.SWKar. kinilemek 'jealousy, envy'.

küstün- 'to sigh'

-dülar: öldü biji Micrinin da k. uvullary Jisra’elnin ol qulluqtan da firjat ettilar da kötürüldü firjatlary alarnyn alnyna ol Tenrinin ol qulluqtan (Exo 2:23)

e Mod.NWKar. Kostun- (!) 'to sigh', Mod.EKar. küstün- 'to sigh, to groan'.

küvḿa|k *'sore, inflamation' (?)

adam ki bolsa terisind́a gufunun k. jemeśa šišik jemeśa temgil (Lev 13:20)

-|gi: baqsyn any ol kohen da muna k. ol hastalyqnyn aq qyzyl syman artynda bašynyn (Lev 13:43)

-|gidi: eger ornunda tursa ol temgil jajylmady terid́a da ol tundu k. ol küvmiaknin ol da aruv etsin any ol kohen qyzuvudu ol küvmiaknin ol (Lev 13:28)

-nin: eger ornunda tursa ol temgil jajylmady terid́a da ol tundu küvmagidi ol k. ol da aruv etsin any ol kohen qyzuvudu ol k. (Lev 13:28); k. da šišiknin da temgilnin (Lev 14:56)

e Mod.NWKar. kuv́mak 'burning'. 
L

lešem 'jacinth'

ol üčünčü jerge l. ševo da aḥlama (Exo 28:19); ol üčünčü jerge l. ševo da ahlama (Exo 39:12)

\& Heb. לֶׁשֶ 'a precious stone, usually identified with the opal or with the jacinth'.

leta'a 'lizard (?)'

ol anaqa da ol kovaḥ da ol l. da ol homet da ol tinšamet (Lev 11:30)

\& Heb. לִָָָָּ 'lizard', see Klein (1987: 299), 'gecko', see Koehler \& Baumgartner (1985: $480)$.

levona 'frankincense'

ajtty Adonaj Mošege alġyn özüje otjamlar mušk da revent da galban otjamlar da aruv l. bašqa bašqa har birisi bolsun (Exo 30:34); da bersin anyn üstüna l. (Lev 2:1); bergin anyn üstüńa jav da qojgiun anyn üstüńa l. tirkidi ol (Lev 2:15); berḿasin anyn üstüńa l. ki hatatty ol (Lev 5:11); bergin ol tüzüv üstüna aruv l. (Lev 24:7); bermasin anyn üstüńa l. ki tirkisidi künülamaklarnin (Num 5:15)

-ny: da ošol bar ol l. ki ol tirki üstüńa da tütatsin ol mizbeạ̣ta (Lev 6:8)

-sy: ajyrsyn andan tolu uvuču öźagind́an da javyndan anyn bar l. byla da tutatsin ol kohen ošol tütüsün anyn (Lev 2:2); bar l. byla otlu qarban Adonajga (Lev 2:16)

\& Heb. לִבְֹ 'frankincense'.

$\log$ 'log (a liquid measure)'

üč esronlar öźak tirki jumurulġan javbyla da bir l. jav (Lev 14:10); ošol l. ol javny (Lev 14:12); alsyn ol kohen l. ol javdan (Lev 14:15); öźak jumurulgan javbyla tirkiǵa da bir l. jav (Lev 14:21); alsyn ol kohen ošol qozusun ol fašmanlyq qarbannyn da ošol l. ol javny (Lev 14:24)

e Heb. לג' 'log; a liquid measure'.

loś 'moose'

-nu: sog்ag்ny da jürnü da bojvolnu da pole qojun da dišonnu da l. da žubrany (Deu 14:5)

e Mod.SWKar. los 'moose', Pol. toś 'moose', Russ. лось id., Ukr. лось id.

$\mathbf{M}$

ma'arav 'west'

Bet’el m. sartyn (Gen 12:8); ki sen anda cafon sary da tüšlük sary da mizraḥ sary da m. sary (Gen 13:14); da jajylyrsen m. sary da kün tuvušu sary da cafon sary da tüšlüksary (Gen 28:14); čüvürdü Adonaj m. jeli küčlü astry (Exo 10:19); qyryjlaryna ol miškannyn m. sary qylgyn alty tahtalar (Exo 26:22); passim

-ny: da Naftali üčün ajtty Naftali tojġan [kl]ekten da tolu alġyšyndan Adonajnyn m. da daromnu meresle (Deu 33:23) 
-nyn: kenligi ol qahranyn tarafyna m. enler enli lokot (Exo 27:12); tarafyna m. enler enli lokot baġanalary (Exo 38:12); čegim. da bolur sizǵa ol ullu tengiz da ček bu bolur sizǵa čegi m. (Num 34:6); da ošol tarafyn m. eki min lokotbyla (Num 35:5);

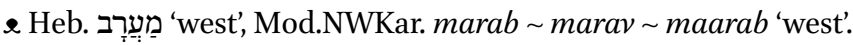

maca 'unleavened bread'

eger tirki išlanǵan ol tava üstüna bolsa qarbanyj öźak jumurulġan javbyla $m$. bolsun (Lev 2:5); četanindan ol macalarnyn ki alnynda Adonajnyn aldy hala m. bir da hala ötḿak javly bir (Lev 8:26); alsyn ol kohen ošol ol bilakni bišḱkanni ol qočqardan da hala m. bir ol četand́an da juġa m. bir da bersin uvučlary üstüńa ol nazirnin (Num 6:19)

-lar: ašasynlar ošol ol etni ošpu kečede šišlege[n]ni otta da m. marorlar byla ašasynlar any (Exo 12:8); jedi künler m. ašajyz (Exo 12:15); ašajyz m. ol egirmi biriniči kününedejin janġajnyn (Exo 12:18); heč quక̌urajġanny ašamajyz bar olturušlaryjyzda ašajyz m. (Exo 12:20); biširdilar ošol ol hamurnu ki čyġardylar Micridanjajmalar m. ki quక̌urajmaġan edi (Exo 12:39); jedi künler ašag்yn m. da ol jedinči künde hyž Adonajg̉a (Exo 13:6); passim

-larny: saqlajyz ošol ol m. (Exo 12:17)

-larnyn: ošol hyy̌yn ol m. saqlag்yn jedi künler ašag்yn macalar ki nečik bujurdum saja (Exo 23:15); boḥon ötmek bir da hala ötmek javly bir da joġa bir četeninden ol m. ki alnynda Adonajnyn (Exo 29:23); ošol hyy̌̌yn ol m. saqlaġyn jedi künler ašag்yn macalar (Exo 34:18); da ošol četanin olm. (Lev 8:2); četanind́an olm. ki alnynda Adonajnyn aldy hala maca bir da hala ötmak javly bir (Lev 8:26); passim

\& Heb. מַָּ 'unleavened bread'.

maca: hyžy ol macalarnyn 'Passover, the Feast of Unleavened Bread'

onbešinči kününd́a ošpu janġajnyn ḥ. o. m. Adonajg̉a jedi künlar macalar ašajyz (Lev 23:6)

-n: ošol hyžyn o. m. saqlag̉yn jedi künler ašag்yn macalar ki nečik bujurdum saja vaġdasynda ol baš qusqan janġajnyn ki ol vahtta čyqtyj Micriden da körünmesinler qyblalarym alnyna boš (Exo 23:15); ošol hyy̌̌yn o. m. saqlag̉yn jedi künler ašag்yn macalar ki bujurdum saja (Exo 34:18)

-nda: ne orunda ki sajlasa h̆y̌žnda o. m. (Deu 16:16)

\& Heb. מַצָ 'unleavened bread'.

maceva '1. pillar; 2 . tombstone'

da aldy ošol ol tašny ki qojdu bašlary tübüna da qojdu any m. da qujdu jav bašyüstüna anyn (Gen 28:18); ol taš ošpu ki qojdum m. bolur üvü ornuna Tenrinin (Gen 28:22); menmen ol Tenri Tenrisi Bet'elnin ki jaġyndyrdyj anda m. ki nijetettij maja anda nijet (Gen 31:13); aldy Ja'aqov taš da kötürdü any bolma m. (Gen 31:45); ajtty Lavan Ja'aqovga muna ol oba ošpu da muna ol m. ki saldym arama da araja (Gen 31:51); tanyqty ol oba ošpu da tanyqty ol m. eger men dušman bolsam saja ki ašmajajmen saja ošol ol obany ošpunu (Gen 31:52); turġuzduJa'aqov m. ol orunda ki sözledi birgesine tašly m. da q[u] jdu anyn üstüńa neseh da q[u] jdu anyn üstüńa jav (Gen 35:14); 
turg்uzdu Ja'aqov m. zereti üstüńa anyn oldu macevasy zeretinin Rahelnin ol künge dejin (Gen 35:20); passim

-laryn: ki buzma buzg்un alarny da syndyrma syndyrg்yn m. alarnyn (Exo 23:24); ošol mizbeahlaryn alarnyn qavšatyjyz da ošol m. alarnyn syndyryjyz (Exo 34:13); bulaj qylyjyz alarga mizbeaḥlaryn alarnyn qavšatyjyz da m. alarnyn syndyryjyz (Deu 7:5); qavšatyjyz ošol mizbeaḥlaryn alarnyn da syndyryjyz m. alarnyn (Deu 12:3)

-ny: da eger sen dušman bolsaj maja ki ašmaġajsen maja ošol ol obany ošpunu da ošol ol m. ošpunu jamanlyqqa (Gen 31:52)

-sy: turg்uzdu Ja'aqov maceva zereti üstüna anyn oldu m. zeretinin Rahelnin ol künge dejin (Gen 35:20)

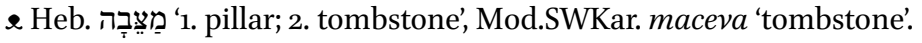

mažunlavču 'apothecary'

-nun: maร̌un mažunlanġan iši m.jag்ynmaq jav aziz bolsun (Exо 30:25); qylg்yn andan tütü mažun iši m. qošulgan aruv aziz (Exo 30:35); aruv iši m. (Exo 37:28)

\& Mod.SWKar. mazunlavcu 'apothecary'.

mamzer 'bastard'

kelmesin m. qahalyna Adonajnyn (Deu 23:3)

^ Heb. מַמְזָר 'bastard'

maror 'bitter herb'

-lar: ašasynlar ošol ol etni ošpu kečede šišlege [n] ni otta da macalar m. byla ašasynlar any (Exo 12:8)

-larbyla: qylsynlar any macalar byla da m. ašasynlar any (Num 9:11)

\& Heb. מָרוֹ 'bitter herbs'

maskim: maskim bol- 'to consent'

-ma: klemegin m. b. jeldemegine anyn (Deu 13:9)

e Heb. מַסְכִּים 'consent' + Kar. aux. verb.

mašijạ̣ 'Messiah'

neginče ki kelǵaj m. da anar bolur tynlamaġy uluslarnyn (Gen 49:10)

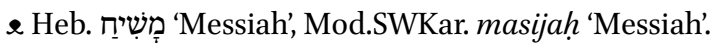

micnefet 'turban'

bulardylar ol upraqlar ki qylsynlar hošen da efod da qaftan da ojuvlu kölmek m. da avnet da qylsynlar aziz upraqlar Aharonġa qaryndašyja da uvullaryna anyn kohenlik etme maja (Exo 28:4); qojg்un any kök šnur üstüne da bolsun ol m. üstüne (Exo 28:37); bergin ošol ol altyn tačny ol m. üstüne (Exo 29:6); berdiler anyn üstüne kök šnur berme ol m. üstüne joġartyn (Exo 39:31); qojdu ol m. üstüna uturusuna jüzlarinin ošol čalmasyn ol altyn aziz tačnyn (Lev 8:9)

-ni: qojg்un ol m. bašyüstune anyn (Exo 29:6); ošol ol m. bezden da ošol ol bijik börklerni bezden (Exo 39:28); qojdu ošol ol m. bašy üstüńa anyn (Lev 8:9)

-nin: uturusuna ol m. bolsun (Exo 28:37)

e Heb. מִצְנֶפֶּ 'turban'. 


\section{Micrajim 'Egypt'}

-d́an: Hevron jedijyllar qondaryldy burunraq Cóan M. (Num 13:22)

e Heb. מִצְרַיִ 'Egypt'.

mihnesajim 'dual trausers'

-ler: qylg̀yn alarg்a aq m. qaplama etin ajipnin bellerden da butlarg்adejin bolsunlar (Exo 28:42) $\Rightarrow$-ĺar

-lerni: ošol ol micnefetni bezden da ošol ol bijik börklerni bezden da ošol ol m. ešken bezden (Exo 39:28)

-lar: kijsin ol kohen aq uprag்yn da aq m. bolsunlar gufu üstüna (Lev 6:3); aq kölmak azizni kijsin da aq m. bolsunlar gufu üstüńa (Lev 16:4) $\Rightarrow$-ler

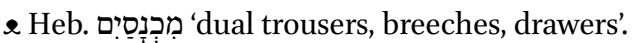

milu'im' 'to be set'

m. tašlary hošenge (Exo 25:7); šoham tašlary efodġa da m. tašlary ḥošenge (Exo 35:9); keltirdiler ošol ol šoham tašlaryn efodg்a da ošol ol m. tašlaryn hošenge (Exo 35:27)

\& Heb. מִלִִאים 'to be set'. $\Rightarrow$ milu'im'II

milu'im ${ }^{\text {II }}$ 'of ordination'

-dirĺar: aldy Moše \{alarny\} uvučlary üstünd́an alarnyn da tütatti ol mizbeahta ol 'ola üstüńa m. alar (Lev 8:28)

-ǵa: budur ol üvŕatüv olag்a da tirkiǵa da ḥatatqa da ašamg்a da m. da debeḥasyna ol šelamimnin (LEv 7:37)

-ĺarijiznin: ešigind́an ohel mo`ednin čyqmajyz jedi künlar tolġan künǵadejin künlari $m$. (Lev 8:33)

-nin: ki qočqarydym. ol (Exo 29:22); alġyn ošol ol töšnüqočqaryndan olm. (Exo 29:26); da ki ajyryldy qočqaryndan ol m. (Exo 29:27); ošol qočqaryn ol m. alġyn da biširgin ošol etin anyn (Exo 29:31); eger qalsa etinden ol m. (Exo 29:34); passim

\& Heb. מִלְלִאים of ordination'. $\Rightarrow$ milu'im ${ }^{I}$

miqlat 'refuge'

qondaryjyz özüjüzǵa šaharlar m. šaharlary bolsunlar sizǵa (Num 35:11); ošol ol üč šaharlary berijiz jerind́a Kena'annyn m. šaharlary bolsunlar (Num 35:14)

-qa: bolsunlar sizǵa ol šaharlar m. (Num 35:12); ulanlaryna Jisra’elnin da ġaripǵa da očarg்a ortalarynda alarnyn bolsunlar alty ol šaharlar ošpular m. qačma ary (Num $35: 15)$

-nyn: ošol ol šaharlar ki berijiz Levililarǵa alty šaharlaryn olm. (Num 35:6); ol šaharlar ki berśajiz alty šaharlary m. bolsunlar sizǵa (Num 35:13)

-ynyn: qajtarsynlar any ol క̌ymat šaharyna m. ki qačty ary (Num 35:25); eger čyqma čyqsa ol qaraqčy čegind́an šaharynyn m. ki qačty ary (Num 35:26); tapsa any gečeǵasi ol qannyn tyšqartyn čegińa šaharynyn m. (Num 35:27); šaharynda m. oltursun (Num 35:28); qačma šaharyna m. (Num 35:32)

e Heb. מִקְְָ 'refuge'. 


\section{mispahat 'scab'}

aruv etsin any ol kohen m. ünd́aĺadog்an hastalyqtyrol da juvsun upraqlaryn da arynyr (Lev 13:6); eger jajylma jajylsa ol m. terid́a körünǵanindan sortun ol kohenǵa arynganyndan sortun da körünsün ekinči kerat ol kohenǵa (Lev 13:7); körsün ol kohen da muna jajyldy ol m. terida da murdar etsin any ol kohen cara'attyr ol (Lev 13:8)

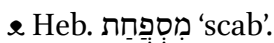

\section{miškan 'tabernacle'}

bolsun ol m. bir (Exo 26:6); qylg̀yn enler junundan ečkilernin čatyrg்a olm. üstüne (Exo 26:7); qyldy enli eslar altyndan da qoštu ošol ol enlerni birin birisine aslar byla da boldu ol m. bir (Exo 36:13); qyldy enler junundan ečkilernin čatyrg்a ol m. üstüne on bir enler qyldy alarny (Exo 36:14); passim

-da: da šehinasy Adonajnyn toldu ol m. (Exo 40:35)

-gaa: qylg̀yn ošol ol tahtalarny m. šitim ag்ačlaryndan turadog்anlarny (Exo 26:15); qylgyn ošol ol tậtalarny m. egirmi tạ̣ta (Exo 26:18); qyldy ošol ol tạ̣talarny m. (Exo 36:20); qyldy ošol ol tahtalarny m. (Exo 36:23); bar ol qazyqlar m. da qahrag்a čüvre tučtan (Exo 38:20); passim

-ny: ošol ol m. qylg்yn on enler ešken bezden (Exo 26:1); turg்uzg்un ošol ol m. (Exo 26:30); ošol ol m. da ošol čatyryn anyn (Exo 35:11); qyldylar bar uslu jürekli qyluvčularda ol išni ošol ol m. (Exo 36:8); keltirdiler ošol m. Mošege (Exo 39:33); passim

-nyn: barča nečik ki men körgüzemen saja ošol türsünün ol m. da ošol türsünün bar savutlarynyn da alaj qylyjyz (Exo 25:9); artyg்ačny ol artadog்anny enlerinde ol čatyrnyn jarymyn ol ennin ol artadoganny salgynn artyüstüne ol m. (Exo 26:12); bolsun salynadog்an janlary üstüne ol m. bu jantyn da bujantyn qaplama any (Exo 26:13); alaj qylgyn bar tahtalaryna ol m. (Exo 26:17); eki janyna ol m. tarafyna cafonnun egirmi tahta (Exo 26:20); passim

-y: sen symarlaġyn ošol ol Levililarni m. qatyna ol šarajatnyn (Num 1:50); kötürüldü ol bulut m. üstünd́an ol šarajatnyn (Num 10:11)

-ymny: murdar etḱkanlarind́a ošol m. (Lev 15:31)

-yn: turg்uzg்un ošol m. ohel mo'ednin (Exo 40:2); bar ol tijüvčü ölüǵa žanyna ol adamnyn ki ölśa da bürkünḿaśa ošol m. Adonajnyn murdar etti (Num 19:13)

-yna: bar ol juvuvču ol juvuq kelüvčü m. Adonajnyn öladi (Num 17:28)

-ynyn: bulardylar vakillikleri ol miškannyn m. ol šarajatnyn (Exo 38:21); tügendi bar qullugu m. ohel mo'ednin (Exo 39:32); alnynda m. Adonajnyn (Lev 17:4); saqlasynlar ol Levililar ošol saqlaryn m. ol šarajatnyn (Num 1:53); saqlavčularġa saqlavy m. Adonajnyn (Num 31:30); passim

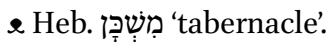

mišne 'copy'

jazsyn özüne ošol m. ol Torany ošpunu (Deu 17:18)

e Heb. מִשְׁנֶ 'copy'. 
mitqal 'a unit of weight; mithqal'

tynlaġyn meni jer dört jüz m. kümüšlük arama da araja nedir ol (Gen 23:15); ölćadi Avraham 'Efronġa ošol ol kümüšnü ki sözladi qulaqlaryča ulanlarynyn Hetnin dört jüz m. kümüš ašadog்an bezirgenǵa (Gen 23:16); bunu bersinler bar ol ašuvču ol sanalganlar qatyna jarymyn ol mitqalnyn mitqaly byla ol qodešnin egirmi gögdü ol $\mathrm{m}$. jarymyn ol mitqalnyn teruma Adonajga (Exo 30:13); beš jüz ol aziz m. byla da zejtun javy hin (Exo 30:24); ol tenufanyn egirmi tog்u qantar da jedi da otuz m. mitqaly byla ol qodešnin (Exo 38:24); passim

-lar:eger qulnu süzse olögüzjemese qaravašny kümüš otuz m. bersin bijine (Exo 21:32); keltirsin [...] ol qojdan qyjasyjbyla kümüš m. mitqaly byla ol qodešnin (Lev 5:15); da bolsun qyjasyj ol erkak igirmi m. da qatyn kišídan on m. (Lev 27:50); da bolsun qyjasyj ol erkak beš m. kümüš da qatyn kišidan üč m. kümüš (Lev 27:6); da bolsun qyjasyj on beš mitqal da qatyn kišidan on m. (Lev 27:7); passim

-nyn: bunu bersinler bar ol ašuvču ol sanalganlar qatyna jarymyn ol m. mitqaly byla ol qodešnin egirmi gögdü ol mitqal jarymyn ol m. teruma Adonajg̈a (Exo 30:13); ol hoža artyq bermesin da ol jarly azraq bermesin jarymyndan ol m. berme ošol terumasyn Adonajnyn (Exo 30:15); gög baštan jarymy ol m. mitqaly byla ol qodešnin (Exo $38: 26)$

-y: bunu bersinler bar ol ašuvču ol sanalġanlar qatyna jarymyn ol mitqalnyn m. byla ol qodešnin egirmi gögdü ol mitqal jarymyn ol mitqalnyn teruma Adonajġa (Exo 30:13); ol tenufanyn egirmi tog்uz qantar da jedi da otuz mitqal m. byla ol qodešnin (Exo 38:24); jüz qantar da min da jedi jüz da jetmiš beš mitqal m. byla ol qodešnin (Exo 38: 25); gög baštan jarymy ol mitqalnyn m. byla ol qodešnin (Exo 38:26); pas$\operatorname{sim}$

e Ar. مثقال mithqāl 'a unit of mass mostly used for measuring precious metals', cf. Mod.NWKar. mitkal 'shekel', Mod.SWKar. mitkal id.

\section{mohor 'signet'}

kimnindi ol m. da ol belibav (Gen 38:25)

-nun: išin us tasynyn tašnyn tužurmaqlaryn m. tužurg்un ošoleki ol tašlarnyatlarybyla uvullarynyn Jisra’elnin (Exo 28:22); qylg̀yn čalma aruv altyndan da tǔ̌urg்un anyn üstüne tǔ̌urmaqlary m. qodešla-H (Exo 28:36)

\& Mod.NWKar. mohor 'seal'.

\section{mohur 'signet'}

-nun: qyldylar ošol ol šoham tašlaryn quršalġanlar altyn ojuvlar byla tužurulġanlar tužurmaqlaryn m. atlary üstünne uvullarynyn Jisra’elnin (Exo 39:6); ol tašlar ediler atlary üstüne uvullarynyn Jisra'elnin alar ol on eki atlarybyla tužurmaqlary $m$. kiši atybyla ol eki ševetsajyn (Exo 39:14); da jazdylar anyn üstüne jazyšyn tužurmaqlarynyn m. קדָש לַיהוָֹ (Exo 39:30)

-uj: ajtty Tamar m. da belibavyj da tajaġyj ki qolujda (Gen 38:18)

e Mod.NWKar. mohor 'seal'. 
murdar et- 'to pronounce unclean, to declare unclean'

-ma: m. e. murdar etsin any ol kohen (Lev 13:44); aruv etía any jemeśa m. e. any (Lev 13:59)

-sin: baqsyn any ol kohen da m. e. any (Lev 13:3); da m. e. any ol kohen (Lev 13:8); da m. e. any olkohen (Lev 3:11); baqsyn ol kohen ošol čyj etni da m. e. any (Lev 3:15); da m. e. any ol kohen (Lev 3:20); passim

e Mod.NWKar. murdar et- 'to desacrate, to defile'.

musaf 'addition, additional thing'

-lary: bulardylar ol m. ki qylyjyz Adonajga mo'edlarijizd́a bašqa nijetĺarijizd́an (Num 29:39)

\& Heb. מוּסָר '1. addition; 2. additional prayer'.

müžešli: jymšaq müžešli *'with a soft sense of touch; tender' (?)

j. m. kiši sende da ol ašajyš astry jaman bag்ar közü anyn qaryndašyüstüne da qojundag்y qatyny üstüne da qaldyq ulanlary üstüne ki qaldyrsa (Deu 28:54); ol j. m. qatyn send́a da ol ašajyš ki čynyqmady tabany ajaġynyn turma ol jer üstüne ašajyšlyqtan da jymšaq müžešlikten jaman bag்ar közü anyn qojundag் eri üstüne da uvlu üstüne da qyzy üstüne (Deu 28:56)

e Mod.NWKar. jymšaḥ 'soft', Ar. مجس mağass 'spot which one touches or feels; sense of touch' or (Ar. >) Pers. مجس mağass 'the pulse or any part felt' + Kar. deriv. suff., cf. SWKar. minzesli in H (Deu 28:54, Deu 28:56) and NWKar. minžešli in K (Deu 28:54). $\Rightarrow$ müčešlik

müžešlik: jymšaq müžešlik *'teenderness, sensitivity'

-ten: ol jymšaq müžešli qatyn send́a da ol ašajyš ki čynyqmady tabany ajag்ynyn turma ol jer üstüne ašajyšlyqtan daj. m.jaman baġar közü anyn qojundag்y eri üstüne da uvlu üstüne da qyzy üstüne (Deu 28:56)

e Mod.NWKar. jymšaḥ 'soft', Ar. بجس mağass 'spot which one touches or feels; sense of touch' or (Ar. >) Pers. بجس mağass 'the pulse or any part felt' + Kar. deriv. suff., cf. SWKar. minzeslik in H (Deu 28:56). $\Rightarrow$ müčešli

N

nahar 'Euphrates'

-dan: öldü Samla da bij boldu anyn ornuna Ša’ul Rohovot ol n. (Gen 36:37)

e Heb. (Aram.) נָזָר 'river'.

nasi 'prince'

ki n. jazyqly bolsa da qylsa birni bar micvalaryndan Adonajnyn Tenrisinin (Lev 4:22); da ol tohtavčular anyn qatyna ševeti Jissaharnyn da n. ulanlaryna Jissaharnyn Netan’el uvlu Cu'arnyn (Num 2:5); da n. ulanlaryna Zevulunnun Eliav uvlu Helonnun (Num 2:7); da n. ulanlaryna Ru'uvennin Elicur uvlu Šede'urnun (Num 2:10); da n. ulanlaryna Šim 'onnun Šelumi'el uvlu Curišadajnyn (Num 2:12); passim 
-d́an: da sözĺadi Moše ulanlaryna Jisra’elnin da berdilar anar bar nasilari Jisra’elnin biŕar tajaq n. biŕar tajaq n. üvüsajyn (Num 17:21)

-ler: da ündedi alarny Moše da qajttylar anar Aharon da bar ol n. క̌ymatta da sözledi alarga Moše (Exo 34:31); da ol n. keltirdiler ošol ol šoham tašlaryn efodg்a da ošol ol milu’im tašlaryn ḩošenge (Exo 35:27) $\Rightarrow$-lar

-leri: da keldiler bar n. ol žymatnyn da anlattylar Mošege (Exo 16:22) $\Rightarrow$-ĺari

-lar: alġyšlarmen any da jajarmen any da arttyryrymen any astry astry on eki $n$. tuvdurur da qojarmen any ullu hạlyqqa (Gen 17:20); alardylar n. ol ševetlarnin alar edilar ol turuvčular ol sanalganlar qatyna (Num 7:2); da juvuttular ol $n$. ošol qutlamaġyn ol mizbeahnyn (Num 7:10); da juvuttular ol n. ošol qarbanlaryn özlarinin (Num 7:10); da eger birbyla tartsalar da jyštyrylsynlar saja bar ol n. agjalyqlary minlarinin Jisra’elnin (Num 10:4); passim $\Rightarrow$-ler

-ĺard́an: on eki syg்yr araba eki ol $n$. da ögüz bird́an da juvuttular alarny alnyna ol miškannyn (Num 7:3)

-ĺari: bulardylar ünd́almüšlari ol క̌ymatnyn n. ševetlarinin atalarynyn (Num 1:16); bulardylar ol sanalġanlar ki sanady Moše da Aharon da n. Jisra’elnin on eki kiši biŕar kiši üvüsajyn atalarynyn edilar (Num 1:44); da sanady Moše da Aharon da n. ol žymatnyn (Num 4:34); bar ol sanalganlar ki sanady Moše da Aharon da n. Jisra'elnin ošol ol Levililarni (Num 4:46); da juvuttular ol $n$. Jisra'elnin aġaraqlary üvünün atalarynyn (Num 7:2); passim $\Rightarrow$-leri

-ĺarind́an: budur qutlamaġy ol mizbeahnnyn jaġyndyrylgan künd́a any n. Jisra’elnin (Num 7:84); sözlagin ulanlaryna Jisra’elnin da algynn alardan birar tajaq üvüsajyn atanyn bar n. alarnyn (Num 17:17)

-larinin: da nasisi n. ol Levinin El'azar uvlu Aharonnun ol kohennin (Num 3:32)

-ĺarińa: da ajttylar Mošeǵa da El'azarġa ol kohenǵa da n. ol žymatnyn (Num 32:2)

-ĺarnin: alnynda Mošenin da alnynda El'azarnyn ol kohennin da alnynda ol n. bar ol 3̌ymatnyn (Num 27:2); da sözla dilar alnynda Mošenin da alnynda ol n. ag்araqlarynyn atalarnyn ulanlarynyn Jisra'elnin (Num 36:1)

-ni: törečini qarġamag்yn da n. ulusujda sökmegin (Exo 22:27); ki men beŕamen any ulanlaryna Jisrảelnin birar kiši birar kiši ševetindán atalarynyn ijijiz bar $n$. alarda (Num 13:2)

-si: da n. üvünün atanyn uruvunun Geršoninin Elijasaf uvlu La'elnin (Num 3:24); da n. üvünün atanyn uruvlarynyn ol Qahatinin Elicafan uvlu 'Uzielnin (Num 3:30); da n. nasilarinin ol Levinin El'azar uvlu Aharonnun (Num 3:32); da n. üvünün atanyn uruvlarynyn Merarinin Curiel uvlu Avihajilnin (Num 3:35); ol ekinči künd́a juvuttu Netan'el uvlu Cu'arnyn n. Jissaharnyn (Num 7:18); passim

-sinin: išibyla Kazbinin qyzynyn n. Midjannyn (Num 25:18)

\& Heb. נָשִׁיא 'prince', Mod.SWKar. nasi 'prince'.

navi'a 'prophetess'

aldy Mirjam ol n. tuvduġu Aharonnun ošol ol tafny qoluna (Exo 15:20)

e Heb. נְבָיאָה 'prophetess'. 
nazir 'nazirite; consecrated person'

kiši \{jemeśa qatyn\} ki ajyrsa nijet etŕa nijet bolma $n$. (Num 6:2); jülütsün ol $n$. ešigind́a ohel moédnin (Num 6:18); da andan sortun ičsin ol n. vina (Num 6:20)

-nin: budur üvŕatüvü ol n. (Num 6:13); da bersin uvučlary üstüńa ol n. jülütḱnind́an sortun ošol čačyn nazirliginin (Num 6:19); budur üvratüvü n. (Num 6:21)

\& Heb. נָזזְר '1. of high rank; 2. consecrated', Mod.SWKar. nazir '1. dedicated to God; 2. prince'.

nazirli|k 'being na nazarite; naziritehood'

n. künlarind́a baryndan neki qylyndy borlalyqtan (Num 6:4); n. künlarind́a azizdir ol Adonajga (Num 6:8); jülütsün ol nazir ešiginda ohel mo'ednin ošol n. čačyn bašynyn da alsyn \{ošol\} n. čačyn bašynyn da bersin ol ot üstüna ki ol šelamim debehasy tübüńa (Num 6:18)

-|gi: murdar boldu n. anyn (Num 6:12); budur üvŕatüvü nazirnin ki nijet etśa qarbany anyn Adonajġa n. üčün bašqa neki jetśa küčü qolunun nijetińa köŕa ki nijet etśa alaj qylsyn üvŕatüvüna köŕa nazirliginin (Num 6:21)

-|ginin: nijetli künlarind́a n. (Num 6:5); murdar etśa bašyn n. (Num 6:9); budur üvŕatüvü ol nazirnin tolġan tolġan künd́a künlari n. keltirsin özün ešigina ohel mo'ednin (Num 6:13); budur üvratüvü nazirnin ki nijet etśa qarbany anyn Adonajġa nazirligi üčün bašqa neki jetśa küčü qolunun nijetińa köra ki nijet etśa alaj qylsyn üvratüvüńa körán. (Num 6:21)

e Heb. נָזִיר '1. of high rank; 2. consecrated' + Kar. deriv. suff., Mod.SWKar. nazir '1. dedicated to God; 2. prince'.

nendij '1. what, what kid of; 2. whichever, any'

da ajtty Avrame Adonaj Tenri n.jal berirsen maja (Gen 15:2); biz bilmejbiz n. ̌̌ynsy byla debehanyn qulluq eterbiz Adonajga (Exo 10:26); haligine bildim ki ulluraqty Adonaj bar ol malahlardan ki n. iš byla ki čajalyq ettiler alaj qajtardy tölev alarġa (Exo 18:11); ki berse kiši dostuna ešek jemese ögüzjemese qoj da n. d́a tuvar saqlama (Exo 22:9); da haligine barg்yn köndergin ošol ol ulusnu ol orunġa ki sözledim saja muna malahym baryr alnyjda da sag்yngan künümde n. jazyqlaryn alarnyn da karatetermen alarny buda jazyġy üčün alarnyn (Exo 32:34); passim

e Mod.NWKar. nińdi nińdij ‘1. what, what kid of; 2. any', Mod.SWKar. nendi nendij id.

nedir: nedir saja 'what is wrong to you?, what happened?'

da ajtty anar n. s. e Hagar (Gen 21:18)

e Mod.NWKar. ne 'what', Mod.NWKar. saja 'you (dat.)'.

neqeva 'female'

-nyn: türsünün erḱkaknin jemeśa n. (Deu 4:16)

^ Heb. נְקָבָּ 'female'.

nes 'sign'

-ḱa: örtaǵand́a ol ot ol eki jüz da enli kišini da boldular n. (Num 26:10)

\& Heb. נي 'sign'. 


\section{neseh 'drink offering'}

da $q[u]$ jdu anyn üstüna $n$. da $q[u]$ jdu anyn üstüńa jav (Gen 35:14); onunču ülüšü ol efanyn özek jumurulgan jančqan jav byla dörtünčü ülüšü ol hinnin da n. dörtünčü ülüšü ol hinnin (Exo 29:40); tirki čygarmajyz anyn üstüne da n. q[u]jmajyz anyn üstüne (Exo 30:8); bir qozuġa qodešta qujma n. esirtüvčü alnynda Adonajnyn (Num 28:7)

-i: qozunu qylg̀yn ol eki ingirler arasyna tirkisikibik ol ertenbylanyn da nesehi kibik anyn qylġyn anar (Exo 29:41); da n. anyn čag்yr dörtünčü ülüšü ol hinnin (Lev 23:12); da tirkisi anyn da n. kečinḿakḱ köŕa (Num 15:24); n. anyn dörtünčü ülüšü ol hinnin (Num 28:7); da eki 'esronlar öźak tirki jumurulgan javbyla da n. anyn (Num 28:9); har šabatnyn šabatynda olasy qatyna ol hammešalik qarbannyn da n. anyn (Num 28:10); passim

-in: qylsyn ol kohen ošol tirkisin da ošol n. anyn (Num 6:17)

-ind́an: bašqa ol hammeśalik 'oladan tirkisind́an da n. anyn (Num 29:16)

-ḱa: čag்yr n. dörtünčü ülüšü ol hinnin (Num 15:5); čag்yr n. üčünčü ülüšü ol hinnin (Num 15:7); čaġyr juvutqun n. jarymy ol hinnin (Num 15:10)

-lar: juvutma otlu qarban Adonajga 'ola da tirki debeha da n. (Lev 23:37)

-ĺari: bolsunlar ola Adonajġa da tirkilari alarnyn da n. alarnyn (Lev 23:18); četan macalar öźak halalar jumurulganlar javbyla da juġa macalar jag்ylganlar javbyla tirkilar alarny da n. alarnyn (Num 6:15); n. alarnyn jarymy ol hinnin bolsun ol bir tanag்a (Num 28:14); bularny qylyjyz tüǵallar bolsunlar sizǵa da n. d́a alarnyn (Num 28:31); n. alarnyn kečinḿaklarińa köŕa (Num 29:6); passim

-ĺarijiz: bašqa nijetlarijizd́an da žomartlyqlaryjyzdan 'olalaryjyzdan da tirkilarijizsajyn da n. sajyn da šelamimlarijizsajyn (Num 29:39)

-ĺarind́an: bašqa hatatyndan ol kipurimnin da ol hammešalik oladan da tirkisindan da n. alarnyn (Num 29:11)

\& Heb. נקסֶָ 'drink offering'.

neteq 'scab'

baqsyn ol kohen ošol ol hastalyqny ol jedinči künd́a da muna jajylmady ol n. (Lev 13:32); da muna jajylmady ol n. terid́a (Lev 13:34); eger jajylmajajylsa ol n. terid́a arynganyndan sortun (Lev 13:35); baqsyn any ol kohen da muna jajyldy ol n. terida (Lev 13:36); eger türsününd́a tursa ol n. da qara čač ösśa anda ong்aldy ol n. aruvdu ol da aruv etsin any ol kohen (Lev 13:37)

-ka: budur ol üvŕatüv bar hastalyg்yna ol cara'atnyn da n. (Lev 14:54)

-ni: jülünsün da ošol ol n. jülümasin da beklasin \{[o]lkohen $\}$ ošol ol n.jedi künlar (Lev 13:33); baqsyn ol kohen ošol ol n. ol jedinči künd́a (Lev 13:34)

-nin: ki körśa ol kohen ošol hastalyg்yn ol n. da muna tüvüldü körümü anyn teŕanŕak ol teridá da qara čačjohtu anda da beklasin ol kohen ošol hastalyg்yn ol n. jedi künlar (Lev 13:31); körümü ol n. teŕanŕak tüvüldü ol teridan (Lev 13:32)

-ti: murdar etsin any ol kohen n. ol cara'at ol bašnyn jemeśa ol sajalnyn ol (Lev 13:30)

e Heb. נֶתק 'scab'. 
ni'ufluq et- 'to commit adultery'

-megin: $n$. e. (Exo 20:14) $\Rightarrow$-magin

-magin: $n$.e. (Deu 5:18) $\Rightarrow$-megin

-śa: kiši ki n[i]'ufluq e. qatynybyla kišinin ki n. e. qatynybyla dostunun ölḿa ötürülsün (Lev 20:10)

-üvčü: ölḿa ötürülsün ol n. e. kiši da ol n. e. qatyn (Lev 20:10)

e Heb. נִאוּ 'adultery, prostitution' + Kar. deriv. suff. + Kar. aux. verb.

O, ' O

odv́erja '1. lintel; 2. pl. side posts; 3. upper door post'

da körer ošol ol qanny ol üst o. üstüne da eki ol odv́erjalar üstüne (Exo 12:23)

-ga: alyjyz bav čabor da mančyjyz qanġa ki savutta da tijijiz ol üst o. da eki ol odv́erjalarga ol qandan (Exo 12:22); da juvutsun any ol töreči ol ešikke jemese ol o. da čančsyn biji anyn ošol qulaġyn anyn (Exo 21:6)

-lar: alsynlar ol qandan da bersinler ol eki o. üstüne da ol üst odv́erja üstüne ol üvler üstüne ki ašasalar any alarda (Exo 12:7); da körer ošol ol qanny ol üst odv́erja üstüne da eki ol o. üstüne (Exo 12:23)

-larğa: alyjyz bav čabor da mančyjyz qang்a ki savutta da tijïiz ol üst odv́erja da eki ol o. ol qandan (Exo 12:22)

-larny: qyjasa jazyp tutqun alarny o. üstüna üvlarijiznin (Deu 6:9)

-lary: jazġyn alarny o. üstüne üvlerijnin (Deu 11:20)

e Russ. arch. одверье (sg.), одверья 'door-frame’, Mod.SWKar. odv́erja 'door'.

ohel mo'ed 'ten of meeting'

o. m. tyšqartyn parohetke (Exo 27:21); bergin any 0 . $m$. byla mizbeah arasyna (Exo 3०:18); da atady anar o. m. (Exo 33:7); tüǵallaśa bošatlyq qolmaqtan ol qodeš üčün da o. m. üčün (Lev 16:20); bošatlyq qolsun ol aziz miqdaš üčün da o. m. üčün (Lev 16:33); passim

-de: berdi ošol ol stolnu o. m. (Exo 40:22); qojdu ošol ol čyraqbany o. m. (Exo 40:24); qojdu ošol altyn mizbeaḥny o. m. (Exo 40:26); turujuz o. m. (Deu 31:14); da bardy Moše da Johošua'da turdular o. m. (Deu 31:14); passim $\Rightarrow$-d́a

-den: ündedi Mošeni da sözledi anar šehinasy Adonajnyn o. m. ajtadoġač (Lev $1: 1)$

-d́a: tütüsünün ol otjamlarnyn alnynda Adonajnyn ki o. m. (Lev 4:7); bersin müvüzlari üstüńa ol mizbeaḥnyn ki alnynda Adonajnyn ki o. m. (Lev 4:18); bird́a adam bolmasyn o. m. (Lev 16:17); tyšqartyn parohetińa ol šarajatnyn o. m. qyjaslasyn any Aharon (Lev 24:3); sözladi Adonaj Mošeǵa midbarynda Sinajnyn o. m. (Num 1:1); passim $\Rightarrow$-de

-ge: kelgenlerinde o. m. (Exo 28:43); ornuna qajsy kelir o. m. jumuš etḿa qodešte (Exo 29:30); kelgenlerind́a o. m. juvsunlar (Exo 30:2); edi bar izlevčü sorma sözün 
Adonajnyn čýgaredi o. m. ki tyšqartyn avulġa (Exo 33:7); bar savutlaryn qullug்unun ol miškannyn o. m. (Exo 39:40); passim $\Rightarrow$-ǵa

-ǵa: keltirsin any o. m. (Lev 4:5); kijirsin ol jag்yndyrylgan kohen qanyndan ol tananyn o. m. (Lev 4:16); kijirilśa qandan anyn o. m. (Lev 6:23); keldi Moše da Aharon o. m. (Lev 9:23); kelgenijiź́a o. m. (Lev 10:9); passim $\Rightarrow$-ge

-ni: aziz etermen ošol o. m. (Exo 29:44); jag்yndyrg்yn anyn byla ošol o. m. (Exo 30:26); ošol o. m. da ošol ol aronnu (Exo 31:7); eltsinlar ošol enlarin ol miškannyn da ošol o. m. (Num 4:25)

-nin: Aharonnu da ošol avullaryn anyn juvutqun ešigine o. m. dajuvg்un alarny suvbyla (Exo 29:4); juvutqun ošol ol tanany alnyna o. m. (Exo 29:10); sojg̉un ošol ol tanany alnynda Adonajnyn ešigind́a o. m. (Exo 29:11); ošol ol ötmekni ki četende ešiginde o. $m$. (Exo 29:32); hammešelik 'ola dorlajyzsajyn eši $[$ g]inde o. m. (Exo 29:4); passim

\& Heb. אה:ל מוֹעֵ 'tent of meeting; tabernacle of meeting'.

ojuv 'filigree (setting for precious stones)'

-lar: tǔ̌urg்un ošol eki ol tašlarny atlarybyla uvullarynyn Jisra’elnin quršalġanlar altyn o. byla qylg̀yn alarny (Exo 28:11); qylg̀yn altyn o. (Exo 28:13); bergin ošol synžyrlary ol örkenler išinin ol o. üstüne (Exo 28:14); qyldylar ošol ol šoham tašlaryn quršalganlar altyn o. byla (Exo 39:6); ol dörtünčü jerge taršiš šoham da jašfe quršalġanlar altyn o. byla tolturmaqlarynda (Exo 39:13); qyldylar eki altyn o. da eki jüzükler altyndan (Exo 39:16); ošol eki učlaryn eki ol örkenlernin berdiler eki ol o. üstüne (Exo 39:18)

e Mod.NWKar. ojuv 'pattern; embroidery'. $\Rightarrow$ ojuvla-, ojuvlan-

ojuvla- 'to set (precious stone in a setting)'

-ma: o. ojuvlar išleme altyn byla da kümüš byla da tuč byla (Exo 31:4); o. ojuvlar qylma altyn byla da kümüš byla da tučbyla (Exo 35:32)

e Mod.NWKar. ojuv 'pattern; embroidery' $\Rightarrow$ ojuv, ojuvlan-

ojuvlan- 'to be set (precious stone in a setting)'

-ganlar: dörtünčü jerge taršiš tašy da šoham da jašfe o. altyn byla bolsunlar tolturmaqlarynda (Exo 28:20)

\& Mod.NWKar. ojuv 'pattern; embroidery'. $\Rightarrow$ ojuv, ojuvla-

ol 'the'

arasyna o. jaryqnyn da arasyna o. qaran[g்ylyqnyn] (Gen 1:18); qozlasynlar o. suvlar qozlamaġyn (Gen 1:20); qu[š učqa]lasyn o.jer üstüńajuvuq raqaiasyna o. kökler[nin] (Gen 1:20); bar tiri žanny o. tebrenedoġanny [ki qoz]ladylar o. suvlar žynslary sajyn (Gen 1:21); da o. quš [art] syn jerd́a (Gen 1:22); passim

e Mod.NWKar. ol '1. that; 2 . he, she it'.

olturuš ' 'place of dwelling'

da bar ulanlaryna Jisra’elnin edi jaryq o. orunlarynda (Exo 10:23); kiši ki satsa o. üv qalaly šaharda (Lev 25:29)

-lary: o. alarnyn bašlap Mešadan kelüvüj sary Sefaraġa tavy sary ol mizrahnynn (Gen 10:30) 
-laryjyzda: heč qučurajġanny ašamajyz bar o. ašajyz macalar (Exo 12:20); jandyrmajyz ot bar o. ol šabat kününde (Exo 35:3); bar o. heč javny da heč qanny ašamajyz (Lev 3:17); heč qanyy ašamajyz bar o. qušnun da tuvarnyn (Lev 7:26); heč iš qylmajyz šabatty ol Adonajg̉a bar o. (Lev 23:3); passim

-laryjyzdan: o. keltirijiz ötmiagin tenufanyn (Lev 23:17)

-laryjyznyn: kelśajiz jerińa o. (Num 15:1)

-larynda: bar šaharlaryn alarnyn o. da ošol bar sarajlaryn alarnyn küvdürdülar otta (Num 31:10)

-u: jalġyz oltursun tyšqartyn avulġa bolsun o. anyn (Lev 13:46)

-uj: muna semiz orunlarynda ol jernin bolur o. senin (Gen 27:39); da ajtty kim orundady o. (Num 24:21)

-una: da aldy ... ošol Hag்arny ol Micrilini qaravašyn özünün aḥerind́an on jyllarnyn o. Avramnyn jerinde Kena'annyn (Gen 16:3); tašqyny ol öźanlarnin ki qurdu o. Arnin da tajandy čegińa Mo’avnyn (Num 21:15)

e Mod.NWKar. olturuš 'seat'. $\Rightarrow$ olturušs ${ }^{I}$

olturušII 'inhabited'

ulanlary Jisra’elnin ašadylar ošol ol manny qyrq jyl kelgenlerinedejin o. jerge (Exo 16:35)

e Mod.NWKar. olturuš 'seat'. $\Rightarrow$ olturuš ${ }^{\mathrm{I}}$

'omer 'omer (a dry measure), a tenth part of an ephah'

budur ol söz ki bujurdu Adonaj čöplejiz andan kiši jemini köre ó ündeledogan ölčüv bašsajyn (Exo 16:16); ölčedilar 'o. ündeledogan ölčüv (Exo 16:18); ol ó. ündeledoġan ölčürnü man (Exo 16:32); ajtty Moše Aharonġa alg̀yn bir qošun da bergin ary tolu 'o. ündeledog்an ölčüvnü man (Exo 16:33); ol 'o. onunču ülüšü ol efanyn edi ol (Exo 16:36)

\& Heb. עמקר 'omer (a dry measure), a tenth part of an ephah'.

on ökövlen '(coll.) twelve'

o. ö. qullaryj qaryndašlardyr biz uvullary bir kišinin (Gen 42:13)

e MWKar. ekövlen '(coll.) two'; due to assimilation. $\Rightarrow$ ököv, ökövlen, önökövlen oq: oq atuvču 'archer'

da edi bolušluġu Tenrinin ol ulanbyla da ulġajdy da olturdu midbarda da edi o. a. da jajačy (Gen 21:20)

¿ Mod.SWKar. ok atuvcu 'archer'.

oruš- 'to wage war'

-ma: nečik bujurdu Adonaj ki barġajlarjasanġanlarčerüvge alnynda qaryndašlarynyn o. ašyp ol Jardenni olturuvčularybyla jerinin Kena'annyn (Deu 33:21)

e Mod.NWKar. uruš- 'to wage war, to figth', Mod.SWKar. orus- id.

osallan- 'to be lazy, to be idle'

-asiz: ajtty o. siz o. anyn üčün siz ajtasiz barajyq debeḥa etejik Adonajgia (Exo 5: 17) 
-dylar: ošol qyjasyn ol kirpičlernin ki alar qyladylar tünelej biri künlej qojujuz alar üstüne eksitmejiz andan ki o. alar (Exo 5:8)

e Mod.NWKar. osallan- 'to hesitate'.

osnova 'warp'

ol upraq jemeśa ol o. jemeśa ol utok (Lev 13:58)

-da: jemeśa o. jemeśa utokta üskülünün (Lev 13:48); eger jajyldy eśa ol hastalyq upraqta jemeśa o. jemeśa utokta (Lev 13:51); muna jajylmady ol hastalyq upraqta jemeśa o. jemeśa utokta (Lev 13:53); eger körünśa dag்yn upraqta [je]meśa o. jemeśa utokta (Lev 13:57)

-dan: jyrtsyn any ol upraqtan jemeśa ol teridan jemeśa ol o. jemeśa ol utoktan (Lev $13: 56)$

-ny: küvdürsün ošol ol upraqny jemeśa ol o. jemeśa ošol ol utoknu junda jemeśa üskülüda (Lev 13:52)

-nyn: budur üvŕatüvü hastalyġynyn ol cara'atnyn ol upraqnyn jundan jemeśa üskülüd́an jemeśa o. jemeśa ol utoknun (Lev 13:59)

e Russ. основа id., cf. Pol. оsnowa 'warp', Ukr. основа id. In K, it is likely of Russian origin in the light of the (Russian) $\Rightarrow$ utok 'woof' used in the translation.

otalav 'poison'

-ǵa: janġaj künlarǵadejin neginća ki čyqqaj burnujuzdan da bolur sizǵa o. (Num 11:20)

e Mod.SWKar. otalav 'poison'.

ovellikka *'permanently, forever'

ol jer satylmasyn o. ki menimdi ol jer ki ġariplar da očarlardyr siz birǵaḿa (Lev 25:23); eger julunmasa tolġunča anar tüǵal jyl da qajjam bolur ol üv ki šaharda ki anar qala o. satyn aluvčuġa any dorlarysajyn čyqmasyn joveld́ad́a (Lev 25:30)

^ Perhaps related to jovel 'jubilee' + the Kar. -lik deriv. suff., cf. Heb. יוֹיבל 'jubilee, year of jubilee'; the word-initial $j$ - could have been reinterpreted as a prothetic sound. Cf.

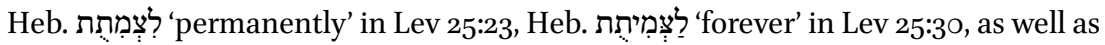
EKar. bašbatra 'forever' used in C (Lev 25:23, Lev 25:30). The equivalents of this word in other translations are ovellikke (in $\mathrm{H}$ ), baš batra (in M), ömürlükka (in F305-O1). $\Rightarrow$ jovel, jovel: jovelge dejin

Ö

önökövlen '(coll.) twelve'

-dir: ö. biz qaryndašlar uvullary atamyznyn (Gen 42:32)

e MWKar. on ekövlen '(coll.) two'; due to assimilation. $\Rightarrow$ on ökövlen, ököv, ökövlen

ököv '(coll.) two'

da elttilar üśak byla ö. d́a (Num 13:23)

e MWKar. eköv '(coll.) two'; due to assimilation. $\Rightarrow$ on ökövlen, ökövlen, onökövlen 
ökövlen '(coll.) two'

quvadyr bir kiši minni da ö. qačyradylar tümenni (Due 32:30)

e MWKar. ekövlen '(coll.) two'; due to assimilation. $\Rightarrow$ on ökövlen, ököv, onökövlen ökün- *'to get angry'

-dülar: da ö. anyn üstüńa (Gen 4:23)

ölčöv 'measure, weight'

bir ö. bar ol enlerge (Exo 26:2); bir ö. onbirside enlerge (Exo 26:8); bir ö. bar ol enlerge (Exo 36:8); bir ö. on bir ol enlerge (Exo 36:15); da qajtaryrlar ötḿagijizni ö. byla da ašarsiz da tojmassiz (Lev 26:26)

-ü: da aldy ol kiši altyn syrg்a gög ö. anyn (Gen 24:22); da muna kümüšü kišinin avzunda qabynyn kümüšümüz ö. byla (Gen 43:21)

-lari: da eki beleziklar qojdu qollary üstüńa anyn on altyn ö. alarnyn (Gen 24:22)

^ Mod.NWKar. ölč́av 'measure'. $\Rightarrow$ ölčüv

ölčüv 'measure, weight'

'omer ündeledog்an ö. (Exo 16:16); ölčedilar 'omer ündeledog்an ö. byla (Exo 16:18); čöplediler ötmek eki anča eki ol ö. (Exo 16:22)

-d́a: kümüš ölčüvünd́a da jüvüš ö. (Lev 19:35)

-nü: 'omer ündeledog்an ö. (Exo 16:32); 'omer ündeledogan ö. (Exo 16:33)

-ü: otuz mitqal ö. anyn (Num 7:13); jetmiš mitqal ö. anyn mitqaly byla ol qodešnin (Num 7:13); otuz mitqal ö. anyn bir kubok kümüš ö. anyn jetmiš mitqal mitqaly byla ol qodešnin (Num 7:19); otuz mitqal ö. anyn (Num 7:25); passim

-ünd́a: kümüš ö. da jüvüš ölčüvd́a (Lev 19:35)

e Mod.NWKar. ölćáv 'measure'. $\Rightarrow$ ölčöv

örten- 'to be consumed by fire, to be burned down'

-mejdi: ol tegenek janady otta da ol tegenek ö. (Exo 3:2)

-se: čyqsa ot da örtese tegeneklerni da ö. styrta jemese ol turuq (Exo 22:5)

\& Mod.NWKar. örtal- 'to be burned down'.

ötmek: eki jüzlü ötmek 'showbread'

bergin ol stol üstüne e.j. o. (Exo 25:30)

-ni: da ošol ol e.j. ö. (Exo 35:13); biširirediler ošol e.j. ö. (Exo 37:16); da ošol e.j. ö. (Exo 39:36)

-nin: e.j. ö. (Exo 40:23)

e Mod.NWKar. ötmáak 'bread', Mod.NWKar. eki juźlu 'of two faces'. $\Rightarrow$ ötmak

ötḿak: eki jüzlü ötḿak 'showbread'

-nin: stolu üstüńa ol e.j. ö. jajsynlar kök upraq (Num 4:7)

e Mod.NWKar. ötmak 'bread', Mod.NWKar. eki juźlu 'of two faces'. $\Rightarrow$ ötmek

öz- 'to crush'

-ersen: bolġaj ki nečik küčejśaj da ö. bojunsasyn anyn bojnujüstünd́a (Gen 27:40)

e Mod.NWKar. eź- (!) 'to crush', Mod.SWKar. ez- 'to crush'.

öze|k 'fine flour'

onunču ülüšü ol efanyn ö.jumurulgan jančqan jav byla (Exo 29:40) 
-|ginden: ötmek macalar da halalar macalar jumurulġanlar javbyla da juġa macalar jag்lġanlar javbyla ö. budajlarnyn qylgyn alarny (Exo 29:2)

e Mod.NWKar. öźak un 'fine flour', cf. Mod.NWKar. öźak 'fine, excellent'. $\Rightarrow$ öźak özele $\mid \mathbf{k} *$ 'desire’

-|gij: da erija ö. da ol erklenir send́a (Gen 3:16)

e Mod.EKar. özle- 'to want, to strive for'.

öźa|k 'fine flour'

క̌an ki juvutsa qarban tirki Adonajġa ö. bolsun qarbany anyn (Lev 2:1); ki juvutsaj qarban tirki biširilǵan pečta ö. halalar macalar jumurulganlar javbyla (Lev 2:4); eger tirki išlanǵan ol tava üstüńa bolsa qarbanyj ö. jumurulgan javbyla maca bolsun (Lev 2:5); eger tirki išlanǵan panv́ada bolsa qarbanyj ö. javbyla qylynsyn (Lev 2:7); onunču ülüšün ol efanyn ö. hatatqa qojmíasin anyn üstüńa jav (Lev 5:11); passim

-|gind́an: keltirsin any uvullaryna Aharonnun ol kohenlarǵa da ajyrsyn andan tolu uvuču ö. da javyndan anyn (Lev 2:2); ajyrsyn andan qoš uvuču byla ö. ol ti [rki]nin da javyndan anyn (Lev 6:8)

e Mod.NWKar. öźak un 'fine flour', cf. Mod.NWKar. öźak 'fine, excellent'. $\Rightarrow$ özek

$\mathbf{P}, \mathbf{P}$

panv́a '(frying) pan'

-da: eger tirki išlanǵan p. bolsa qarbanyj öźak javbyla qylynsyn (Lev 2:7); bar tirki ki biširilśa pečta da bar qylynġan p. da tavaüstüna kohenǵa ol juvutuvčuġa anar bolsun (Lev 7:9)

\& MPol. panwia 'a pan, a shallow cauldron', see Linde (1811: 623, s.v. panew), SStp. (IV: 22, s.v. panew).

parohet 'curtain, veil'

qylg̀yn p. kökten da jipkinden da qyrmyzy jipekten da ešken bezden išin ojuvlavčunun qylsyn any keruvim (Exo 26:31); ajyrsyn sizge ol p. arasyna ol qodešnin da arasyna qodeš ol qodešnin (Exo 26:33)

-in: ošol p. ol qaplavny (Exo 39:34); da qojdu ošol p. ol qaplavnyn (Exo 40:21); kelsin Aharon da uvullary anyn köčkand́a ol avul da endirsinlar ošol p. ol qaplavnyn (Num 4:5)

-inin: bürksün ol qandan jedi keŕatlar alnynda Adonajnyn alnynda p. ol qodešnin (Lev 4:6); bürksün jedi keŕatĺar alnynda Adonajnyn alnynda p. ol qodešnin (Lev 4:17) -ińa: tyšqartyn p. ol šarajatnyn ohel moedd́a qyjaslasyn any Aharon (Lev 24:3) -ke: kijirgin ary ičkertin p. ošol ol šarajtny (Exo 26:33); qojg்un ošol ol stolnu tyšqartyn p. (Exo 26:35); ohel mo'ed[de] tyšqartyn p. ki ol šarajat üstüne (Exo 27:21); berdi ošol ol stolnu ohel mo`edde jany üstüne ol miškannyn cafon sary tyšqartyn p. (Exo 40:22) $\Rightarrow$-ka -ka: ajtty Adonaj Mošeǵa sözlagin Aharonġa qaryndašyja da kelmasin har vahtta ol qodeška ičkartin p. alnyna ol kaporetnin (Lev 16:2); kijirsin ičkartin p. (Lev 16:12); 
keltirsin ošol qanyn anyn ičkartin p. (Lev 16:15); tek ol p. kelḿasin (Lev 21:23); saqlajyz ošol kohenligijizni bar išisajyn ol mizbeahnnyn da ičkartin p. (Num 18:7) $\Rightarrow$-ke -ni: bergin ošol ol p. ol eslar tübüne (Exo 26:33); ošol ol kaporetni da ošol p. ol qaplavny (Exo 35:12); qyldy ošol p. kökten da jipkinden da qyrmyzyjipekten (Exo 36:35); qojgiun anda ol aronun ol šarajatnyn da qaplag̉yn ol aron üstüne ošol ol p. (Exo 40:3)

-nin: alnynda ol p. (Exo 30:6); ošol tabanlaryn ol p. jüz tabanlar jüz ol qantardan qantar tabanġa (Exo 38:27); qojdu ošol altyn mizbeahnny ohel mo'edde alnynda ol p. (Exo 40:26)

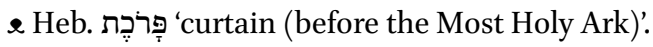

ṕeč: kireč ṕeči kiŕač ṕeči 'lime kiln'

-nin: muna kötürüldü qoju tütünü ol jernin tütünü kibik ol kiŕač ṕ. (Gen 19:28); alyjyz özüjüzǵa tolu qoš uvučlaryjyzny sof̣̣asyn kireč ṕ. da bürksün any Moše ol köklerge (Exo 9:8); aldylar ošol sophasyn ol kireč ṕ. da turdular alnynda par'onun da bürktü any Moše ol köklerǵa (Exo 9:10); kötürüldü tütünü kibik ol kireč ṕ. da qaltrady bar ol tav astry (Exo 19:18)

e Russ. печь 'furnace, stove', ModNWKar. ṕeč id., ModNWKar. kirać 'lime'.

peḥetet 'sunken spot (in leprosy)'

otta küvdürgün any p. ünd́aĺadog்an hastalyqty ol artynda jemeśa alnynda (Lev 13:

55)

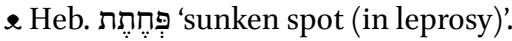

perepelice 'quail'

da edi ingirde da kötürüldü ol p. (Exo 16:13)

^ Ukr. nерепелиия ‘quail', cf. Russ. dial. перепелица id., see Černych (II: 21, s.v. nepe$n e \Omega) . \Rightarrow$ perepelića

perepelića 'quail'

alġyn maja üč bozov da üč ečki da üč qočqar da bedeńa da p. (Gen 15:9)

-ni: da turdu bar ol ulus bar ol künnü da bar ol kećani da bar ol tanbylag்yda künnü da jyštyrdylar ošol ol $p$. (Num 11:32)

e Ukr. перепелищя 'quail'; cf. Russ. dial. перепелища id. (Černych II 21, s.v. nерепел) $\Rightarrow$ perepelice

peres 'bearded vulture'

-ni: da ošol bunu jiŕanči etijiz ol quštan ašalmasynlar qusqunčudur alar ošol ol nešerni da ošol ol p. da ošol ol 'aznijany (Lev 11:13); budur ki ašamajyz alardan ol nešerni da ol p. da ol 'aznijany (Deu 14:12)

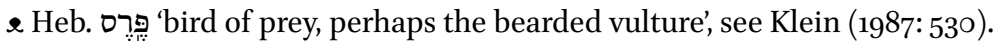

pižma *'trinket'

keltirdiler halḥa da syrg்a da jüzük da p. bar altyn savut da bar kiši ki sundu tanufasyn altynnyn Adonajnyn (Exo 35:22); altyn savut ajaq bilazigi da qol bilazigi jüzük halha da p. bošatlyq qolma క̌anlarymyz üčün alnynda Adonajnyn (Num 31:50)

e Mod.SWKar. pizma 'trinket', perhaps related to Pol. piżmo 'musk'. 
pole 'field'

soġagny da jürnü da bojvolnu da p. qojun da dišonnu (Deu 14:5)

\& Pol. pole 'field', Russ. nоле id., Ukr. поле id. $\Rightarrow$ polnyj

polnyj adj. 'field'

alsynlar cara'attan arynuvču kišiǵa eki p. qušlar (Lev 14:4)

\& Pol. polny '(adj.) field' with ESlav. ending. $\Rightarrow$ pole

priṕečka 'stove'

ṕeč da p. qavšatylsyn murdarlardylar alar (Lev 11:35)

\& Russ. dial. припечка '1. stove; 2. stove bench', see SRNG (XXXI: 432).

pusta et- 'to desolate, to bring to desolation'

-'armen: berirmen ošol šaharlaryjyzny veŕan da p. e. ošol miqdašlaryjyzny da qabul etḿanmen ij qabullug்ujuznu (Lev 26:31)

e Pol. pusty 'empty', Mod.NWKar. pusta 'empty' + Kar. aux. verb.

Q

qa'at 'pelican'

-ny: ošol ol tinšemetni da ošol ol q. da ošol ol raḥamny (Lev 11:18); ol qa’a[t]ny da ošol raḥamany da ošol ol šalahny (Deu 14:17)

e Heb. קָָָָ 'pelican', see Klein (1987: 559), 'a species of owl (?)', see Koehler \& Baumgartner (1985: 817).

qabulluq '1. acceptance; 2 . pleasure'

-qa: juvutsun any q. özü üčün alnyna Adonajnyn (Lev 1:3); debeḥa etśajiz šelamim debehasy alnynda Adonajnyn q. özüjüz üčün (Lev 19:5); q. özüjüzüčün tüǵal erkakni (Lev 22:19); bolmastyq. siznin üčün(Lev 22:20); tüǵal bolsun q. hečajip bolmasyn anda (Lev 22:21); passim

\& Mod.SWKar. qabulluk 'pleasure', Mod.NWKar. qabuyluḥ 'acceptance'. $\Rightarrow$ ij qabulluq qacat *'a small part'

-y: ajtty Moše qajnatasyna ki keledi maja q. ol ulusnun sorma (Exo 18:15)

\& Heb. קָָָָ '1. a little; 2. part, a small part'.

qačan: qačan ki 'since, given that, because'

kiplajiz jüregijizni andan sortun ašyp baryrsiz q. k. aštyjyz qulujuz qatyna (Gen 18:5); da qylyjyz alarġa nečik jahšy körünse közlerijizd́a ančaq ošpu erenlarǵa qylmajyz neḿade anyn üčün q. k. keldilar syjynma kölegesind́a farstymnyn (Gen 19:8)

e Mod.NWKar. kačan 'when', Mod.NWKar. ki, a conjunction that introduces dependant clauses.

qadašim 'holy things, holies'

-d́an: nendij kiši urluġundan A haronnun ol bolsa cara'atly jemeśa zavly q. ašamasyn (Lev 22:4) $\Rightarrow$-tan

-ǵa: ajtqyn alarg̉a dorlaryjyzsajyn nendij kiši ki juvusa urluġujuzdan ol q. (Lev 22:3) 
-ĺarijni: ančaq q. ki bolsalar saja (Deu 12:26)

-nin: kötürsün Aharon ol jazyġyn ol q. (Exo 28:38); qyzy kohennin bolsa jat kiši artyna ol terumasyndan ol q. ašamasyn (Lev 22:12); bar terumalaryn ol q. (Num 18:19)

-tan: ötḿagin Tenrisinin qodešlarind́an ol qadašimnin da ol q. ašasyn (Lev 21:22) $\Rightarrow$-d́an

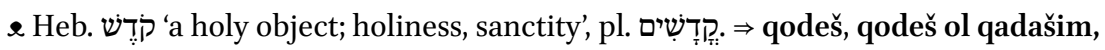
qodeš qadašim

qadeš 'temple prostitute'

bolmasyn qedeša qyzlaryndan Jisra’elnin da bolmasyn q. uvullaryndan Jisra’elnin (Deu 23:18)

\& Heb. קָדָ 'temple prostitute'.

qahal 'assembly; community'

da bilinśa ol jazyq ki jazyqly boldular anyn üčün da juvutsunlar ol q. tana balasyn syg்yrnyn hatatqa (Lev 4:14); e q. bir resim sizǵa da garipǵa ol tirilivčüǵa (Num 15:15); ajtty Mo'av qartlaryna Midjannin haligińa jalarlar ol q. ošol bar čüvrálarimizni (Num 22:4)

-ny: öltürme ošol bar ol q. ošpunu ačlyqtan (Exo 16:3); jyštyrg்anda [o]šol ol q. tartyjyz da qyčqyrmajyz (Num 10:7); jyštyrdy Moše da Aharon ošol ol q. alnynda ol skalanyn (Num 20:10); keltirmássiz ošol ol q. ošpunu (Num 20:12)

-nyn: eger bar క̌ymaty Jisra’elnin jang்ylsalar da jašyryn bolsa söz agaraqlaryndan ol q. da qylsalar birni bar micvalaryndan Adonajnyn (Lev 4:13); čygarsyn ošol ol tanany tyšqartyn avulġa da küvdürsün any ki nečik küvdürdü ošol burung் tanany hatatydy ol q. ol (Lev 4:21); bar ulusu üčün ol q. bošatlyq qolsun (Lev 16:33); qaplady alar üstüńa ol jer da taspoldular ortasyndan ol q. (Num 16:33); juvurdu ortasyna ol q. da muna bašlady ol qyranč ulusta (Num 17:13); eksilir ol žan ortasyndan ol q. ki ošol miqdašyn Adonajnyn murdar etti (Num 19:20); passim

-y: sojsunlar any bar q. క̌ymaty Jisra’elnin ol eki ingirler arasyna (Exo 12:6); bošatlyq qolsun özü üčün da üvü üčün da bar q. üčün Jisra'elnin (Lev 16:17); da neüčün ullulanasiz q. üstüńa Adonajnyn (Num 16:3)

-yjyzġa: ošol ol sözlarni ošpularny sözladi Adonaj bar q. (Deu 5:22)

-yn: nek keltirdijiz ošol q. Adonajnyn ol midbarġa (Num 20:4)

-yna: kelmesin jančylġan ajipli da kesilip tögülgen q. Adonajnyn (Deu 23:2); kelmesin mamzer q. Adonajnyn dag்yn onunču dor kelmesin anyn q. Adonajnyn (Deu 23:3); kelmesin 'Amonlu da Mo’avly q. Adonajnyn daġyn onunču dor kelmesin alarnyn q. Adonajnyn dunjaġadejin (Deu 23:4); üčünčü dor kelsin alarnyn q. Adonajnyn (Deu 23:9); Torany symarlady bizge Moše mereslik q.Ja'aqovnun (Deu 33:4)

-ynyn: tüštü Moše da Aharon jüzlari üstüna alnynda bar q. ร̌ymatynyn ulanlarynyn Jisra’elnin (Num 14:5); sözledi Moše qulaqlaryča bar q. Jisra’elnin ošol sözlerin ol širanyn (Deu 31:30)

e Heb. קָָָ 'assembly; congregation'. 
qajartyn 'whereafter, after'

keskin bellerge uturu turuvčularyn anyn da dušmanlaryn anyn q. ki tursalar (Deu 33:11)

\& Mod.NWKar. kaj-, a morpheme that forms relative pronouns + artyn 'after (postp.)'.

qamuš '1. stalk; 2. branch (of a lampstand)'

-lar: alty q. čyg்adog்anlar janlaryndan anyn (Exo 25:32); tüvme eki ol q. tübüne özünden (Exo 25:35); tüvme eki ol q. tübüne özünden (Exo 25:35); tüvme eki ol q. tübüne özünden (Exo 25:35); alty q. čygaredilerjanlaryndan (Exo 37:18); tüvme eki ol q. tübüne özünden (Exo 37:21); tüvme eki ol q. tübüne özünden (Exo 37:21); tüvme eki ol q. tübüne özünden (Exo 37:21); passim

-larğa: dajapraq alajaltysyda ol q. ol čýgučularg்a olčyraqbadan (Exo 25:33);altysyda ol q. ol čyg்uvčularg்a ol čyraqbadan (Exo 25:35); alaj altysynda ol q. ol čyg்uvčularg்a ol čyraqbadan (Exo 37:19); altysynda ol q. ol čyg்adog்anlarg்a andan (Exo 37:21)

-lary: üč q. čyraqbanyn ol janyndan (Exo 25:32); üč q. čyraqbany ol ekinči janyndan (Exo 25:32); q. alarnyn özünden bolsunlar barysy bur qaqqan aruv altyndan (Exo 25:36); ̈̈č q. čyraqbanyn ol bir janyndan (Exo 37:18); üč q. čyraqbanyn ol ekinči janyndan (Exo 37:18); tüvmeleri alarnyn da q. alarnyn özünden ediler (Exo 37:22); passim

-ta: jedi koloslar ösedilar bir q. (Gen 41:5); üč čaralar qošulġanlar ol bir q. (Exo 25:33); üč čaralar qošulġanlar ol bir q. (Exo 37:19)

-u: qylġyn čyraqba aruv altyndan qaqqan qylynsyn ol čyraqba butu anyn da q. anyn (Exo 25:31)

-un: aruv altyndan qaqqan qyldy ošol ol čyraqbany butun anyn da q. anyn (Exo 37:17)

e Mod.NWKar. kamuš 'reed', Mod.SWKar. kamus '1. reed; 2. blade (of a plant)'.

qanqajt-: bojunnu qanqajt- 'to keep the head proud'

-yp: jürüttüm sizni q. b. (Lev 26:13)

e Mod.SWKar. kankajt- 'to harden', Mod.SWKar. bojun kankajt- 'to keep the head proud (lit. to make the neck hard)'

qapaq 'covering; (?) *door wing'

-ny: da čyqty alarġa Lot ol ešikḱ da ol q. bekladi özü artyna (Gen 19:6)

e Mod.EKar. qapaq 'lid, covering'.

qaqqan 'hammered; of hammered work'

qylg̀nn eki keruvlar altyndan q. qylg்yn alarny (Exo 25:18); qylg்yn čyraqba aruv altyndan q. qylynsyn ol čyraqba (Exo 25:31); tüvmeleri alarnyn da qamušlary alarnyn özünden bolsunlar barysy bir q. aruv altyndan (Ex 25:36); qyldy eki keruvlar altyndan q. qyldy alarny (Exo 37:7); qyldy ošol ol čyraqbany aruv altyndan q. qyldy ošol ol čyraqbany (Exo 37:17); tüvmeleri alarnyn da qamušlary alarnyn özünden ediler barysy bir q. aruv altyndan (Exo 37:22); budu iši ol čyraqbanyn q. altyndan butunadejin 
japraġynadejin q. edi ol körümǵa köŕa ki körgüzdü Adonaj Mošeǵa alaj qyldy ošol ol čyraqbany (Num 8:4); qylg̀yn özüja eki byrg்ylar kümüštan q. qylg̀yn alarny (Num 10:2)

e Mod.NWKar. kah- 'to strike'.

qaraqčylyq et- 'to murder'

-megin: q.e. (Exo 20:13) $\Rightarrow$-máain

-magin: q. e. (Deu 5:17) $\Rightarrow$-megin

e Mod.NWKar. karaḥcylyh 'robbery' + aux. verb.

qarban 'offering, sacrifice'

tütetkin ošol bar ol qočqarny ol mizbeaḥta 'oladyr ol Adonajga ij qabulluq otlu q. Adonajga ol (Exo 29:18); tütetkin ol mizbeahta ol 'ola qatyna ij qabulluqqa alnynda Adonajnyn otlu q. ol Adonajġa (Exo 29:25); ošol ol ekinči qozunu qylg̀yn ol eki ingi\{r\}ler arasyna tirkisikibik ol ertenbylanyn da nesehi kibik anyn qylgyn anar ij qabulluqqa otlu q. Adonajġa (Exo 29:41); tütetme otlu q. Adonajgja (Exo 30:20); sözlegin ulanlaryna Jisra’elnin da ajtqyn alarġa nendij adam sizden ki juvunsa q. Adonajga ol tuvardan ol sygjyrdan da ol qojdan juvutujuz ošol qarbanyjyzny (Lev 1:2); passim

-dan: juvutsun andan birni bar q. teruma Adonajga (Lev 7:14)

-dy: fašmanlyq q. ol fašmanly bolma fašmanly boldu Adonajġa (Lev 5:19)

-dyr: fašmanlyq q. ol (Lev 7:5)

-ga: fašmanlyq q. (Lev 5:15); keltirsin qočqarny tüǵalni ol qojdan qyjasyjbyla fašmanlyq q. (Lev 5:18); keltirsin Adonajġa qočqarny tüǵalni ol qojdan qyjasyjbyla fašmanlyq q. ol kohenǵa (Lev 5:25); alsyn ol kohen ošol ol bir qozunu da juvutsun any fašmanlyq q. (Lev 14:12); keltirsin ošol fašmanlyq qarbanyn özünün Adonajgia ešigińa ohel mo'ednin qočqar fašmanlyq q. (Lev 19:21); passim

-lar: oldur ol q. keskin bellerge (Deu 33:11)

-larny: ört́aḿa ol q. ki mizbeaḥ üstüńa (Lev 9:23)

-lary: tütatsin ol kohen alarny ol mizbeahta otlu q. qatyna Adonajnyn (Lev 4:35); tütatsin ol mizbeahta otlu q. qatyna Adonajnyn hatatty ol (Lev 5:12)

-larymdan: berdim any otlu q. qodeš qadašimdir ol hatat kibik (Lev 6:10)

-laryn: keltirsinĺar ošol otlu q. Adonajnyn (Lev 7:30); juvutma ošol q. özlarinin alnyna Adonajnyn midbarynda Sinajnyn (Lev 7:38); jengil etḿasinlar atyn Tenrisinin özlarinin ki ošol otlu q. Adonajnyn ötḿagin Tenrisinin özlarinin alar juvutadylar da bolsunlar aziz (Lev 21:6); juvutma ošol otlu q. Adonajnyn (Lev 21:21); keltirdilar ošol q. özlarinin alnyna Adonajnyn (Num 7:3); passim

-laryndan: ol qalgan ol tirkid́an bolsun Aharonġa da uvullaryna anyn qodeš qadašim otlu q. Adonajnyn (Lev 2:3); bolsun qodeš qadašim otlu q. Adonajnyn (Lev 2:10); bar erḱk ulanlarynda Aharonnun ašasyn any ömürlük resim dorlaryjyz sajyn otlu q. Adonajnyn (Lev 6:11); budur ülüšü jag்ynmag்nnyn Aharonnun da ülüšü jag்ynmagynyn uvullarynyn otlu q. Adonajnyn (Lev 7:35); ol qalganny otlu q. Adonajnyn (Lev 10:12); passim 
-ny: sojsunlar ošol ol fašmanlyq q. (Lev 7:2)

-nyn: bošatlyq qolsun anyn üčün qočqarybyla ol fašmanlyq q. da bošatylyr jazyg்y anyn (Lev 5:16); budu üvŕatüvü ol fašmanlyq q. (Lev 7:1); alsyn ol qanyndan ol fašmanlyq q. (Lev 14:14); bersin ol kohen [...] baš barmag்y üstüna ol on jandag்y ajag்ynyn qany üstüna ol fašmanlyq q. (Lev 14:17); alsyn ol kohen ošol qozusun ol fašmanlyq q. (Lev 14:24); passim

-y: 'ola bolsa q. anyn ol syg்yrdan (Lev 1:3); eger ol qojdan bolsa q. anyn (Lev 1:9); quštan bolsa 'ola q. anyn (Lev 1:14); öźak bolsun q. anyn (Lev 2:1); eger debehasy šelamimnin bolsa q. anyn (Lev 3:1); passim

-ydy: da ašajyz any ašaġyšlyqbyla q. Pesaḥnyn ol Adonajg்a (Exo 12:11)

-yj: tava üstüńa bolsa q. öźak jumurulgan javbyla maca bolsun (Lev 2:5); eger tirki išlanǵan panv́ada bolsa q. öźak javbyla qylynsyn (Lev 2:7); bar q. üstüńa juvutqun tuz (Lev 2:13)

-yjyzny: sözlegin ulanlaryna Jisra’elnin da ajtqyn alarġa nendij adam sizden ki juvunsa qarban Adonajġa ol tuvardan ol syġyrdan da ol qojdan juvutujuz ošol q. (Lev 1:2)

-ym:ajtqyn alarġa ošol qarbanymny ötḿagimni otlu q. byla ij qabulluqlarymny saqlajyz juvutma maja vaġdasynda (Num 28:2)

-ymny: ajtqyn alarġa ošol q. ötḿagimni otlu qarbanlarymbyla ij qabulluqlarymny saqlajyz juvutma maja vaġdasynda (Num 28:2)

-yn: sojujuz q. ol Pesạ̣nyn (Exo 12:21); da klese qylma q. Pesạ̣nyn Adonajga kereklidi hatna qylma anar (Exo 12:48); juvutsun ol bedenelerden jemese ol kögürčün balalaryndan ošol q. özünün (Lev 1:14); q. ilknin juvutujuz alarny Adonajġa (Lev 2:12); bar q. tirkijnin (Lev 2:13); passim

-ynyn: debehasydy q. Pesaḥnyn ol Adonajġa (Exo 12:27); budur resimi q. ol Pesahnnyn heč jat kiši ašamasyn andan (Exo 12:43); sunsun ošol qolun bašy üstüńa q. da sojsun any ešigind́a ohel mo'ednin (Lev 3:2); sunsun ošol qolun bašy üstüna q. da sojsun any alnynda ohel mo'ednin (Lev 3:8); sunsun ošol qolun bašy üstüńa q. da sojsun any alnynda ohel mo'ednin (Lev 3:13); passim

\& Heb. קָרְבָ 'offering, sacrifice, oblation'.

qara budaj $\Rightarrow$ budaj

qararmaq 'lit. blackening; a kind of disease, probably gangrene'

urar seni Adonaj suvuq hastalyq byla da issi hastalyq byla da küvmek byla da q. byla da qurumaq byla da sarylyqbyla da ješillikbyla da quvarlar seni taspolganyjadejin (Deu 28:22)

\& Mod.SWKar. kararmak 'blackening', used for BHeb. חִרְוּר 'violent heat, fever', see Klein (1987: 232), Koehler \& Baumgartner (1985: 332-333). Cf. also Mod.Heb. חַרְרחר 'gangrene, plough-staff'. Given that symptoms of gangrene may include a change in skin colour to black, we follow here the interpretation proposed in Jankowski \& Aqtay \& Cegiołka \& Çulha \& Németh (2019: II 266), where EKar. harhur is translated 'gangrene'. 
qaršy: qaršysyna köŕa 'corresponding to'

qylajym anar bolušluq q. k. (Gen 2:18); Adam özüńa tapmady bolušluq q. k. (Gen 2:20)

e Mod.NWKar. karšy 'opposite', Mod.NWKar. koŕa 'according to (postp.)'.

qasja 'cassia'

q. beš jüz ol aziz mitqal byla (Exo 30:24)

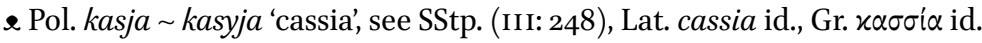

qaty: qaty enseli 'stiff-necked, obstinate'

ajtqyn uvullaryna Jisra'elnin siz ulus q. e. (Exo 33:5)

-dir: muna ulus q. ensel[i] dir ol (Exo 32:9); ki ulus q. ensel[i]dir (Exo 33:3)

^ Mod.NWKar. katy 'hard', Mod.NWKar. eńśa 'back of one's head' + deriv. suff.

qaty: qaty juqu 'deep sleep'

saldy Adonaj Tenri q.j. ol Adam üstüna (Gen 2:21); q.j. tüštü Avram üstüne (Gen 15:12)

e Mod.NWKar. katy 'hard', Mod.NWKar. juku 'dream'.

qatyr *'male donkey'

-lar: teveler ömüzdüredog்anlar balalarybyla otuz tiši tanalar qyrq da erkek tanalar on ešeklar egirmi da q. on (Gen 32:16)

e Mod.NWKar. katyr 'mule'; the meaning is reconstructed based on the context.

qatyš 'wild'

da dag்yn q. ol köbüsü bardy birgelerińa alarnyn da qoj da syg்yr tuvar küčlü astry (Exo 12:38); da ol q. el ki ortasynda anyn (Num 11:4)

e Mod.SWKar. katys 'wild'. Interpreting Mod.SWKar. katys kijik as 'a kind of fly' seems to be an error that entered KarRPS (s.v. катьı).

qazan 'cauldron'

olturg்anymyzda ol q. et qatyna ašag்anymyzda ötmek (Exo 16:3)

-laryn: qyldy ošol bar savutlaryn ol mizbeahnyyn ošol ol q. (Exo 38:3)

e Mod.SWKar. kazan 'cauldron'.

qereạ 'bald'

kiši ki julqunsa bašy anyn q. ünd́áladi ol aruvdu ol (Lev 13:40)

e Heb. קָרֵ 'bald'.

qešita 'qesitah (old weight of unknown value)'

-ǵa: satyn aldy ošol ülüšün ol tüznün ki qurdu anda čatyryn qolundan ulanlarynyn Hamornun atasynyn Šehemnin jüz $\langle q\rangle.\{$ qozuġa $\}$ (Gen 31:19)

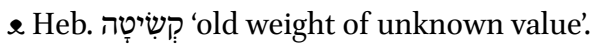

qobuluš- 'to be confused'

-adylar: ajtyr par'o ulanlary üčün Jisra’elnin q. alar jerde (Exo 14:3)

e Mod.NWKar. kobulušmah ‘muddle, confusion', Mod.SWKar. kobulus- 'to cause confusion'.

qodeš '1. holy; 2. sanctity'

ajyrsyn sizge ol parohet arasyna ol qodešnin da arasyna q. ol qodešnin (Exo 26:33); qyl-

g̀yn čalma aruv altyndan da tužurg̉un anyn üstüne tužurmaqlary mohornun q. la-H 
(Exo 28:36); da bolsunolmizbeaḥq. qadašim (Exo 29:37); bošatlyq qolsun anyn üstüne dorlaryjyz sajyn q. qadašim ol Adonajg̉a (Exo 30:10); aziz etkin alarny da bolsunlar q. qadašim bar ol tijüvčü alarga kereklidi ki azizlengej (Exo 30:29); passim -ind́a: bergin ošol ol kaporetni aronu üstüne ol šarajatnyn q. ol qodešnin (Exo 26: 34)

-ke: kelgeninde ol q. saġynčlyqqa alnynda Adonajnyn hammeše (Exo 28:29); ešitilsin avazy anyn kelgeninde ol q. alnyna Adonajnyn (Exo 28:35); ošol ol jaġynmaq javny da ošol tütüsün ol ot $\{j\}$ amlarnyn q. barča nečik ki bujurdum saja alaj qylsynlar (Exo 31:11) $\Rightarrow$-ḱa

-ka: muna kijirilmáadi ošol qany anyn ol q. ičkariǵa (Lev 10:18); kelmasin har vahtta ol q. ičkartin (Lev 16:2); kelsin Aharon ol q. qany byla tananyn (Lev 16:3); aq upraqlarny ki kijdi kelǵanind́a ol q. (Lev 16:23); juvuġunda ulanlary Jisra’elnin ol q. (Num 8:19) $\Rightarrow$ -ke

-lerinin: ki qodeš etseler ulanlary Jisra’elnin bar berneleri sajyn q. (Exo 28:38)

-lari: bar teruma bar q. sajyn ulanlarynyn Jisra’elnin (Num 5:9); berdim saja ošol saqlavun terumalarymnyn bar q. sajyn ulanlarynyn Jisra'elnin (Num 18:8)

-ĺarin: jengil etḿasinlar ošol q. ulanlarynyn Jisra’elnin (Lev 22:15); kötürsünlar özlari özlarińa క̌urumun fašmanlyqnyn ašaġanlarynda ošol q. (Lev 22:16); q. ulanlarynyn Jisra'elnin (Num 18:32)

-larind́an: క̌an ki tansa tanmaq da jazyqly bolsa janġylyšlyqbyla q. Adonajnyn (Lev 5:15); ötḿagin Tenrisinin q. ol qadašimnin (Exo 21:22); ajyrylsynlar q. ulanlarynyn Jisra'elnin (Lev 22:1)

-ni: heč kiši ašamasyn q. očary kohennin da jalčy ašamasyn q. (Lev 22:10); kiši ki ašasa qodešni (Lev 22:14); bersin kohenǵa ošol ol q. (Lev 22:14); qaplama ošol ol q. (Num 4:15); kelḿasinlar baqma taġatqanda ošol ol q. da ölarlar (Num 4:20); passim

-nin: ajyrsyn sizge ol parohet arasyna ol q. da arasyna qodeš ol q. (Exo 26:33); bergin ošol ol kaporetni aronu üstüne ol šarajatnyn qodešind́a ol q. (Exo 26:34); mitqaly byla ol q. (Exo 30:13); bar išin qulluġunun ol q. (Exo 36:1); atyn qylmasynlar artyq iš terumasyna ol q. (Exo 36:6); passim

-te: jumuš etŕa q. (Exo 28:43); kelir ohel mo'edge jumuš etŕa q. (Exo 29:30); bojavly upraqlarny jumuš etŕa q. (Exo 35:18); qyldylar bojavly upraqlar jumuš etme q. (Exo 39:1); bojavly upraqlarny jumuš etŕa $q$. (Exo 39:41); passim $\Rightarrow$-ta

-ti: sunsun alarny ol kohen tenufa alnynda Adonajnyn q. ol (Num 6:20)

-ta: q. ašalmasyn otta küvdürülsün (Lev 6:23); keŕakli edi ki ašama ašag்ajsiz any q. ki nečik bujurdum (Lev 10:18); bird́a adam bolmasyn ohel mo'edd́a kelǵanind́a bošatlyq qolma q. čyqqanynadejin (Lev 16:17); bošatlyq qolma q. (Lev 16:27); jumuš etadilar alar byla q. (Num 4:12); passim $\Rightarrow$-te

-tan: ošol neki jazyqly boldu ol q. tölasin (Lev 5:16)

\& Heb. קדָָש 'a holy object; holiness, sanctity'. $\Rightarrow$ qadašim, qodeš et-, qodeš ol qadašim, qodeš qadašim 
qodeš et- 'to sanctify, to consecrate'

-ḱan: kiši ošol q. e. nerśalari anyn (Num 5:10)

-masin: q. e. kiši any (Lev 27:26)

-seler: bolsun manlajy üstüne Aharonnun da kötürsün Aharon ol jazyg்yn ol qadašimnin ki q. e. ulanlary Jisra'elnin (Exo 28:38)

-śa: eger tüzünd́an tutuvluginun q. e. kiši (Lev 27:16); eger jylyndan ol jovelnin q. e. tüzün özünün qyjasyja köra e kohen qajjam bolsun (Lev 27:17); eger ol joveld́an sortun q. e. tüzün özünün (Lev 27:18); q. e. kiši Adonajga (Lev 27:22)

-üvčü: eger juluma julusa ošol ol tüznü ol q. e. (Lev 27:19)

\& Heb. קדָש 'a holy object; holiness, sanctity' + Kar. aux. verb. $\Rightarrow$ qodeš

qodeš ol qadašim 'holy of holies'

budur qulluġu uvullarynyn Qahatnyn ohel mo'edd́a q. o. q. (Num 4:4)

-ǵa: ölḿasinlar juvuǵanlarynda q. o. q. Aharon da uvullary (Num 4:19)

-nin: ötmiagin Tenrisinin qodešlarind́an o. q. (Lev 21:22); bu bolsun saja qodešindáan o.

q. (Num 18:9)

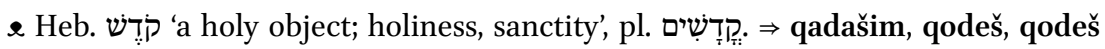
qadašim

qodeš qadašim 'holy of holies'

da bolsun ol mizbeah q. q. (Exo 29:37); bošatlyq qolsun anyn üstüne dorlaryjyz sajyn q. q. ol Adonajġa (Exo 30:10); aziz etkin alarny da bolsunlar q. q. bar ol tijüvčü alarġa kereklidi ki azizlengej (Exo 30:29); hadir bolur šehinam aškara bolma saja anda q. q. bolsun sizge (Exo 30:36)

-di: q. q. ol (Lev 6:18); budu üvŕatüvü ol fašmanlyq qarbannyn q. q. ol (Lev 7:1); ašajyz any macalar janynda ol mizbeahnyn ki q. q. ol (Lev 10:12); ol kohenǵadi q. q. ol (Lev 14:13); q. q. saja ol da uvullaryja (Num 18:9); passim

-dir: q. q. ol hatat kibik (Lev 6:10); erḱkak kohenlard́a ašasyn any q. q. ol (Lev 6:22); aziz orunda ašalsyn q. q. ol (Lev 7:6); aziz orunda ki q. q. ol (Lev 10:17); aziz orunda ki q. q. ol (Lev 24:9); passim

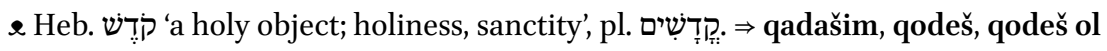
qadašim

qoj 'flock'

-dan: ajtty men ijermen ulaġyn ečkilernin ol q. (Gen 38:17); keltirsin ošol fašmanly qarbanyn Adonajġa jazyg்y üčün ki jazyqly boldu tišinin ol q. qozunu jemeśa ulaġyn ečkilarnin ḥatatqa (Lev 5:6); keltirsin ošol fašmanly qarbanyn Adonajg̉a qočarny tüǵalni ol q. (Lev 5:15); keltirsin qočqarny tüǵalni ol q. qyjasyjbyla fašmanlyq qarbanġa ol kohenǵa (Lev 5:18); keltirsin Adonajġa qočqarny tüǵalni ol q. qyjasyjbyla fašmanlyq qarbanġa ol kohenǵa (Lev 5:25)

-ġa: barg்yn endi ol q. da alġyn maja andan eki ulaqlaryn ečkilernin (Gen 27:9)

-nun: turg்uzdu Avraham ošol jedi qozularyn ol q. jalg̀yzlarny (Gen 21:28)

e Mod.NWKar. koj 'sheep'. 
qoj- 'to make'

-armen: q. any ullu hanlyqqa (Gen 17:20); ki ullu hanlyqqa q. any (Gen 21:18); ki ullu hanlyqqa q. seni (Gen 46:3); da q. ošol urluġujnu qumun kibik ol tengiznin ki sanlamastyköplükt́an (Exo 32:12);

-du: kim q. avuz adamġa jemese kim q. tilsizni jemese kim q. sandravnu (Exo 4:11); da q. ošol ol tengizni quruġa andan sortun (Exo 14:21)

-dular: ki q. any jumuš etüvčü uvullaryna Bilhanyn da Zilpanyn (Exo 37:2)

e ModNWKar. koj- 'to put', a calque of Heb. נָזֵז '1. to give; 2. to permit; 3 . to deliver; 4. to put, to set; 5 . to make'.

qol: qolun toltur- 'to ordain'

-gun: jag்yndyrg்yn alarny da t. ošol q. alarnyn da aziz etkin alarny da kohenlik etsinler maja (Exo 28:41); bolsun alarġa kohenlik ömürlük resimge da t. q. Aharonnun da qolun avullarynyn (Exo 29:9); jedi künler t. ošol q. alarnyn (Exo 29:35)

-ma: t. alarbyla ošol q. alarnyn (Exo 29:29)

-sa: bošatlyq qolsun ol kohen ki jag்yndyrsa any da ki t. ošol q. anyn kohenlik etŕa (Lev 16:32); t. ošol q. anyn kijmía ošol ol upraqlarny (Lev 21:10)

-ujuz: t. qolujuznu bügün jumuš etme Adonajga (Exo 32:29)

-ur: jedi künlar t. ošol qollaryjyzny (Lev 8:33)

e Mod.NWKar. kol 'hand', Mod.NWKar. toltur- 'to fill'.

qomuz '1. creeping animal; 2. living thing'

čygarsyn ol jer [tiri] క̌an ̌̌ynsy sajyn tuvarda q. da kijigin jernin (Gen 1:24); bar ol kijik క̌ynsysajyn da bar ol tuvar ふ̌ynsysajyn da bar ol q. ol tebrenedoġan ol jer üstüńa 3̌ynsy[s]ajyn da bar ol quš žynsysajyn (Gen 7:14); qušta da tuvarda da bar ol q. da ol tebrenedoġan ol jer üstüna (Gen 8:17); bar ol kijik bar ol q. da bar ol quš bar tebrenedoġan ol jer üstüna uruvlarysajyn čyqtylar ol gerepd́an (Gen 8:19); bar q. ki ol tiridi sizǵa bolsun [jem] ǵa ješilliginkibik kögötnün berdim sizǵa ošol [bary]syn (Gen 9:3); passim

-da: da bar ol q. ol tebrenedogian ol jer üstüna (Gen 1:26); bar ten ol tebrenüvčü ol jer üstüńa qušta da tuvarda da kijikt́a da bar ol q. da ol qozlajdogan ol jer üstüńa da bar ol adam (Gen 7:21)

-gaa: adamdan tuvarġadejin q. dejin da qušunadejin (Gen 7:23); kiši ki tijśa nendijd́a q. ki murdar bolady anynbyla (Lev 22:5)

-ndan: quštan گ̌y [nsy] sajyn da ol tuvardan گ̌ynsysajyn bar q. ol jernin žynsysajyn ekśari baryndan (Gen 6:20)

-un: ošol ki [jigin ol] jernin žynsy sajyn da ošol ol tuvarny žynsy sajyn da o[šol ol bar] q. ol jernin گ̌ynsy sajyn (Gen 1:25)

\& Mod.NWKar. komuz 'insect'. $\Rightarrow$ qumuz

qozla- 'to creep'

-jdogan: bar ol qomuzda ol q. ol jer üstüńa (Gen 7:21)

e Mod.EKar. qozala- 'to creep, to crawl', Mod.NWKar. kozla- 'to produce, to sire, to breed'. $\Rightarrow$ qozlamaq 
qozlama $\mid \mathbf{q}$ *'swarm'

-|'̇yn: qozlasynlar ol suvlar q. tiri žannyn (Gen 1:20)

e Mod.EKar. qozala- 'to creep, to crawl', Mod.NWKar. kozla- 'to produce, to sire, to breed'. $\Rightarrow$ qozla-

qozu 'ewe'

-lar: ečkilar eki jüz da tegelar egirmi q. eki jüz da qočqarlar egirmi (Gen 32:15)

-laryj: q. da ečkilerij tuv etmedilar (Gen 31:38)

e Mod.NWKar. kozu 'lamb'.

quگ̌ur 'leaven'

jedi künler q. tabulmasyn üvlerijizd́a (Exo 12:19); körünmesin saja q. bar čegïje (Exo 13:7); heč q. da heč čyjbal tütatḿajiz (Lev 2:11); q. ötmaknin juvutsun (Lev 7:13); ašamag்yn anyn qatyna q. ötmek jedi künler (Deu 16:3)

-nu: jedi künler macalar ašajyz tek burun ol burunġu künden eksitijiz q. üvlerijizden (Exo 12:15)

\& Mod.NWKar. kuక̌ur 'sour'.

qumuz '1. creeping animal; 2. living thing'

budur sizǵa ol murdar q. da ol tebŕańadogian ol jer üstüna ol holed da ol 'ahbar da ol cav žynsysajyn (Lev 11:29); bulardylar ol murdarlar sizǵa bar ol q. da bar ol tijüvčü alarġa ölǵanlarind́an sortun murdar bolsun ol ingirǵadejin (Lev 11:31); bar ol q. ol tebŕańadoġan ol jer üstüńa qusqunčudu ol ašalmasyn (Lev 11:41); jiŕanči etŕajiz ošol ̌̌anlaryjyzny bar ol q. byla ol tebŕanadoġan da murdar bolunmajyz alarbyla (Lev 11:43); menmen Adonaj Tenrijiz da azizlanijiz da bolujuz azizlar ki azizdir men da murdar etḿajiz ošol ร̌anlaryjyzny bar ol q. byla ol tebŕanüvčünü ol jer üstüńa (Lev 11:44)

-nu: bar jürüvčünü qaryn üstüna da bar jürüvčü dört ajaq üstüńa bar köp ajaqlygadejin bar ol q. ol tebranüvčünü ol jer üstüna ašamajyz alarny ki qusqunčudu alar (Lev 11:42)

-nun: jemeśa žan ki tijśa nendijd́a murdar nerśaǵa jemeśa gövd́asińa murdar kijiknin jemeśa gövd́asińa murdar tuvarnyn jemeśa gövd́asińa murdar q. da jašyryn bolsa andan da ol murdar boldu da fašmanly bolsa (Lev 5:2)

-u: bar q. ol qušnun ol jürüvčü dörtajaq üstüna qusqunčudu ol sizǵa (Lev 11:2o); bar q. ol qušnun ki anar dört ajaqlar qusqunčudu ol sizǵa (Lev 11:23)

-undan: barča neki joḥtur anar qanat da qabuq suvlarda da öźanlard́a bar q. ol suvlarnyn da bar ol tiri žandan ki suvlarda qusqunčudu alar sizǵa (Lev 11:10); tek ošol bunu ašajyz bar q. ol qušnun ol jürüvčünün dört ajaq üstüna ki anar tizlar joġarraq ajaqlaryndan sekirmáa alarbyla ol jer üstüńa (Lev 11:21)

e Mod.NWKar. komuz 'insect'. $\Rightarrow$ qomuz

qungurav 'bell'

-lar: da altyn q. ortalarynda alarnyn (Exo 28:33)

e Mod.NWKar. konhurov kunhurov 'bell'. 
*qurtlan- 'to become maggot infested'

-dy: tynlamadylar Mošege da qaldyrdylar andan erenler tanġadejin da q. da boldu qurtlar andan sortun nečik sasydy da ačuvlandy alar üstüne Moše (Exo 16:20)

e Mod.SWKar. kurtaj- 'to become maggot infested', cf. EKar. qurtla- id. in C (Exo $16: 20)$.

quršanġy *'swollen'

jemeśa bükrü jemeśa uvaq jemeśa belma közünd́a jemeśa q. jemeśa qynġyrajġan jemeśa šiškan ajipli (Lev 21:20)

-ny: soqurnu jemeśa syng்anny jemeśa kesilǵanni jemeśa belmalyny jemeśa q. jemeśa qyng்yrajġanny juvutmajyz bularny Adonajġa da otlu qarban bermejiz alardan ol mizbeaḥüstüńa Adonajġa (Lev 22:22)

e Mod.NWKar. kurša- 'to make circles', in Lev 21:20 and Lev 22:22 it renders BHeb.

גָּר :eczema and swell of the skin', see Koehler \& Baumgartner (1985: 192).

qusqunču 'detestable'

da q. bolsunlar sizǵa etlarind́an alarnyn ašamajyz (Lev 11:11)

-du: bar qumuzundan ol suvlarnyn da bar ol tiri ̌̌andan ki suvlarda q. alar sizǵa (Lev 11:10); barča neki johtu anar qanat da qabuq suvlarda q. ol sizǵa (Lev 11:12); bar qumuzu ol qušnun ol jürüvčü dörtajaq üstüna q. ol sizǵa (Lev 11:20); bar qumuzu ol qušnun ki anar dört ajaqlar q. ol sizǵa (Lev 11:23); bar ol qumuz ol tebrańadog்an ol jer üstüńa q. ol ašalmasyn (Lev 11:41)

-dur: ošol bunu jiŕanči etijiz ol quštan ašalmasynlar q. alar (Lev 11:13)

\& Mod.SWKar. kuskuncu 'disgusting, detestable'.

quš 'flying creature'

-nun: bar qumuzu ol q. ol jürüvčü dörtajaq üstüńa qusqunčudu ol sizǵa (Lev 11:20); bar qumuzundan ol q. oljürüvčünün dörtajaq üstüna kianar tizlarjoġarraq ajaqlaryndan sekirḿa alarbyla oljer üstüńa (Lev 11:21); bar qumuzu ol q. ki anar dört ajaqlar qusqunčudu ol sizǵa (Lev 11:23); bar qomuzu ol q. murdardy ol sizge ašalmasynlar (Deu 14:19)

e Mod.NWKar. kuš 'bird', cf. BHeb. עוֹ '1. fowl, bird; 2. flying creature: fowl, insects'.

qyl- 'to work'

-armen: da haligine qačan q. dag்yn mend́a üvüme (Gen 30:30)

e Mod.NWKar. kyl- 'to do', cf. Heb. עָשָָׁ' '1. to do, to make; 2. to work; 3. to act'.

qyng.gr et- 'to convict, to find guilty'

-seler: ki q. e. törečiler eki anča tölesin dostuna (Exo 22:8); ki bolsa talaš arasyna erenlernin da juvusalar ol törege da töre etseler alarny da rast etseler ošol ol rastny da q.e. ošol ol qyng்yrny (Deu 25:1)

e Mod.NWKar. kynhyr 'crooked', Mod.EKar. qynġyr et- 'to harm'.

qypčy- 'to cut out, to cut in pieces'

-dy: juġarttylar ošol ol altyn blehalarny da q. šnurlar qylma ortasynda ol köknün da ortasynda jipkinnin da ortasynda ol qyrmyzy jipeknin da ortasynda ol b[e]znin iši ojuvlavčunun (Exo 39:3) 
e Mod.SWKar. kypty- 'to cut' (kyp - + ty-, for this etymology, see A. Zajączkowski 1932: 86), cf. Mod.NWKar. kyptylar 'scissors'. Cf also Tkc. kypty- 'to cut' (ÈSTJa 200o: 225226), and Kirg. qypčy- 'to cut, to trim; to press', see Radlov (1899: II/1: 845, s.v. kыпиць), and Judachin (1965:492). Cf. SWKar. qypcy- used in ADub.III.82 (Exo 39:3), JSul.III.o1 (Deu 25:12), and TKow.01 (207 ro; 2 Chr 28:24) in the meaning of 'to cut (in pieces)'. In other manuscripts the equivalent of qypcy y- in Exo 39:2 is kes- 'to cut', cf. kesti (in H, C, M, and E) and kestilär (in Evr I Bibl 143). In ADub.III.73, the equivalent of qypcyused in JSul.III.o1 in verse Deu 25:12 is kes- 'to cut'. This meaning fits in well with the context.

qyzuv *'reddening; burn'

-u: eger ornunda tursa ol temgil jajylmasa q. ol čybannyn ol da aruv etsin any ol kohen (Lev 13:23)

-udu: eger ornunda tursa ol temgil jajylmady terida da ol tundu küvmagidi ol küvḿaknin ol da aruv etsin any ol kohen q. ol küvḿaknin ol (Lev 13:28)

e Mod.NWKar. kyz- 'to burn, to redden' + deriv. suff.

$\mathbf{R}$

ra'a 'a bird of prey; the red kite (?)'

-ny: ol $r$. da ošol ol ajany da ol dajany žynsysajyn (Deu 14:13)

e Heb. רָָָ 'a bird of prey, probably the red kite', see Klein (1987: 6oo), Koehler \&

Baumgartner (1985: 864). $\Rightarrow$ da’a

raḥam 'carrion vulture'

-ny: ošol ol tinšemetni da ošol ol qa’atny da ošol ol r. (Lev 11:18);

e Heb. רחָָ 'carrion vulture'. $\Rightarrow$ raḥama

raḥama 'carrion vulture'

-ny: ol qa’a[t]ny da ošol $r$. da ošol ol šalahny (Deu 14:17)

e Heb. רָחָָָ 'carrion vulture'. $\Rightarrow$ raḥam

raqia' 'firmament'

-syna: qu[š učqa]lasyn ol jer üstüna juvuq r. ol kökler[nin] (Gen 1:20)

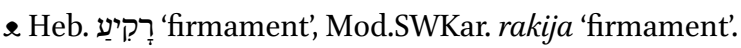

revent *'willowherb'

ajtty Adonaj Mošege alġyn özüje otjamlar mušk da r. da galban otjamlar da aruv levona bašqa bašqa har birisi bolsun (Exo 30:34)

e Perhaps related to Russ. dial. ревенка 'willowherb (Chamaenerion angustifolium)' SRNG (XXXIV: 367 ). In the translation, the word is used to render Heb. שִִׁחלֶת 'onycha' which is associated with (and perhaps etymologically related to) the Heb. root שחל 'to roar' and with שِ שַח 'lion' (Klein 1987:650). The reason behind such a Karaim translation might stem from the fact that Russ. dial. ревенка is, in turn, associated with Russ. реветь 'to roar'. Cf. SWKar. revend 'willowherb' in H (Exo 30:34) and šehelet 'onycha' in C (Exo 30:34). 


\section{rubin 'ruby'}

-nin: dört jergeler taštan jergesin r. smaraknyn da karbankulisnin ol bir jerge (Exo 28:17); jergesi r. šmaraknyn da karbankulisnin (Exo 39:10)

\& Pol. rubin 'ruby', Russ. рубин id.

\section{S}

sa'a 'seah, a dry and liquid measure'

-lar: ̌̌ahtlaġyn üčs. un özek basqyn da qylġyn jajmalar (Gen 18:6)

\& Heb. סָזָ 'seah, i.e. a dry and liquid measure, one third of an ephah'.

safir 'sapphire'

išikibik kirpičnin išlengen [bö]lörü byla s. tašynyn (Exo 24:10); ol ekinči jerge sürne tašy s. tašy da dijament (Exo 28:18); ol ekinči jerge sürne tašy s. tašy da dijament (Exo 39:11)

\& Heb. סַפִּיר 'sapphire'.

sa|k 'amout'

-|hyn: hešbonetsin anar ol kohen ošol s. ol qyjasyjnyn jylynadejin ol jovelnin da bersin ošol ol qyjasyjnyn ol künd́a qodeš Adonajġa (Lev 27:23)

\& Heb. דסָ 'amount', Mod.SWKar. sak 'amount', see Németh (2011b: 315).

sal'am 'a kind of locust; bald locust (?)'

-ny: ošol bunlary alardan ašajyz ošol ol arbeni ̌̌ynsysajyn da ošol s. ̌̌ynsysajyn da ošol ol ḥargolnu žynsysajyn ošol ol hagavny žynsysajyn (Lev 11:22)

\& Heb. סלְָָ 'a kind of locust', see Klein (1987: 448), 'edible locust' Koehler \& Baumgartner (1985:66o)

sanyn al- 'to take the sum of'

-g̀yn: a. ošol s. atlarynyn (Num 3:40); a. ošol s. bašynyn uvullarynyn Geršonnun (Num 4:22)

-mag்yn: ošol s. bašlarynyn a. (Num 1:49)

-yjyz: a. s. bašynyn bar ̌̌ymatynyn ulanlarynyn Jisra’elnin (Num 1:2)

\& Mod.NWKar. san 'number, count', Mod.NWKar. al- 'to take'.

sanly *few in number'

da men s. el (Gen 34:30); boluredi ol bulut s. künlar ol miškan üstüna (Num 9:20); tozdurur Adonaj sizni uluslarda da qalyrsiz s. el hanlyqlarda (Deu 4:27); bolgaj eli anyn s. sanlary arasyna ševetlerinin Jisra’elnin (Deu 33:6)

$\iota$ Mod.NWKar. san 'number' + deriv. suff. The meaning is reconstructed based on the context.

sargy postp. '-ward, in the direction of'

ol ullu özenden özeninden Peratnyn da ol máarav s. tengizgedejin bolur čegijiz (Deu 11:24); bar jerin Juhudanyn ol ma'arav s. tengizge dejin (Deu 34:2)

e Mod.NWKar. sary '-ward, in the direction of (postp.)'. 
saruv́ak *'adder'

-li (deriv. suff.): jürütüvčünü seni midbarda ol ullu da ol qorqunčlu küvdürgüč jylanly da s. da suvsaply ki joġedi suv ol čyg்aruvču saja suv almas skaladan (Deu 8:15)

e Kmk. sariek 'adder', Krč. sarujbek saryjek 'crocodile', see KumRussS (1969: 279), Kar-BalkRussS (1989: 543), and Menges (1979: 172). Cf. SWKar. saruvek *'adder' in H (Deu 8:15), and TKow.o2 (Judges 8:7).

sarylyq 'a kind of fungal disease, probably blight (lit. yellowness)'

urar seni Adonaj suvuq hastalyq byla da issi hastalyq byla da küvmek byla da qararmaq byla da qurumaq byla da s. byla da ješillikbyla da quvarlar seni taspolġanyjadejin (Deu 28:22)

e Mod.NWKar. sarylyh '1. yellowness; 2. jaundice'; a translation of BHeb. 1' שדָדפוֹן blight; 2. scorching', see Klein (1987: 642), Koehler \& Baumgartner (1985: 950), but cf. also Mod.Heb. שִדּפוֹן '1. blight, blast; 2. fungal disease of corn; 3. emptiness, hollowness' (Comey \& Tzur 2006: 616), and Heb. שדף 'to blast, to blight, to scorch' (Klein 1987: 642; Koehler \& Baumgartner 1985: 950). As far as the reconstruction of the word's meaning is concerned, cf. the symptoms of blight that may include sudden and severe yellowing of leaves.

savut 'item, thing; equipment'

bar ol s. ki oltursa anyn üstüna murdar bolsun (Lev 15:26); keltirdiler halha da syrg்a da jüzük da pižma bar altyn s. (Exo 35:22)

-lary: da beklüvüčlari anyn da baġanalary anyn da tabanlary anyn da bar s. anyn da bar qullug்u anyn (Num 3:36); baġanalary ol qahranyn čüvŕa da tabanlary alarnyn da qazyqlary da šnurlary alarnyn bar s. sajyn da bar qulluqlarysajyn alarnyn da atlarbyla symarlajyz ošol savutlaryn saqlavynyn jüklarinin (Num 4:32)

-laryn: bersinlar any da ošol bar s. anyn qaplavġa tahaš terisind́an da bersinlar ol üśak üstüna (Num 4:10); bersinlar anyn üstüńa ošol bar s. anyn ki jumuš etadilar anyn üstüńa alar byla (Num 4:14); bersinlar anyn üstüńa ošol bar s. anyn ki jumuš etadilar anyn üstüna alar byla ošol mačmarlarny da ošol oljyrġaqlarny da ošol ol sibirtkilarni da ošol ol kuboklarny bar s. ol mizbeahnyn da jajsynlar anyn üstüńa qaplav (Num 4:14); nečik tüǵallaśa Aharon da uvullary anyn qaplama ošol ol qodešni da ošol bar s. ol qodešnin (Num 4:15); ošol enlarin ol qaḥranyn da ošol qaplavyn ešiginin qabaġyny ol qaḥranyn ki ol miškan qatyna da ol mizbeaḥ qatyna čüvŕa da ošol šnurlaryn alarnyn da ošol bar s. qulluqlarynyn da ošol barča neki qylyndy alarġa da qulluq etsinlar (Num 4:26); baġanalary ol qahranyn čüvŕa da tabanlary alarnyn da qazyqlary da šnurlary alarnyn bar savutlarysajyn da bar qulluqlarysajyn alarnyn da atlarbyla symarlajyz ošol s. saqlavynyn jüklarinin (Num 4:32); edi tüǵallaǵan künd́a Moše turguzma ošol ol miškanny da jagjyndyrdy any da aziz etti any da ošol bar s. da ošol ol mizea hny da ošol bar savutlaryn anyn da jaġyndyrdy alarny da aziz etti alarny (Num $7: 1)$

-qa: bar ol tijüvčü bar s. ki oltursa anyn üstüńa (Lev 15:22) 
-ta: jemeśa osnovada jemeśa utokta üskülünün jemeśa junnun jemeśa terid́a jemeśa nendijd́a terili s. (Lev 13:48); bolsa ol hastalyq jašyl syman jemeśa qyzyl syman upraqta jemeśa terid́a jemeśa osnovada jemeśa utokta jemeśa nendijd́a terili s. hastalygjydy cara'atnyn ol da körgüzülsün ol kohenǵa (Lev 13:49); bar ol töśak ki jatsa anyn üstüna ol zavly murdar bolsun da bar ol s. ki oltursa anyn üstüna murdar bolsun (Lev 15:4)

e Mod.NWKar. savut '1. vessel, dish; 2. weapon'.

savuqma|q 'efflorescence'

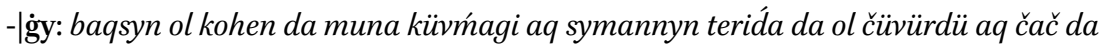
s. čyj etnin küvmakte (Lev 13:10); guf ki bolsa terisind́a anyn küvḿagi otnun da bolsa s. ol küvḿaknin aq temgil qyzyl syman jemeśa aq syman (Lev 13:24)

e Mod.NWKar. savuh- 'to recover', Mod.EKar. savuq- '1. to recover, to be healthy; 2. to bloom'.

sefer '1. book; 2. scroll'

olturg்anyndačoq bijlik tahty üstüne da jazsyn özüne ošol mišne ol Torany ošpunu s. üstüne alnyndan ol kohenlernin ol Levililernin (Deu 17:18); barda hastalyqny da bar karanjany kijazylmaġandy s. ol Torada ošpu keltirir alarny Adonaj üstüje (Deu 28:61); tynlasajününe Adonaj Tenrijnin saqlama micvalaryn anyn da resimlerin anyn oljazylganny s. ol Tora (Deu 30:10); edi tügellegečoq Moše jazma ošol sözlerin ol Toranyn ošpunun s. üstüne tügellengenlerinedejin (Deu 31:24); alma ošol s. ol Torany ošpunu da qojujuz any janyndan aron šertinin Adonajnyn (Deu 31:26)

-de: tynlamasaj qylma ošol bar sözlerin ol Toranyn ošpunun ol jazylganlarny ošpu s. qorqma šeminden ol Tenrinin (Deu 28:58); jatyr anda bar ol qarg்yš ol jazylg்an ošpu s. (Deu 29:19); jazylġan ošpu \{ol T [ora]da ol\} s. (Deu 29:20); qabundu ačuvu Adonajnyn ol jer üstüne keltirme anyn üstüne ošol bar ol qarg்yšny ol jazylġanny ošpu s. (Deu 29:26)

-i: budur s. tuvmušlarynyn Adamnyn (Gen 5:1)

-in: aldy ošol s. ol šertnin da oḥudu qulaqlaryča ol ulusnun (Exo 24:7)

-ind́a: anyn üčün ajtylady s. čerüvlarinin Adonajnyn ošol Vahevni Sufada (Num 21:14)

e BHeb. סָפָּר 'written document, scroll', Mod.Heb. סֶפֶר 'book', Mod.SWKar. sefer 'book'.

semiz: semiz etli 'plump (lit. of fatty meat)'

-ler: jedi tanalar körklü körümlülar da s. e. (Gen 41:2) $\Rightarrow$-lar

-lar: ol Nildan čygadylar jedi tanalar s. e. (Gen 41:18) $\Rightarrow$-ler

\& Mod.NWKar. semiz 'fatty', ModNWKar. et 'flesh, meat' + deriv. suff.

sibba postp. 'because of'

-jdan: qarg்yšly bolur ol jer senin s. (Gen 3:17); tiri bolgjaj žanym senin s. (Gen 12:13); alġyšlady meni Adonaj senin s. (Gen 30:27)

-jyzdan: ačuvlandy Adonaj siznin s. (Deu 1:37)

-syndan: bardy anar da alġyšlady Adonaj ošol üvün ol Micrilinin s. Josefnin (Gen 39:5); 
s. ošpu išnin alġyšlar seni Adonaj Tenrij (Deu 15:10); s. ol jerenči išlernin ošpularnyn Adonaj Tenrij tasetedi alarny alnyjdan (Deu 18:12)

\& Mod.NWKar. sibba '1. reason; 2. twist of fate'.

siliǵaj '1. *(body) fluid; 2. saliva'

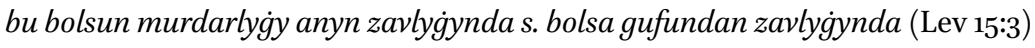

^ Mod.NWKar. śelaǵa 'saliva', Mod.EKar. slegej silegej id. In Lev 15:13, the word is perhaps used in the sense of '(body) liquid' as prompted by the context. Cf. EKar. suvan 'watery, liquid' in C (Lev 15:3).

\section{singir 'summit'}

-nin: tüzünd́a Mo’avnyn bašyna ol s. (Num 21:20); aldy any tüzüńa qaravčularnyn bašyna ol s. da qondardy jedi mizbeahlar (Num 23:14); tübüńa ol s. mizraḥ sary (Deu 3:17); mingin bašyna ol s. da kötürgün közlarijni máaravsary (Deu 3:27); tašqynlary tübüńa ol s. (Deu 4:49); mindi Moše jylġalyqlaryndan Mo’avnyn tavyna Nevonun bašyna ol s. ki alnynda Jeriḩonun (Dut 34:1)

\& Mod.SWKar. singir 'summit'.

smarak 'emerald'

-nyn: dört jergeler taštan jergesin rubinnin s. da karbankulisnin ol bir jerge (Exo 28:17);

e Russ. arch. смарагдъ 'emerald', see SRJaXI-XVII (XXV: 153). $\Rightarrow$ šmarak

sogàg 'fawn'

Naftali s. kibik ijilǵan ol berüvčü čeber ajtmaqlar (Gen 49:21); nečik ki ašaldy ošol oljür da ošol ol s. alaj ašag்yn (Deu 12:22)

-ny: ašasyn any jürnü kibik da s. kibik (Deu 12:15); s. da jürnü (Deu 14:5); jürnü kibik da s. kibik (Deu 15:22)

\& Mod.SWKar. sohaq 'fawn', Mod.NWKar. sohah id.

sö|k- 'to slander'

-|ǵajim: ne qargajym neni qarġamady Tenri da ne s. neni sök Adonaj (Num 23:8)

-kün: kelgin qarġagyn maja Ja'aqovnu da kelgin s. Jisra’elni (Num 23:7)

-ḿadi: ne qarg்ajym neni qarg்amady Tenri da ne söǵajim neni s. Adonaj (Num 23:8)

e Mod.SWKar. sek- 'to slander'.

söz 'matter; thing'

eger bar ̌̌ymaty Jisra’elnin janġylsalar dajašyryn bolsa s. ag்araqlaryndan ol qahalnyn (Lev 4:13); jaḩšy köründü közlerimizde ol s. da aldym sizden on eki erenler birer kiši ševetten (Deu 1:23); ki tamaša bolsa senden s. töre išinde arasyna qannyn qanbyla arasyna jarg்unu jarg்ubyla da arasyna ḥastalyqnyn ḥastalyqbyla talaš sözleri šaharlaryjda da turg்un barg்yn ol orunġa ki sajlasa Adonaj Tenrij any (Deu 17:8); sözüne köre eki tanyqlarnyn jemese sözüne köre üč tanyqlarnyn qajjam bolsun s. (Deu 19:15)

-leri: ki tamaša bolsa senden söz töre išinde arasyna qannyn qanbyla arasyna jarg்nu $[n]$ jarg்ubyla da arasyna hastalyqnyn hạtalyqbyla talaš s. šaharlaryjda da turgun barg்yn ol orunga ki sajlasa Adonaj Tenrij any (Deu 17:8) 
-ĺarni: qyldy Aharon da uvullary anyn ošol bar ol s. ki bujurdu Adonaj naviligi ašyra Mošenin (Lev 8:36); maġat unutursen ošol ol s. ki kördülar közlarij (Deu 4:9) -nü: töre eterediler ošol ol ulusnu harvaḥtta ošol ol qaty s. keltirirediler Mošege da bar ol kiči nerseni töre eterediler özleri (Exo 18:26); ajtty Adonaj Mošege daġyn ol s. ošpunu ki sözledij qylarmen anyn üčün ki taptyj širinlik 'enajatlarymda da bildim seni atbyla (Exo 33:17)

e Mod.NWKar. śoź 'word', cf. Heb. דָדָּ '1. word, speech; 2. matter, affair; 3. business'. stol 'table'

qylg̀yn s. šitim ag்ačlaryndan (Exo 25:23); kötürüp eltinsin alarbyla ošol ol s. (Exo 25:28); bergin ol s. üstüne eki jüzlü ötmek alnymda hammeše (Exo 25:30); qyldy ošol ol savutlarny ki ol s. üstüne (Exo 37:16); qojg்n any eki tüzüvlar altyšar hala bolsun ol bir tüzüvd́a ol aruv s. üstüna alnynda Adonajnyn (Lev 24:6); saqlavlary alarnyn ol aron da ol s. da ol čyraqpa da ol mizbeaḥlar da ol aziz savutlar (Num 3:31)

-nu: jüzükler orunlar tutqučlarġa eltme ošolols. (Exo 25:27); qojg்un ošolols. tyšqartyn parohetke da ošol ol čyraqbany uturusuna ol stolnun janynda ol miškannyn temansary da ols. bergin janynda cafonnun (Exo 26:35); ošol ol s. da ošol bar savutlaryn anyn (Exo 30:27); ošol ol s. da ošol savutlaryn anyn (Exo 31:8); ošol ol s. da ošol tutqučlaryn anyn (Exo 35:13); passim

-nun: qojg்un ošol ol stolnu tyšqartyn parohetke da ošol ol čyraqbany uturusuna ol s. janynda ol miškannyn temansary da ol stolnu bergin janynda cafonnun (Exo 26:35); qojdu ošol ol čyraqbany ohel moédde qaršysyna ol s. (Exo 40:24)

-u: s. üstüńa ol eki jüzlü ötḿaknin jajsynlar kök upraq (Num 4:7)

e Russ. стол 'table'.

stolp 'pole, pillar'

baqty qatyny anyn artyndan anyn da boldu tuzlu s. (Gen 19:26)

e Russ. arch. столпв 'pole', Russ. столб id.

stolpec *'tablecloth'

-lerin: qylg̀yn tepsilerin anyn da qašyqlaryn anyn da s. anyn da arytqyčlaryn anyn (Exo 25:29) $\Rightarrow$-ĺarin

-lerni: ol qašyqlarny ki alarbyla ölčeredi ol kohen ošol ol tütünü da ošol arytqyčlarny da ošol ol s. ki alar üstüne edi ol arytqyčlar (Exo 37:16)

-larin: arytqyčlarny da ošols. ol qaplavyn da ol hammeśalikötmák anyn üstüna bolsun (Num 4:7) $\Rightarrow$-lerin

e Russ. arch. столбецв 'a roll of fabric', столпецв 'a unit of measurement of fabric (for tablecloth)', see SRJaXI-XVII (XXVIII: 8o, 86).

stupień 'step'

-ler: minmegin s. byla anyn üstüne ki aškarmaġaj ajibij anyn üstüne (Exo 20:26)

e Russ. ступень 'step', Brus. ступень id.

sturlap 'household idol'

-larny: urlady Rahel ošol ol s. ki atasynyn (Gen 31:19); bilmedi Ja'aqov ki Raḥel urlady ol s. (Gen 31:32); tyntty Lavan da tapmady ošol ol s. (Gen 31:35) 
^ Russ. стурлабы 'gods, idols' (used in various East Slavonic translation of Genesis, see Griščenko 2018a), Mod.NWKar. sturlab 'god, idol', Mod.SWKar. sturlap id.

sukot 'booths'

alyjyz özüjüzǵa burun ol burung் künd́an s. išlaḿakka (Lev 23:40)

-ta: s. olturujuz jedi künlar bar ol jerli jisra’eld́a oltursunlar s. (Lev 23:42); snyn üčün ki bilǵajlar dorlaryjyz ki s. olturg்uzdum ošol ulanlaryn Jisra’elnin čyġarg்anym da alarny jerindán Micrinin menmen Adonaj Tenrisi alarnyn (Lev 23:43)

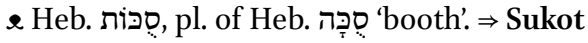

\section{Sukot 'Sukkot'}

-nun: sözlagin ulanlaryna Jisra’elnin ajtadoġač onbešinči kününd́a ol jedinči janġajnyn ošpu hyy̌̌y ol S. jedi künlar Adonajġa (Lev 23:34); hyžyn ol S. qylg̀yn özüje jedi künlük jyštyrg்anyjda yndyryjdan da čaġyrbentijden (Deu 16:13); üč keretler jylda körünsün bar erkegij qyblalary alnyna Adonaj Tenrijnin ne orunda ki sajlasa hyžynda ol macalarnyn da h̆y̌̌ynda da ḩy̌̌ynda ol Šavu'otnun da ḩy̌̌ynda ol S. da körünmesinler alnynda qyblalarynyn Adonajnyn boš (Deu 16:16); bujurdu Moše alarġa ajtadog்ač bašlyġyndan jedijyllarnyn vaġdasynda jylynyn ol šmitanyn hyž̌ynda ol S. (Deu 31:10)

e Heb. סִכּוֹ 'Sukkot'. $\Rightarrow$ sukot

sun- 'to elevate'

-du:s. any tenufa alnynda Adonajnyn (Lev 8:29); s. Aharon tenufa alnynda Adonajnyn (Lev 9:21); s. Aharon alarny tenufa alnynda Adonajnyn (Num 8:21)

-gan: qylyjyz s. künüjüzd́a (Lev 23:12)

-gun: s. alarny tenufa alnynda Adonajnyn (Exo 29:24); s. any tenufa alnynda Adonajnyn (Exo 29:26); s. alarny tenufa Adonajga (Num 8:13)

-ma: keltirsin any ošol ol töšnü s. any tenufa alnynda Adonajnyn (Lev 7:30); inčigin ol terumanyn da töšün ol tenufanyn keltirsinlar javlarybyla otlu qarbanlaryndan s. tenufa alnynda Adonajnyn (Lev 10:15); s. anyn üstüne balta (Deu 20:19)

-mady: aġalyqlaryna ulanlarynyn Jisra'elnin ki alar Nadav da Avihu’ da jetmiš kiši qartlaryndan Jisra'elnin s. naviligin berme Torany qollary ašyra alarnyn (Exo 24:11)

-mahyn: s. alar üstüne temir (Deu 27:5)

-saj: eger tašly mizbeah qylsaj alnymda qondarmaġyn any čapma tašlardan ki qylyčyjny s. anyn üstüne da jengil etersen any (Exo 20:25); s. alarny tenufa (Num 8:15)

-sun: da s. ol kohen alarny tenufa alnynda Adonajnyn (Lev 14:24); tanbylasyndan ol šabatnyn s. any ol kohen (Lev 23:11); s. ol kohen alarny (Lev 23:20); s. alarny ol kohen tenufa alnynda Adonajnyn (Num 6:20); s. Aharon ošol ol Levililarni (Num 8:11)

-uldu: aziz etkin ošol töšün ol tenufanyn da ošol inčigin ol terumanyn ki s. (Exo 29:27)

e Mod.NWKar. sun- 'to reach out'.

sus 'appearance'

-u: dajaratty Tenri ošol ol adamny s. byla s. byla malahnyn jaratty any (Gen 1:27); anyn üčün ki s. byla malahnyn jaratty Tenri [o]šol ol adamny (Gen 9:6)

-umuz: da ajtty Tenrijaratajyq adam s. [byla] (Gen 1:26) 
-una: da tiri edi Adam jüz [o]tuz jyl da tuvdurdu uqšašy byla s. köŕa (Gen 5:3)

e Mod.SWKar. sus 'appearance, shape'.

sürne: sürne tašy 'kohl stone'

ol ekinči jerge s. $t$. safir tašy da dijament (Exo 28:18); ol ekinči jerge s. t. safir tašy da dijament (Exo 39:11)

^ Mod.SWKar. sirne 'kohl, greasepaint', Mod.NWKar. śurńat id., Mod.NWKar. taš 'stone'.

sürülǵan 'divorced'

qyzy kohen\{nin\} ki bolsa tul da s. da urluq bolmasa anar da qajtsa üvüna atasynyn jašlyqlarynda kibik ötmagindán atasynyn ašasyn da heč jat ašamasyn andan (Lev 22:13)

-ni: zona qatynny da jengil bašlyny almasynlar da qatynny s. erind́an almasynlar ki azizdi ol Tenrisińa özünün (Lev 21:7); tulnu da s. erind́an da jengil bašlyny zonany ošol bularny almasyn ki ančaq boj qyzny uluslaryndan özünün alsyn qatyn (Lev 21:14)

-nin: nijeti tulnun da s. barča neki bavlady žany üstüńa qajjam bolsun anyn üstüńa (Num 30:10)

e Mod.NWKar. śur- 'to drive, to drive away'.

sürüv '1. pasture; pasture land; 2. yield'

bujurg்un ulanlaryna Jisra’elnin da bersinlar Levililarǵa ülüšünd́an tutuvluqlarynyn šaharlar olturma da s. šaharlarġa čüvŕalarind́a alarnyn (Num 35:2)

-ĺari: s. alarnyn bolsunlar tuvarlaryna da mallaryna da bar tirilarina (Num 35:3); s. ol šaharlarnyn (Num 35:4); bolsun \{alarġa\} s. ol šaharlarnyn (Num 35:5)

-ĺarin: bar ol šaharlar ki berijiz Levililarǵa qyrq segiz šahar alarny da ošol s. alarnyn (Num 35:7)

-ünün: tüzü s. šaharlarynyn satylmasyn ki ömürlük tutuvluqtur ol alarġa (Lev 25:34); da nametinden bitišlerinin qujašnyn da nametinden s. ajlarnyn (Deu 33:14)

e Mod.SWKar. siriv 'pasture', Mod.EKar. sür- 'to plough'.

syltav 'slander'

qojsa anar s. sözler da čyg்arsa anyn üstüne jaman slava (Deu 22:14); muna ol qojdu anar s. sözleri ajtadoġač tapmadym qyzyja bojluqlar da bulardylar bojluqlary qyzymnyn (Deu 22:17)

e Mod.SWKar. syltau '1. bluster; 2. slander, calumny'.

syn- 'to be injured'

-sa: ki berse kiši dostuna ešek jemese ögüz jemese qoj da nendijd́a tuvar saqlama da ölse jemese sy[ns] a jemese jesirlense bolmasa körüvčü (Exo 22:9); ki ötkünč alsa kiši dostundan da s. jemese ölse jesisi anyn bolmasa birgesine töleme tölesin (Exo 22:13)

\& Mod.SWKar. syn- 'to break, to be destroyed'.

syndan 'prison'

-ġa: da ajtty bijim Moše s. qojg̈un alarny (Num 11:28)

e Mod.NWKar. zindan zyndan 'prison'. $\Rightarrow$ syndan, zyndan üv $\sim$ zyndan üvü 
syndan: syndan üv 'prison'

-de: tung்učundan paŕonun ol olturuvču tahty üstüne tunġučunadejin ol jesirnin ki ol s. üvde (Exo 12:29)

e Mod.NWKar. zindan zyndan 'prison', Mod.NWKar. $\ddot{u}$ ' house'. $\Rightarrow$ syndan, zyndan üv zyndan üvü

synyqlyq 'fracture'

s. s. ornuna köz köz ornuna (Lev 24:20)

^ Mod.NWKar. synyhlyḥ 'humility', Mod.EKar. synyqlyq ‘. fracture; 2. weakness'.

\section{$\check{S}$}

ša'atnez 'shaatnez (cloth woven of wool and linen)'

upraq ekið̌yns š. minḿasin üstüja (Lev 19:19); kijmegin š. junnu da üskülünübirge(Deu 22:11)

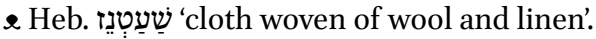

šabat 'Sabbath'

šabaton š. aziz kün Adonajġa tanbyla (Exo 16:23); saġyng்yn ošol š. künnü azizlikt́a tutma any (Exo 20:8); alg̀yšlady Adonaj ošol ol š. künnü da aziz etti any (Exo 20:11); alty künler qylynsyn iš da ol jedinči künde š. šabaton aziz Adonajġa bar ol qyluvču iš ol š. künde ölme öltürülsün (Exo 31:15); alty künlerde qylynsyn iš da ol jedinči künde bolsun sizge aziz š. šabaton Adonajga bar ol qyluvču anda iš ölme öltürülsün (Exo 35:2); jandyrmajyz ot bar olturušlaryjyzda ol š. kününde (Exo 35:3); passim

-lar: sanajyz özüjüzǵa tanbylasyndan ol šabatnyn keltirǵan künüjüzd́an ošol kültasin ol tenufanyn jedi š. tüǵalĺar bolsunlar (Lev 23:15); tüǵallar š. byla ünd́aladoġan šmitalarny ne ki tutmadylar bar bijlik künlarind́a jazylġanġa köra Torada (Lev 26:34)

-laryjyzda: bar veran bolġan künlarind́a šabat tutar ošol neki tutmady š. olturgaanyjyzda anyn üstüńa (Lev 26:35)

-larymny: ošol š. saqlajyz ki belgidir ol arama da arajyzġa dorlaryjyzsajyn bilma ki men men Adonaj aziz etüvčü sizni (Exo 31:13); ošol š. saqlajyz azizlikbyla (Lev 19:3); š. saqlajyz azizlikta (Lev 19:30); ošol š. saqlajyz da miqdašymdan qorqujuz menmen (Lev 26:2)

-laryn: sanag்yn özüja jedi š. jyllarnyn jedi jyllar jedi keŕatlar da bolurlar saja künlari jedi š. oljyllarnyn qyrq tog்uzjyl (Lev 25:8); ol vahtta tüǵallar ol jer ošol š. özünün (Lev 26:34); da tüǵallar ošol š. (Lev 26:43)

-larynyn: sanag்yn özüja jedi šabatlaryn jyllarnyn jedi jyllar jedi kératĺar da bolurlar saja künlarijedi š. ol jyllarnyn qyrq tog்uzjyl (Lev 25:8)

-laryndan: bašqa š. Adonajnyn (Lev 23:38)

-ny: Adonaj berdi sizge ol š. (Exo 16:29); saqlajyz ošol ol š. ki azizdi ol sizge (Exo 31:14); da saqlasynlar ulanlary Jisra’elnin ošol ol š. azizlikte tutma ošol ol š. dorlarysajyn ömürlük šert (Exo 31:16) 
-nyn: sunsun ošol ol kült́ani alnynda Adonajnyn qabulluqqa siznin üčün tanbylasyndan ol š. sunsun any ol kohen (Lev 23:11); tanbylasynadejin ol jedinči š. sanajyz enli kün da juvutujuz janġy tirki Adonajg̉a (Lev 23:16); sanajyz özüjüzǵa tanbylasyndan ol š. keltirǵan künüjüzd́an ošol kült́asin ol tenufanyn jedi šabatlar tüǵallar bolsunlar (Lev 23:15); 'olasyn har š. šabatynda olasy qatyna ol hammeśalik qarbannyn da nesehi anyn (Num 28:10)

-ty: ašajyz any bügün ki š. bügün (Exo 16:25); alty künler čöplejiz any da ol jedinči künde š. bolmasty anda (Exo 16:26); ol jedinči kün š. (Exo 20:10); heč iš qylmajyz š. ol Adonajgia (Lev 23:3); ol jedinči kün š. mahtavuna Adonaj Tenrijnin qylmaġyn heč iš (Deu 5:14)

-y: bolsun š. ol jernin sizǵa (Lev 25:6)

-yjyzny: ingird́an ingirǵadejin šabat tutujuz ošol š. (Lev 23:32)

-ynda: 'olasyn har šabatnyn š. olasy qatyna ol hammeśalik qarbannyn da nesehi anyn (Num 28:10)

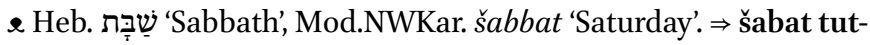

šabat tut- 'to rest; to keep a Sabbath'

-ar: da siz bolursiz jerind́a dušmanlaryjyznyn ol vahtta veŕanliksartyn š. t. ol jer da tüǵallar šabatlar byla ünd́aladoġan šmitalarny ne ki tutmadylar bar bijlik künlarind́a jazylġanġa köŕa Torada (Lev 26:34); bar veŕan bolġan künlarind́a š. t. ošol neki tutmady šabatlaryjyzda olturg்anyjyzda anyn üstüńa (Lev 26:35)

-qun: alty künlerd́a išlegin išlerijni da ol jedinči künde š. t. anyn üčün ki tynč algiaj ögüzüj da ešegï da tynč algaj uvlu qaravašyjnyn da ol garipde (Exo 23:12); alty künlerde išlegin išlerijni da ol jedinči künde š. t. saban sürmek zamanda da ormaq zamanda š. t. (Exo 34:21)

-sun: sözlagin ulanlaryna Jisra’elnin da ajtqyn alarġa ki kelśajiz ol jerǵa ki men beŕamen any sizǵa da š. t. ol jer šabat Adonajg̉a (Lev 25:2)

-tular: š. t. ol ulus ol jedinči künde (Exo 16:30)

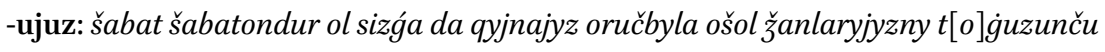
kününd́a janġajnyn ingird́a ingird́an ingirǵadejin š. t. ošol šabatyjyzny (Lev 23:32)

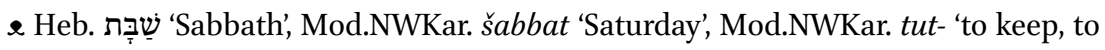
hold'. $\Rightarrow$ šabat

šabaton 'the day of complete rest; complete rest'

ajtty alarġa oldu ki sözledi Adonaj š. šabat aziz kün Adonajg̉a tanbyla (Exo 16:23); alty künler qylynsyn iš da ol jedinči künde šabat š. aziz Adonajg̉a (Exo 31:15); ol jedinči künde bolsun sizge aziz šabat š. Adonajga (Exo 35:2); tek onbešinči kününd́a ol jedinči janġajnynjyštyrg்anyjyzda ošol bitišin ol jernin hyžlajyz ošol hyžyn Adonajnynjedi künlar ol burunġu künd́a š. da ol segizinči künd́a š. (Lev 23:39); ol jedinči jylda šabat š. bolsunjerǵa šabat Adonajġa (Lev 25:4); passim

-dur: šabat š. ol sizǵa da qyjnajyz oš ol క̌anlaryjyzny ömürlük resim (Lev 16:31)

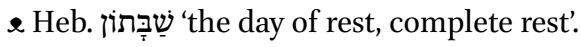




\section{šaḥaf 'seagull'}

-ny: ošol ol anqyt balasyn da ošol ol tạ̣masny da ošol ol š. da ošol ol qyrg்yjny žynsysajyn (Lev 11:16); ošol ol anqyt balasyn da ošol tạ̣masny da ošol š. da ošol ol qyrg்yjny з̌ynsysajyn (Deu 14:15)

\& Heb. שֵָׁ 'seagull'.

\section{šajtan 'devil'}

-larġa: debeḥa etḿasinlar artyq ošol debehalaryn özlarinin š. ki alar azadylar alar artyna ömürlük resim bolsun bu alarġa dorlarysajyn (Deu 32:17)

e Ar. شيطان 'devil', Mod.NWKar. šyjtan 'Satan, devil', Mod.SWKar. sejtan id. $\Rightarrow$ šejtan šalah *'cormorant'

-ny: ošol ol kosnu da ošol ol š. da ošol ol janšufnu (Lev 11:17)

\& Heb. שִָָׁ 'a bird of uncertain meaning, probably the cormorant', see Klein (1987: 662), 'unclean bird; cormorant (?) or fish-owl (?)', see Koehler \& Baumgartner (1985: 978).

šalom ber- 'to offer peace, to say goodbye'

-di: š. b. Ja'aqov paróga da čyqty alnyndan par'onun (Gen 47:10)

e Mod.SWKar. šalom ber- 'to greet, to send greetings', see Németh (2011b: 318).

\section{šatan 'adversary'}

ajtty anar malahy Adonajnyn ne üčün urduj ošol eśagïni bu üč qurlalar muna men čyqtym š. bolmaqqa ki qyng்yr köründü ol jol alnymda (Num 22:32)

-ga: qabundu ačuvu Adonajnyn ki bardy ol da turdu malahy Adonajnyn jolda š. anar (Num 22:22)

\& Heb. שָָָׁ 'adversary'.

\section{šavağat 'kindness'}

haligińa antetkin maja Tenrid́an bunda eger aldasaj maja da unug்uma da praunuguma š. kibik ki qyldym birǵaja qylg̀nn birgeḿa (Gen 21:23); da qylġyn š. bijim Avraham byla (Gen 24:12); da anlyq byla bilirmen ki qyldyj š. bijim byla (Gen 24:14); qylamosiz š. da kertilik bijimbyla anlatyjyz maja (Gen 24:49); da edi bolušluġu Adonajnyn Josef byla da sundu anar š. da berdi ošol širinligin közlerind́a ag்arag்ynyn ol zyndan üvünün (Gen 39:21); qylg்yn endi birgeḿa š. da sag்yndyrg்yn meni par'og்a (Gen 40:14); qyluvčudur men š. min dorlarġa (Exo 20:6); qyluvčudur men š. min dorlarg்a (Deu 5:10)

-lardan: kiči boldum bar ol š. da bar ol kertilikten ki qyldyj quluj byla (Gen 32:11)

-ny: ol inamly Tenri saqlavču ol šertni da ol š. süvüvčülarǵa oḥuma Torasyn (Deu 7:9); da saqlar Adonaj Tenrij saja ošol ol šertin da ošol ol š. ki antetti atalaryja (Deu 7:12) -y: bu bolsun š. ki qylg̀yn birgeḿa bar ol orunġa ki kelsek ary ajtqyn menim üčün qaryndašymdy ol (Gen 20:13)

-yj: köndergin š. byla bu ulusnu (Exo 15:13)

-yjny: muna endi tapty quluj širinlik közlerijd́a da ulgiajtyj š. ki qyldyj birǵaḿa tiri qaldyrma ošol žanymny (Gen 19:19) 
-yjnyn: bošatqyn endi qoltqabyla günahịn ol ulusnun ošpunun ulluluġunaköra š. (Num 14:19)

-yn: maḥtavludu Adonaj Tenrisi bijimnin Avrahamnyn ki kemišmedi š. (Gen 24:27)

\& Mod.SWKar. savahat 'kindness, mercy, favour'. $\Rightarrow$ šavagatsyz

šavaġatsyz 'merciless'

uvu aždahalarnyn čaġyrlary alar [nyn] da uvu š. aždahalarnyn (Deu 32:33)

e Mod.SWKar. savahatsyz 'merciless'. $\Rightarrow$ šavaġat

Šavu'ot 'Shavuot'

-nun: hyšyn Š. qylġyn özüje jetilmeklerin ormaqlarynyn budajlarnyn da hyžyn oljyštyrmaqnyn ajlanmaġynda ol jylnyn (Exo 34:22); qylg̀yn özüje hyžyn Š. alnynda Adonaj Tenrijnin (Deu 16:10); hyšynda ol Š. da hyy̌ynda ol Sukotnun (Deu 16:16)

e Heb. שָבוּעוֹת 'Shavuot, the Feast of the Weeks'.

šefela 'lowland'

-da: kelijiz tavyna ol Emorinin da bar qonšularyna anyn jylġalyqta tavda da š. da tüšlükte (Deu 1:7)

^ Heb. نשָפָָ 'lowland'.

šehina 'divine Presence (of God)'

qorqtu baqmaqtan ol š. üstüne (Exo 3:6)

-j: ašmasyn š. quluj qatyndan (Gen 18:3)

-jnyn: qajjam š. toḥtamaġyna (Exo 15:17)

-m: š. turdy alnyjda (Exo 17:6); muna kelir š. menim saja bazyq bulut byla (Exo 19:9); tohtar š. ortalarynda alarnyn (Exo 25:8); aškara bolma š. saja (Exo 25:22); ki hadir bolur š. aškara bolma sizge (Exo 29:42); passim

-mnyn: ašqanynadejin š. (Exo 33:22); berirmen toḥtar ornun š. ortajyzda (Lev 26:11) -nyn: balquvu š. (Deu 33:2)

-sy: š. Adonajnyn (Gen 4:4); da toḥtagaj š. Tenrinin (Gen 9:27); e[ndi] š. Adonajnyn baqma (Gen 11:5); aškara boldu š. Adonajnyn (Gen 12:7; 17:1); da sözledi š. Tenrinin (Gen 17:3); passim

-syn: kördüler ošol š. Tenrisinin Jisra’elnin (Exo 24:10); kördüler ošol š. ol Tenrinin (Exo 24:11); da š. Adonajnyn bagady (Num 12:8)

-syna: budur Tenrim da orun hadirlejim š. (Exo 15:2)

-synyn: körümü š. Adonajnyn (Exo 24:17)

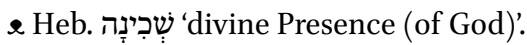

šejtan 'devil'

-larg்a: debeḥa etüvčü š. ḥeremli bolsun ančaq Adonajga jalġyż̇a (Exo 22:19); debeḥa ettiler š. tüvül Tenrige abaqlarġa (Deu 32:17)

ع Ar. شيطان ‘devil', Mod.NWKar. šyjtan 'Satan, devil', Mod.SWKar. sejtan id. $\Rightarrow$ šajtan šelamim '( $p l$. t.) peace offering'

ijdi ošol tung்uč ulanlaryn uvullarynyn Jisra'elnin da čyg்ardylar 'olalar da debeha ettiler š. debeḥalary alnynda Adonajnyn tanalar (Exo 24:5); ašadylar š. debehalardan 
da ičtiler (Exo 24:11); juvutsun ol š. debehasyndan otlu qarban Adonajg̈a (Lev 3:3); ol qojdan bolsa qarbany anyn olš. debeḥasyna Adonajga (Lev 3:6); juvutsun olš. debeḥasyndan otlu qarban Adonajga (Lev 3:9); nečik ajyrylady ögüzünd́an ol š. debehasynyn da tütatsin alarny ol kohen mizbeahy üstüńa ol olanyn (Lev 4:10); ošol barjavyn anyn tütatsin ol mizbeahta jarynkibik ol š. debehasynyn (Lev 4:26); passim

-ǵa: ögüz da qočqar š. debeḥa etḿa alnynda Adonajnyn (Lev 9:4); juvutsun ošol qarbanyn özünün Adonajg்a [...] qočqar tüǵalni š. (Num 6:14)

-ler: tünlej turdular tanbyladan da čygardylar 'olalar da juvuttular š. (Exo 32:6)

-lerijni: mizbeaḥjerden qylg̀yn alnymda da debeḥa etkin anyn qatyna ošol ólalaryjny da ošol š. (Exo 20:24)

-lerinin: teruma bolsun ulanlaryndan Jisra'elnin debehalaryndan š. terumalary alarnyn Adonajga (Exo 29:28) $\Rightarrow$-ĺarinin

-ĺarijiz: bulardylar ol musaflary ki qylyjyz Adonajġa mo'edĺarijizd́a bašqa nijetlarijizd́an da žomartlyqlaryjyzdan olalaryjyzsajyn da tirkilarijizsajyn da nesehllarijizsajyn da š. sajyn (Num 29:39)

-larijiznin: tartyjyz byrg்ylar byla olalaryjyz qatyna da debehasy qatyna š. da bolsunlar sizǵa saġynčlyqqa (Num 10:10)

-ĺarinin: halalarybyla qužur ötḿaknin juvutsun qarbanyn özünün debehasy qatyna šükürlük š. (Lev 7:13); eger ašalma ašalsa etind́an debehasynyn ol š. ol üčünčü künd́a (Lev 7:18); keltirsin ošol qarbanyn özünün [A]donajga debehasyndan š. (Lev 7:29); ošol inčigin ol terumanyn aldym ulanlaryndan Jisra'elnin debehalaryndan š. da berdim alarny Aharonġa ol kohenǵa (Lev 7:34) $\Rightarrow$-lerinin

-ĺarnin: eti debehasynyn šükürlük š. juvutqun (Lev 7:15); ol juvutuvču ošol qanyn ol š. (Lev 7:33)

-ni: alġyšlady alarny andan sortun nečik endi qylmaqtan ol hatatny da ol 'olany da ol š. (Lev 9:22)

-nin: eger debehasy š. bolsa qarbany anyn (Lev 3:1); ošol bar javyn anyn ketarsin ki nečik ketariladi jav debehasy üstünd́an ol š. (Lev 4:31); tütatsin anyn üstüna javlaryn ol š. (Lev 6:5); juvutsun andan birni bar qarbandan teruma Adonajga kohenǵa ol bürküvčüǵa ošol qanyn ol š. anar bolsun (Lev 7:14); budur ol üvŕatüv olaġa da tirkiǵa da ḥatatqa da ašamġa da milu’imǵa da debeḥasyna ol š. (Lev 7:37); ol debehasyny š. eki syġyr (Num 7:65); qoj da syg்yr debehasyna š. (Deu 16:2)

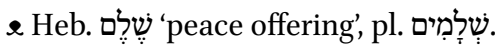

ševo 'agate'

ol üčünčü jerge lešem š. da aḥlama (Exo 28:19); ol üčünčü jerge lešem š. da aḥlama (Exo 39:12)

\& Heb. שְׁב 'name of a precious stone, probably identical with the agate'.

šipšin 'prickle'

-larǵa: eger tasetmááajiz ošol olturuvčularyn ol jernin alnyjyzdan da bolgaj ki qaldyrsajyz alardan bolurlar š. közlarïizd́a (Num 33:55) 
^ Mod.SWKar. sipsinja 'prickle, thorn', cf. Pol. szypszyna 'a rose species with stems covered with prickles', Ukr. шипшина id.

\section{šitim 'acacia'}

terilerin tahašlarnyn da š. ag்ačlaryn (Exo 25:5); qylsynlar ošol ol aronnu š. ag்ačlaryndan (Exo 25:10); qylg̀yn tutqučlar š. aġačlaryndan (Exo 25:13); qylg̀yn stolš. ag்ačlaryndan (Exo 25:23); qylġyn ošol ol tutqučlarny š. aġačlaryndan (Exo 25:28); passim

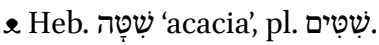

šmarak 'emerald'

-nyn: jergesi rubinnin š. da karbankulisnin (Exo 39:10)

e Pol. szmaragd 'emerald'. $\Rightarrow$ smarak

šmita 'remission'

qystamasyn ošol dostun da ošol qaryndašyn ki čag்yrdy š. Adonajg̉a (Deu 15:2)

-lar: ol vahtta tüǵallar ol jer ošol šabatlaryn özünün ki oldur ol š. (Lev 26:34)

-larny: ol vahtta veŕanliksartyn šabat tutar ol jer da tüǵallar šabatlar byla ündáladogan š. (Lev 26:34)

-nyn: budur iši ol š. (Deu 15:2); juvudu ol jedinči jyl jyly ol š. (Deu 15:9); jedi jyllarnyn vaǵdasynda jylynyn ol š. (Deu 31:10)

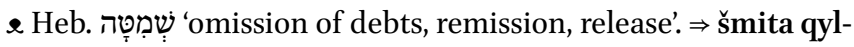

šmita qyl- '1. to let lie fallow; 2. to make a remission'

-̇̀yn: ol jedinči jylda š. q. anar (Exo 23:11); aḥerinden jedijyllarnyn q. š. (Deu 15:1)

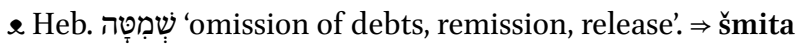

šofar 'trumpet'

tartqanda ol š. išlengen qočqar müvüzünd́an olvahtta barsynlar čatyrlaryndan turma ol tav tübüne (Exo 19:13); boldu avazlar da jyldyrymlar da küčlü bulut ol tav üstüne da š. avazy küčlü astry (Exo 19:16); edi ol š. avazy baryredi da küčejiredi astry Moše sözleredi da ol Tenri arttyryredi anar küč avazynda ki ešitkejler ulanlary Jisra’elnin sözlerin Mošenin tartmaq vahtta ol š. (Exo 19:19); bar ol ulus körerediler ošol ol avazlarny da ošol ol küsövlerni da ošol ol š. avazyn (Exo 20:18); ašyrg்yn qyčqyrmaqbyla š. avazy ol jedinči janġajda onunču kününd́a janġajnyn ol Kipurim kününd́a ašyryjyz š. avazy bar jerijizd́a (Lev 25:9)

e Heb. שiֹ 'trumpet'.

šoham 'onyx'

andady ol inži tübü [da ol] š. tašy (Gen 2:12); š. tašlary efodg்a (Exo 25:7); alġyn ošol ol eki š. tašlarny (Exo 28:9); ol dörtünčü jerge taršiš tašy da š. (Exo 28:20); š. tašlary efodġa (Exo 35:9); keltirdiler ošol ol š. tašlaryn efodġa (Exo 35:27); qyldylar ošol ol š. tašlaryn (Exo 39:6); ol dörtünčü jerge taršiš š. da jašfe quršalganlar altyn ojuvlarbyla tolturmaqlarynda (Exo 39:13)

e Heb. שنהם 'name of a precious stone, generally identified wth the onyx'.

šorba 'juice'

-syn: bar š. borlalarnyn ičḿasin (Num 6:3)

e Mod.NWKar. šorba 'soup'. 
tag

-qanda: kelḿasinlar baqma t. ošol ol qodešni da ölarĺar (Num 4:20)

\& Cf. Kirg. and Tar. taqa- '1. to bolt; to barricade; 2. to cover, to close', Tar. takat- 'to bolt; to barricade', see Radlov (1905: III/1: 780, s.v. maka-). The verb jap- 'to cover' is used instead of it in the alternative translation of the verse Num 4:20 provided by the copyist (see $215 \mathrm{v}^{\mathrm{o}}$ ).

taḥaš *'porpoise'

bersinlar anyn üstüna qaplavyn t. terisinin (Num 4:6); qaplasynlar any qaplav byla t. terisind́an (Num 4:8); qaplasynlar any qaplav byla t. terisind́an (Num 4:11); qaplasynlar alarny qaplav byla t. terisind́an (Num 4:12); da jajsynlar anyn üstüna qaplav $t$. terisind́an da qojsunlar tutqučlaryn anyn (Num 4:14); passim

-larnyn: terilerin qočqarlarnyn qyzartylġanlarny da terilerin $t$. (Exo 25:5); terilerinden t. (Exo 26:14); da terilerin t. (Exo 35:7); da terilerin t. keltirdiler (Exo 35:23); da qaplav terilerinden $t$. (Exo 36:19); passim

\& Heb. תָחָש 'porpoise', see Koehler \& Baumgartner (1985: 1026). Klein (1987:699), in turn, treats this word as of uncertain etymology and meaning and translates it as 'dolphin (?)'

taḥmas 'nighthawk (?)'

-ny: ošol ol anqyt balasyn da ošol ol t. da ošol ol šaḥafny da ošol ol qyrg்yjny žynsysajyn (Lev 11:16); ošol ol anqyt balasyn da ošol t. da ošol ša ḥafny da ošol ol qyrg்yjny žynsysajyn (Deu 14:15)

\& BHeb. תַחְָמָס 'an unclean bird of prey', see Klein (1987:698), 'an unclean bird of prey, perhaps a species of owl', see Koehler \& Baumgartner (1985: 1025). It is interpreted as 'nighthawk' in KJ200o, ESV, and by Friedman (2003).

tame 'unclean'

t. t. čag்yrsyn (Lev 13:45)

\& Heb. טָמָא 'unclean, defiled'.

tan- 'to rebel'

-dyjyz: jyštyrylsyn Aharon uluslaryna ki kelḿasti ol jerǵa ki berdim ulanlaryna Jisra’elnin anyn üčün ki t. bujruġuma išind́a talaš suvlarynyn (Num 20:24); ki nečik t. maja ki qylmadyjyz sözümnü midbarynda Cinnin (Num 27:14); da klamádijiz barma da $t$. bujruġuna Adonajnyn Tenrijiznin (Deu 1:26); da sözladim sizǵa da tynlamadyjyz da t. bujrug்una Adonaj Tenrijiznin (Deu 1:43); t. bujrug்una Adonajnyn Tenrijiznin da inanmadyjyz anar da tynlamadyjyz ünüńa anyn (Deu 9:23)

e Mod.NWKar. tan- '1. to contradict, to deny; 2. to betray', Mod.EKar. tan- '1. to contradict, to deny; 2. to rebel; 3 . to betray'. $\Rightarrow$ tanuvču

tana 'cow'

-lar: muna ol Nild́an čyǵadylarjedit. körklü körümlülar (Gen 41:2); munajedit. özgelar čýgadylar alar artyna ol Nild́an (Gen 41:3); ašadylar ol t. körklü ol körümlülerni da ol 
semizlerni (Gen 41:4); muna ol Nildan čyǵadylar jedi t. semiz etlilar (Gen 41:18); jedi t. özgelar čyǵadylar alar artyna (Gen 41:19); ašadylar ol t. ol uvaqlar da ol jamanlar ošol jedi ol tanalarny ol burungularny ol semizlerni (Gen 41:20); jedi t. ol jahšylar jedi jyllardylar alar (Gen 41:26); jedi ol t. ol uvaqlar da ol jamanlar ol čyg்uvčular alar artyna jedijyllardylar alar (Gen 41:27)

-larny: ašadylar ol tanalar ol uvaqlar da ol jamanlar ošol jedi ol t. ol burung்ularny ol semizlerni (Gen 41:20)

-larnyn: boš etliler da turdular janynda ol t. qyryj üstüna ol Nilnin (Gen 41:3)

\& Mod.NWKar. tana 'heifer; calf'.

tanbylagiy 'tomorrow's'

da tanyqlyq čygar rastlyg்ym menim üčün t. künd́a (Gen 3o:33); turdu bar ol ulus bar ol künnü da bar ol kećani da bar ol t. da künnü (Num 11:32)

e Mod.NWKar. tandahy 'tomorrow's'.

tanuvču '1. rebel; 2. rebelious'

qajtargyn ošol tajaġyn Aharonnun alnyna ol šarajatnyn saqlavġa t. el üčün (Num 17:25); bolsa kišige uvul azuvču da t. tynlamavču sözüne atasynyn da sözüne anasynyn (Deu 21:18)

-du: ajtsynlar qartlaryna šaharynyn uvlumuz bu azuvču da t. tynlamajdy sözümüzge (Deu 21:20)

-lar: jyštyrdy Moše da Aharon ošol ol qahalny alnynda ol skalanyn da ajtty alarġa tynlajyz endi e t. ošpumo skaladan čyǵarajyq sizǵa suv (Num 20:10); saġyngyn unutmag்yn ošol neki ačyrg்andyrdyj Adonaj Tenrijni midbarda ol künd́an ki čyqtyjjerind́an Micrinin kelǵanijizǵadejin ošpu orunġadejin t. edijiz sözüna Adonajnyn (Deu 9:7); $t$. edijiz alnynda Adonajnyn bilǵan künümáan sizni (Deu 9:24); bügün t. edijiz alnynda Adonajnyn (Deu 31:27)

2 Mod.NWKar. tan- '1. to contradict, to deny; 2. to betray', Mod.EKar. tan- '1. to contradict, to deny; 2 . to rebel; 3 . to betray'. $\Rightarrow$ tan-

tap- 'to discover'

-ty: Lemehnnin ki ol t. ustasyn temirnin dunjada (Gen 4:23); Lemeh üčün ki ančaq t. ol čerüv savutlarny (Gen 4:24)

e Mod.NWKar. tap- 'to find'.

taršiš *'beryl'

ol dörtünčü jerge t. tašy da šoham da jašfe (Exo 28:20); ol dörtünčü jerge t. šoham da jašfe (Exo 39:13)

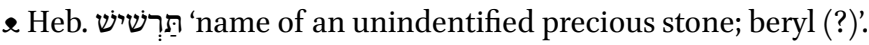

tavusadogan 'terminal (of a disease)'

cara'at t. ol hastalyq murdardy ol (Lev 13:51); t. cara'atty ol (Lev 13:53); kelsin ol kohen da baqsyn da muna jajyldy ol hastalyq üvd́a t. cara'atty ol üvd́a murdar bolsun ol (Lev 14:44)

e Mod.NWKar. tavus- '1. to destroy; 2. to finish'. 
telirmek 'madness'

urar seni Adonaj t. byla (Dut 28:28)

e Mod.SWKar. telirmek 'madness'.

temarim 'palm trees'

-nin: enišin Jeriḥonun šaharyn ol t. (Deu 34:3)

\& Heb. תִמָרָר 'palm tree'.

tenufa 'wave offering'

da sungun alarny t. alnynda Adonajnyn (Exo 29:24); da sung்un any t. alnynda Adonajnyn (Exo 29:26); sunma any t. alnynda Adonajnyn (Lev 7:30); da sundu alarny $t$. alnynda Adonajnyn (Lev 8:27); da sundu any t. alnynda (Lev 8:29); passim

-g்a: eger jarly bolsa ol arynuvču da jetmáśa küčü qolunun da alsyn bir qozu qarbanyn ašamnyn t. bošatlyq qolma özü üčün (Lev 14:21)

-lary: budu saja terumasy berńalarinin bart. sajyn ulanlarynyn Jisra’elnin (Num 18:11) -nyn: aziz etkin ošol töšün ol $t$. (Exo 29:27); da edi altyny ol $t$. (Exo 38:24); tuču ol $t$. jetmiš qantar (Exo 38:29); ošol töšün ol t. (Lev 7:34); ošol töšün ol t. (Lev 10:14); passim

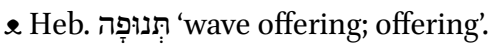

tepeten 'on one level, in one line, evenly'

ediler tenler ašaġartyn da birge ediler t. bašyna anyn ol bir jüzükke alaj qyldy eksilerinde eki ol müvüšlerge (Exo 36:29)

^ Tksh. dial. teppeten 'on one level', see Ds (x: 3885$). \Rightarrow$ teppeten

teppeten 'on one level, in one line, evenly'

bolsunlar tenler ašaġartyn da birge bolsunlar t. bašyna anyn ol bir jüzükke alaj bolsun eksilerinede eki ol müvüšlerge bolsunlar (Exo 26:24)

e Tksh. dial. teppeten 'on one level', see Ds (x: 3885$)$. $\Rightarrow$ tepeten

tereč́a 'window'

da baqty Avimeleh biji Pelištimnin ol t. ašyra da kördü (Gen 26:8)

e Mod.NWKar. teréź $\sim$ terž́a teraźa 'window'.

ters 'defiant'

bu boldu ajipleri alarnyn anyn üčün ki alar dor t. da tornaqšy (Deu 32:5)

e Mod.NWKar. terś 'opposite, reverse', Mod.SWKar. ters ‘1. opposite, reverse; 2. defiant, disobedient'.

teruma 'heave offering, terumah; contribution form produce to be set apart for priests' da bersinler maja t. har kišiden ki žomartlatsa any jüregi anyn alyjyz ošol terumamny (Exo 25:2); da budu ol t. ki alyjyz alardan altyn da kümüš da tuč (Exo 25:3); t. Adonajg்a (Exo 30:13); alyjyz özüjüzden t. Adonajga (Exo 35:5); juvutsun andan birni bar qarbandan t. Adonajgja kohenǵa (Lev 7:14); passim

-dyr: bolsun Aharonġa da uvullaryna anyn ömürlük resimge ulanlaryndan Jisra’elnin ki t. ol da teruma bolsun ulanlaryndan Jisra'elnin debehalaryndan šelamimlerinin terumalary alarnyn Adonajġa (Exo 29:28)

-jyz: saġy̌lanyr sizǵa t. (Num 18:27) 
-lary: teruma bolsun ulanlaryndan Jisra’elnin debehalaryndan šelamimlerinin t. alarnyn Adonajga (Exo 29:28)

-laryn: bar t. ol qadašimnin ki ajyrsalar ulanlary Jisra’elnin Adonajgja (Num 18:19) -mny: da bersinler maja teruma har kišiden ki žomartlatsa any jüregi anyn alyjyz ošol t. (Exo 25:2) -ny: aldylar alnyndan Mošenin ošol bar ol t. (Exo 36:3) -nyn: aziz etkin ošol töšün ol tenufanyn da ošol inčigin ol t . (Exo 29:27); ošol töšün ol tenufanyn da ošol inčigin ol t. aldym ulanlaryndan Jisra’elnin debehalaryndan šelamimlarinin da berdim alarny Aharonġa ol kohenǵa (Lev 7:34); inčigin ol t. da töšün ol tenufanyn keltirsinlar (Lev 7:15); inčigi byla ol $t$. (Num 6:20); edi bar altyny ol $t$. ki ajyrdylar Adonajga on alty min jedi jüz da enli mitqal (Num 31:52); passim

-sy: ilkin hamurlaryjyznyn hala ajyryjz teruma t. kibik (Num 15:20); budu saja t. bernalar\{i\}nin bar tenufalarysajyn ulanlarynyn Jisra'elnin (Num 18:11)

-syn: bürtügüjnün da čaġyrbentijnin $t$. berme kečiktirmegin tunġučun ulanlaryjnyn bergin maja (Exo 22:28); bar ol ašuvču ol sanalġanlar qatyna egirmi jaštan da jog்arraq bersin ošol t. Adonajnyn (Exo 30:14); bar žomart jürekli keltirsin ošol t. Adonajnyn altyn da kümüš da tuč (Exo 35:5); keltirdiler ošol t. Adonajnyn išine ohel mo'ednin (Exo 35:21); bar ajyruvču t. kümüšnün da tučnun keltirdiler ošol t. Adonajnyn (Exo 35:24); passim

-syna: kiši da qatyn qylmasynlar artyq iš t. ol qodešnin da qaldy ol ulus keltirmekten (Exo 36:6)

-syndan: qyzy kohennin bolsa jat kiši artyna ol t. ol qadašimnin ašamasyn (Lev 22:12)

-synyn: berdi Moše ošol tymġasyn t. Adonajnyn El'azarg்a ol kohenǵa (Num 31:41)

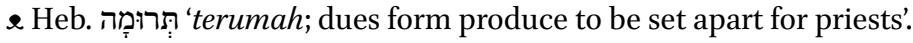

tinšamet 'chameleon'

ol anaqa da ol kovah da ol leta'a da ol homet da ol t. (Lev 11:30)

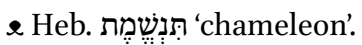

tigi *'alone, himself alone'

ajtty ol qulgia kimdi ol kiši ol t. ol jürüvčü tüzd́a uturumuzg்a (Gen 24:65); ajttylar kiši qaryndašyna muna jesisi ol tüšlernin ol t. keldi (Gen 37:19)

e An obscure form. Perhaps a 3 rd pers. sg. poss. - $i$ form of Kar. tek 'only'; the $-i$ - in the initial syllable might either be a result of an $e^{-}>-i$ - assimilation or it could be a closed $[\dot{\mathrm{e}}]$ rendered with the letter yodh. Used only in the Book of Genesis.

tigimla- 'to break into pieces'

-ḿa: $t$. any tigimlar da qujg்un any üstüńa jav tirkidi ol (Lev 2:6)

e Mod.SWKar. tigimle- 'to break into pieces'.

tinšemet 'white owl'

-ni: ošol ol t. da ošol ol qa’atny da ošol ol rahamny (Lev 11:18); ošol ol kosnu da ošol ol janšufnu da ol $t$. (Deu 14:16) 


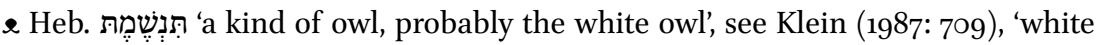
owl', see Koehler \& Baumgartner (1985: 1035).

tiri: tiri žan 'living creature, living being; animal'

jaratty Adonaj Tenri ol jerd́an bar kijigin ... da keltirdi ol Adamġa ... da bar t. ̌̌. ki atasajedi anar ol Adam at ol edi aty anyn (Gen 2:19); bar ol $t$. ̌̆. byla ki birgejizǵa (Gen 9:10)

-dan: bar ol t. ̌̆. ki suvlarda qusqunčudu alar sizǵa (Lev 11:10)

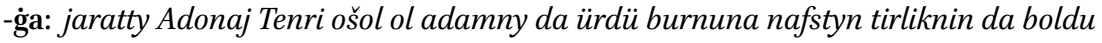
ol [adam qajjam] t. ̌̆. (Gen 2:7)

-ny: jaratty Tenri ... ošol ol bart. ̌̌. (Gen 9:21);

-nyn: qozlasynlar ol suvlar qozlamag்nn $t$. ̌̆. (Gen 1:20); arajyzǵa da arasyna bar $t . \check{3}$. (Gen 9:12); šertimni ki arama da arajyzg்a da arasyna bar $t$. ̌̆. (Gen 9:15)

e Mod.NWKar. tiri 'living, alive', Mod.NWKar. žan '1. soul; 2. life'.

tiri: tiri suvlar '1. spring water, springing water; 2 . running water'

mančsyn ... ošol ol tiri qušnu qanyna ol sojulġan qušnun ol t. s. üstüna (Lev 14:6); sojsun ošol ol bir qušnu čerep savut üstüna t. s. üstüna (Lev 14:50); da bürksün ošol ol üvnü qanybyla ol qušnun da ol t. s. byla (Lev 14:52); da juvsun upraqlaryn da juvsun gufun t. s. byla da arynyr (Lev 15:13)

-nyn: taptylar anda qujusun t. s. (Gen 26:19)

e Mod.NWKar. tiri 'living, alive', Mod.NWKar. suv 'water'.

tirilmüš '1. inhabited; 2. living, sojourning'

berirmen saja da urlug்uja özüjd́an sortun ošol t. jerlerijni ošol bar jerin Kenáannyn (Gen 17:8); bergej saja ošol alġyšyn Avrahamnyn saja da urlug்uja birgeja meresleḿa sen ošol t. jerijni ki berdi Tenri Avrahamġa (Gen 28:4); jamanlar edilar künleri tirlik jyllarymnyn da jetelmedilar ošol künlerin tirlik jyllarynyn atalarymnyn t. künlerind́a (Gen 47:9); Šimon da Levi qaryndašlar avanlyq savutlar $t$. orunlarynda (Gen 49:5); qajjam ettim ošol šertimni birgelerine berḿa alarġa ošol jerin Kena'annyn ošol t.jerlerin ki tirilediler anda (Exo 6:4)

-lerinin: edi mallary alarnyn köbüsü olturmaqtan birǵa da bolalmady jeri t. kötürḿa alarny (Gen 36:7); olturdu Ja'aqov jerind́a t. atasynyn jerind́a Kena'annyn (Gen 37:1)

e Mod.NWKar. tirilmiš 'inhabited'.

tirjak 'balm'

da teveleri alarnyn eltirediler otjamlar da t. da mastik (Gen 37:25); endirijiz kišiǵa tirki azg்ynaq t. da azg்ynaq čyjbal otjamlar da mastik zymzymlar da badamlar (Gen 43:11)

e Mod.NWKar. tirjak 'stimulant', cf. BHeb. צָּרִ 'balm' in Gen 37:25 and Gen 43:11.

tirki 'grain offering'

క̌an ki juvutsa qarban t. Adonajġa öźak bolsun qarbany anyn da qujsun anyn üstüńa jav da bersin anyn üstüńa levona (Lev 2:1); ki juvutsaj qarban t. biširilǵan pečta öźak halalar macalarjumurulganlarjavbyla dajuġa macalar jag்lganlarjavbyla (Lev 2:4); 
eger t. išlanǵan ol tava üstüńa bolsa qarbanyj öźak jumurulgan javbyla maca bolsun (Lev 2:5); eger t. išlanǵan panv́ada bolsa qarbanyj öźakjavbyla qylynsyn (Lev 2:7); bar ol t. ki juvutsajyz Adonajga qylynmasyn hamec (Lev 2:11); passim

-di: tigimláma any tigimlar da qujg்un any üstüńa jav $t$. ol (Lev 2:6); bergin anyn üstüna jav da qojgjun anyn üstüńa levona t. ol (Lev 2:15)

-d́an: ol qalgan ol t. bolsun Aharonġa (Lev 2:3); ajyrsyn ol kohen ol t. ošol tütüsün anyn (Lev 2:9); ol qalġan ol t. Aharonġa da ulanlaryna anyn bolsun qodeš qadašim otlu qarbanlaryndan Adonajnyn (Lev 2:10); ajyrsyn ol kohen ol t. ošol tütüsün anyn (Num 5:26) -ǵa: budur ol üvŕatüv olag̉a da t. da ḥatatqa da ašamg்a da milu’imǵa da debehasyna ol šelamimnin (Lev 7:37); bir isaron öźak jumurulgan javbyla t. da bir log் jav (Lev 14:21); eksiĺarid́a tolular öźakjumurulġan javbyla t. (Num 7:13); eksilarid́a tolularöźak jumurulgan javbyla t. (Num 7:19); eksilarid́a tolular öźakjumurulgan javbyla t. (Num 7:25); passim

-j: eksitḿagin tuznu šertin Tenrijnin t. üstünd́an bar qarbanyj üstüńa juvutqun tuz (Lev 2:13)

-jnin: bar qarbanyn t. tuzbyla tuzlag்yn (Lev 2:13)

-lar: an macalar öźak halalar jumurulġanlar javbyla da juġa macalar jajgylganlar javbyla t. (Num 6:15)

-ĺari: jyllyq balalary on eki da t. alarnyn (Num 7:87); bu bolsun saja qodešindan ol qadašimnin ol ottan bar qarbanlarysajyn bar t. sajyn da bar hatatlarysajyn da bar ašamlarysajyn ki qajtarsalar maja qodeš qadašimdi saja ol da uvullaryja (Num 18:9); t. alarnyn [öźak] jumurulġan javbyla üč 'esronlar tanaj̇a da eki 'esronlar qočqarg்a qylyjyz (Num 28:20); t. alarnyn öźak jumurulgan javbyla üč esronlar ol bir tanaja (Num 28:28); t. alarnyn öźakjumurulgan javbyla (Num 29:3); passim

-ĺarijiz: bulardylar ol musaflary ki qylyjyz Adonajga moedĺarijizd́a bašqa nijetlarijizd́an da žomartlyqlaryjyzdan 'olalaryjyzsajyn da t. sajyn da nesehĺlarijizsajyn da šelamimlarijizsajyn (Num 29:39)

-ni: ošol mizbeahyn ol 'olanyn qojdu ešiginde [ miškanynyn] ohel mo'ednin da čyg்ardy anyn üstüne ošol ol 'olany da ošol ol t. ki nečik bujurdu Adonaj Mošege (Exo 40:29); keltirgin ošol ol t. ki qylynsa bulardan (Lev 2:8); juvuttu ošol ol t. da tolturdu uvučun andan (Lev 9:17); alyjyz ošol ol t. ol qalganny otlu qarbanlaryndan Adonajnyn da ašajyz any macalarjanynda ol mizbeahnyn (Lev 10:12); čygjarsyn ol kohen ošol ol 'olanyda ošol ol t. ol mizbeahta da bošatlyq qolsun anyn üčün ol kohen da arynyr (Lev 14:20); passim

-nin: budu üvratüvü ol t. juvutma any uvullary Aharonnun alnyna Adonajnyn (Lev 6:7)

-si: tava üstüńa javbyla qylynsyn qatlanġanny keltirgin any bišilirǵan t. tigimlarnin (Lev 6:14); barda t. kohennin tüǵal barysy tütatilsin ašalmasyn (Lev 6:16); t. anyn eki 'esronlar öźak jumurulgan javbyla (Lev 23:13); da t. anyn da nesehi kečinḿakḱa köŕa (Num 15:24); t. kibik ol ertanbylanyn (Num 28:8); passim 
-sidi: bermásin anyn üstüna levona ki t. künülamaklarnin ol t. saġynčlyqnyn (Num 5:15); bersin uvučlary üstüna anyn ošol tirkisin ol saġynčlyqnyn t. ol künülaḿaklarnin (Num 5:18)

-sin: ki nečik juvutsaj t. bikurlarnyn Adonajga kolos ütülǵan ottajyrtqan kurpa juvutqun ošolt. bikurlaryjnyn (Lev 2:14); bersin uvučlaryüstüńa anyn ošolt. olsag்ynčlyqnyn tirkisidi ol künülamiaklarnin (Num 5:18); alsyn ol kohen qolundan ol qatynnyn ošol $t$. (Num 5:25); qylsyn ol kohen ošol t. (Num 6:17)

-sind́an: bašqa ol hammešalik oladan da t. anyn bularny qylyjyz tüǵallar bolsunlar sizǵa da nesehĺarida alarnyn (Num 28:31); bašqa olasyndan oljanġajnyn da t. anyn da ol hammeśalik 'oladan da t. anyn (Num 29:6); ol hammeśalik óladan da t. da nesehlarind́an alarnyn (Num 29:10); bašqa ol hammeśalik oladan t. da nesehind́an anyn (Num 29:16)

-sińa: da ajtty Adonajġa qajyrylmag்yn t. alarnyn (Num 16:15)

\& Mod.NWKar. tirki '1. gift; 2. sacrifice; 3. sacrifice table'.

titrevük: titrevük tut- 'to shiver; to be taken hold on by shiver'

-tu: ešittiler uluslar bu ullu tamašalyqlarny qaltradylar t. t. olturuvčularyn Plešetnin (Exo 15:14)

e Mod.NWKar. titŕav́uk 'shiver', Mod.NWKar. tut- 'to hold'.

toḥta- 'to camp'

-dy: da t. ol bulut midbarynda Parannyn (Num 5:12)

-dylar: qyldylar uvullary Jisra’elnin barča nečik ki bujurdu Adonaj Mošeǵa alaj t. da alaj köčtülar har kiši uruvlarysajyn üvüsajyn atalarynyn (Num 2:34); da t. Etamda (Num 33:6); da t. alnynda Migdolnun (Num 33:7); da t. Marada (Num 33:8); dat. anda (Num 33:9); passim

-ma: jemeśa eki kün jemeśa janġaj jemeśa jyl uzaq turg்anda ol bulut ol miškan üstüńa t. anyn üstüna tohtaredilar ulanlary Jisra’elnin da köčmasedilar da kötürülǵanind́a köćaredilar (Num 9:22)

-redilar: ne orunda ki toḥtasajedi anda ol bulut anda t. ulanlary Jisra'elnin (Num 9:17); bujrug்una köŕa Adonajnyn köćaredilar ulanlary Jisra’elnin da bujrug்una köŕa Adonajnyn t. bar künlard́a ki tohtasajedi ol bulut ol miškan üstüna t. (Num 9:18)

-sajedi: ne orunda ki t. anda ol bulut anda tohtaredilar ulanlary Jisra'elnin (Num 9:17); bar künĺard́a ki t. ol bulut ol miškan üstüńa toḥtaredilar (Num 9:18); jemeśa eki kün jemeśa janġaj jemeśa jyl uzaq turġanda ol bulut ol miškan üstüńa toḥtama anyn üstüńa t. ulanlary Jisra’elnin da köčmasedilar da kötürülǵanind́a köćaredilar (Num 9:22)

-salar: köčsün ohel móed avulu ol Levililarnin ortasynda ol avullarnyn ki nečik t. alaj köčsünlar har kiši ornunda alamlarysajyn (Num 2:17)

-synlar: har kiši öz alamy qatyna belgilar byla üvüsajyn atalarynyn t. ulanlary Jisra’elnin qaršydan čüvŕa ohel mo`ed qatyna t. (Num 2:2); uruvlary uvullarynyn ol Qahatnyn 
t. janynda ol miškannyn (Num 3:29); nasisi üvünün atanyn uruvlarynyn Merarinin Curiel uvlu Avihajilnin janynda ol miškannyn t. cafon sary (Num 3:35)

-včular: ol t. kün tuvušusary mizrahsary alamy avulunun Juhudanyn čerüvlarisajyn (Num 2:3); ol t. anyn qatyna ševeti Jissaharnyn (Num 2:5); ol t. anyn qatyna ševeti Šimonnun (Num 2:12); ol t. anyn qatyna ševeti Ašernin (Num 2:27); ol t. alnynda ol miškannyn kün tuvušusary (Num 3:38); passim

e Mod.NWKar. tohta- 'to stop, to stay'.

toldur- 'to fill'

-du: t. alarny usubylajüreknin (Exo 35:35); ki t. ošol qolun alarnyn kohenliketŕa (Num 3:3)

-dular: japtylar alarny Pelištim da t. alarny topraqbyla (Gen 26:15)

-dum: t. any alhemi byla Tenrinin usbyla da aqylbyla da bilmek byla bar išni (Exo 31:3)

e Mod.NWKar. toltur- 'to fill', Mod.EKar. toldur-id.

toltur- see qol toltur-

tonlu: qara tonlu 'priest'

-larnyn: ančaq jerin ol q. t. satyn almady ki kem edi qara tonlularġa par'odan (Gen 47:22); ančaq jeri ol q. t.jalġyzlarnyn bolmady par'óga (Gen 47:24)

-suna: q. t. Midjannyn edijedi qyzlar (Exo 2:16)

-sunun: da berdi anar ošol Asnatny qyzyn Potifera'nyn q. t. Onnun qatynlyqqa (Gen 41:45); töredi anar Asnat qyzy Potifera'nyn q. t. Onnun (Gen 41:50); Asnat qyzy Potifera'nyn q. t. Onnun (Gen 46:19); Moše küteredi ošol qojun Jitronun qajnatasynyn q. t. Midjannyn (Exo 3:1)

e Mod.NWKar. kara tonlu 'monk'.

totafot 'frontlet'

-qa: bolsun belgige q[o]lujüstüne da t. közlerij arasyna ki küčlü qudrat byla čyg்ardy bizni Adonaj Micriden (Exo 13:16)

\& Heb. טוֹטֶ 'band, frontlet-band'.

töre et- töŕa et- '1. to judge; 2 . to think, to suppose'

-emen: ki bolsa alarga töre sözü kelediler maja da töre e. arasyna kišinin da arasyna dostunun (Exo 18:16)

-erediler: töre e. ošol ol ulusnu har vaḥtta ošol ol qaty söznü keltirirediler Mošege (Exo 18:26); bar ol kiči nerseni töre e. özleri (Exo 18:26)

-ijiz: zynharladym törečilerijizge [...] ajtadoġač [...] töre e. rastlyqbyla arasyna kišinin da arasyna qaryndašynyn da arasyna ġaripnin (Deu 1:16)

-kej: ajttylar alarga körgej Adonaj üstüj[üzge] da töre e. (Exo 5:21)

-kejlar: Tenrisi Avrahamnyn da Tenrisi Nahornun töre e. aramyzda (Gen 31:53)

-kin: syjlamaġyn jüzĺarin hožanyn rastlyqbyla töŕa e. dostujnu (Lev 19:15)

-kaj: töra e. Adonaj arama da araja (Gen 16:5)

-me: bersin törečilerge töre e. bu nerseni (Exo 21:22) $\Rightarrow$-ma

-medim: ajtty Jisra’el Josefke körḿa jüzlerijni töre e. (Gen 48:11) 
-ḿa: edi tanbyladan da olturdu Moše töre e. ošol ol ulusnu (Exo 18:13); ajttylar juvug்un arraq da ajttylar ol bir keldi tirilma da töŕa etti töra e. (Gen 19:9) $\Rightarrow$-me

-seler: bolsa talaš arasyna erenlernin da juvusalar ol törege da töre e. alarny (Deu 25:1) -sinler: töre e. ošol ol ulusnu harvahtta (Exo 18:22); da bolgaj bar ol ullu išni keltirsinler saja da bar ol kiči išni töre e. özleri (Exo 18:22) $\Rightarrow$-sinlar

-sinlar: töŕa e. ol ̌̌ymat arasyna ol uruvčunun da arasyna gečeǵasinin ol qannyn (Num 35:24) $\Rightarrow$-sinler

-ti: ajttylar juvugiun arraq da ajttylar ol bir keldi tirilḿa da töŕa e. töŕa etḿa (Gen 19:9)

e Mod.SWKar. tere et- 'to judge'. In the meaning 'to think, to suppose' the word is probably a calque of Pol. sadzić ' 1 . to judge, to sentence; 2 . to think, to suppose'.

töŕa et- $\Rightarrow$ töre et-

tujaqla- 'to part (the hoof)'

-jdy: ošol ol hazirni hotá t. tujaq ol da ajyrady ajyryčyn tujaqnyn da ol güvün güvünlamáaji murdardyr ol sizǵa (Lev 11:7); bar ol tuvar ki ol t. tujaq (Lev 11:26)

-jdy ese: ošol ol hazirni ḥote t. de tujaq (Deu 14:8)

-majdy: ašamajyz güvün keltirüvčülard́an jalġyz ol güvünnüda tujaqlavčulardan jalgyz ol tujaqny ošol ol tev́ani hot́a güvün keltiradi eśad́a ol da tujaq t. murdardy ol sizǵa (Lev 11:4); ošol ol krolikni hota güvün keltiradi eśad́a ol da tujaq t. murdardy ol sizǵa (Lev 11:5); ošol ol qojanny hoța güvün keltiradi eśad́a ol da tujaq t. murdardy ol sizǵa (Lev 11:6)

-majdylar: ošol ol teveni da ošol qojanny da ošol ol krolikni ḥote güvün keltiredilerde ese alar da tujaq t. murdarlardylar alar sizge (Deu 14:7)

-včulardan: tek ošol bunu ašamajyz güvün keltirüvčülard́an jalg̀yz ol güvünnüd́a $t$. jalg̀yz ol tujaqny ošol ol tev́ani hot́a güvün keltiradi eśad́a ol da tujaq tujaqlamajdy murdardy ol sizǵa (Lev 11:4); tek ošol bunu ašamajyz jalġyz keltirüvčülerden ol güvünnü da jalgyz t. ol tujaqny (Deu 14:7)

-včunu: bart. tujaq da ajyruvčunu ajyryčyn tujaqlarnyn güvün keltirüvčünü tuvardan any ašajyz (Lev 11:3); bar tuvarnyt. tujaq (Deu 14:6)

e Mod.NWKar. tujạ̣ 'hoof', Mod.SWKar. tujaklavcu 'dividing'.

tujaqlavču see tujaqla-

tumim 'Thummim'

-lerijni: Levi üčün ajtty t. da urimlerijni symarladyj ḥasid kišije Aharonġa (Lev 33:8)

-ni: bergin hošen ol mišpatqa ošol ol urimni da ošol ol t. da bolsun jüregi üstüne Aharonnun (Exo 28:30); qojdu anyn üstüna ošol ol ḥošenni da berdi ol ḥošenǵa ošol ol urimni da ošol ol $t$. (Lev 8:8)

e Heb. רִִִּים Thummim, i.e., one of the objects attached to the breastplate of the high priest'.

tusnaqqa al- 'to take on pledge; to borrow'

-massen: tusnaqqa berirsen köp hanlyqlarġa da sen $t$. a. da erklenirsen köp hanlyqlarda da sende erklenmesler (Deu 15:6)

e Mod.NWKar. tusnaḥ 'pledge', Mod.NWKar. tusnaḥ al- 'to take a security'. 
tusnaqqa ber- 'to give on pledge; to lend'

-gin: ačma ačqyn ošol qolujnu anar da tusna[q]qa b. anar jetkiligiče eksikliginin ki eksik bolsa anar (Deu 15:8)

-irsen: $t$. b. köp hạlyqlarga da sen tusnaqqa almassen da erklenirsen köp hạlyqlarda da sende erklenmesler (Deu 15:6);

e Mod.NWKar. tusnah 'pledge', Mod.NWKar. tusnah ber- 'to give a security'.

tutquč '1. pole; 2. tenon'

qondarsajjang்y üv da qylg̀yn t. čardag்yja da qojmag்yn qanlar üvüjde šeme jyg்ylyr ol

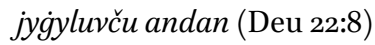

-lar: qylġyn t. šitim ag்ačlaryndan (Exo 25:13); jüzüklerind́a ol aronnun bolsunlar ol . (Exo 25:15); eki t. ol bir taḥtag்a teneštirilgenler biri birisine alaj qylġyn bar tahtalaryna ol miškannyn (Exo 26:17); qylg̀yn t. mizbeahqa šitim ag்ačlaryndan (Exo 27:6); bolsunlar ol $t$. \{ekiside $\}$ janlary üstüne ol mizbeahnyn eltkende any (Exo 27:7); eki t. ol bir taḥtaġa teneštirilgenler biri birisine (Exo 36:22); passim

-larġa: uturusuna ol beklevüčnün bolurlar ol jüzükler orunlar t. (Exo 25:27); bolsun orunlar t. eltme any alar byla (Exo 30:4); jüzükler orunlar $t$. (Exo 37:14); orunlar $t$. eltme any alarbyla (Exo 37:27); qojdu dört jüzükler dört ol učlarda ol tuč elekke orunlart. (Exo 38:5); passim

-larny: kijirgin ošol ol t. jüzüklerge janlary üstüne ol aronnun eltme ošol ol aronnu alarbyla (Exo 25:14); qylg̀yn ošol ol t. šitim aġačlaryndan (Exo 30:5); qyldy ošol ol $t$. miškanġa (Exo 36:23); kijdi ošol ol t. jüzüklerge (Exo 37:5); qyldy [ošol ol] $t$. [šitim] ag்ačaryndan (Exo 37:15); qyldy ošol ol t. šitim a jačlaryndan da qaplady alarny atlyn byla (Exo 37:28); passim

-lary: kijirilsin ošol t. anyn jüzüklerǵa (Exo 27:7)

-laryn: ošol ol aronnu da ošol t. anyn (Exo 35:12); ošol ol stolnu da ošol t. anyn (Exo 35:13); ošol mizbeahyn ol tütünün da ošol t. anyn (Exo 35:15); ošol mizbeahyn ol ólanyn da ošol ol tuč elikni ki anar ošol t. (Exo 35:16); ošol aronun ol šarajatnyn da ošol t. anyn da ošol ol kaporetni (Exo 39:35); passim

-laryna: eki tabanlar ol bir tahta tübüne eki t. da eki tabanlar ol bir tahta tübüne eki t. anyn (Exo 26:19); eki tabanlar ol bir tahta tübüne eki t. anyn da eki tabanlar ol bir tahta tübüne eki t. anyn (Exo 36:24)

e Mod.NWKar. tuthuč 'handle', Mod.SWKar. tutkuc 'railing, arm of a chair'.

tutuš- 'to congeal'

-tular: $t$. darjalar ortasynda tengiznin (Exo 15:8)

\& Mod.NWKar. tutuš- '1. (of milk) to sour; 2. to freeze'.

tuv: tuv bol- $(a b l$.) 'to be deprived of own children'

-dum: ïgej sizǵa ošol qaryndašyjyzny birni da ošol Binjaminni da men ki nečik $t$. $b$. Joseften alaj tuv boldum Šim'ondan (Gen 43:14)

-urmen: da ijermen da alyrmen seni andan nek t. b. eksijizdend́a bir künd́a ki nečik ol öltürse seni daġyn anyda öltürürĺar (Gen 27:45)

e Mod.NWKar. tuv et- 'to miscarry', Mod.SWKar. tuv et- 'to deprive of children'. 


\section{tuvduruvču 'parent'}

-larymnyn: alġyšlary atajnyn küčejdilar alġyšlary üstüne t. (Gen 49:26)

e Mod.SWKar. tuvduruvcu 'parent'.

tüneden: tüneden biri künden 'for a long time, for long'

eger süzüvčü ögüz bolsa ol t. b. k. (Exo 21:29); jemese bilinse ki süzüvčü ögüzdü ol t. $b$. k. da saqlamasa any jesisi anyn (Exo 21:36); ki dušman tüvül edi ol anar t. b. k. (Deu 19:6)

e Mod.NWKar. tunádań 'for a long time'.

tünelej: tünelej biri künlej tünelej biri künlaj 'as before, as in the past' kördü Ja'aqov ošol jüzlerin Lavannyn da muna tüvüldü anar t. b. künlej (Gen 31:2); köremen men ošol jüzlerin atajyznyn ki tüvüldü maja t. b. künlaj da Tenrisi atamnyn edi qatymda (Gen 31:5); arttyrmajyz berme bičen ulusqa kirpičleme ol kirpičlerni $t$. b. künlej alar barsynlar özleri da čöplesinler özlerińa bičen (Exo 5:7); ošol qyjasyn ol kirpičlernin ki alar qyladylar t. b. künlej qojujuz alar üstüne (Exo 5:8); neüčün tügenlemedijiz resimijizni kirpičleme t. b. künlej daġyn tünegün daġyn bügün (Exo 5:14)

e Mod.NWKar. tuńalej 'before, previously', Mod.NWKar. Kuńlej 'to this day'.

tünlej: tünlej tur- 'to get up early'

-arsiz: qonujuz da juvujuz ajaqlaryjyzny da t. t. da baryrsizjolujuzġa (Gen 19:2)

-du: t. t. Avraham ertenbylada ol orunġa ki turdu anda alnynda Adonajnyn (Gen 19:27); t. t. Ja'aqov ertenbylada da aldy ošol ol tašny (Gen 28:18); t. t. Lavan ertenbylada da öptü ulanlaryn da qyzlaryn (Gen 32:1); t. t. ertenbylada da qondardy mizbeah ol tav tübüne (Exo 24:4); t. t. Moše ertenbylada da mindi tavyna Sinajnyn (Exo 34:4)

-dular: t. t. ertenbylada da antettilar kiši qaryndašyna (Gen 26:31); t. t. tanbyladan da čýgardylar ólalar (Exo 32:6)

-gun: ajtty Adonaj Mošege t. t. ert[e]nbylada da turg்un alnynda par'onun (Exo 8:16); ajtty Adonaj Mošege t. t. ertenbylada turg்un alnynda par'onun (Exo 9:13)

\& Cf. Heb. לְהַשְכִכים 'to get up early' as the word's equivalent.

tüslü: tüslü upraq 'change of clothes'

barlaryna berdi kišiǵa t. u. da Binjaminge berdi üč jüz kümüš da beš t. u. (Gen 45:22)

e Mod.NWKar. tuślu 'various'.

tüš kör- 'to dream'

-dü: $t$. k. da muna basqyč turg்uzulġan jerǵa da bašy anyn jetadi köklerǵa (Gen 28:12); t. k. Josef tüš da anlatty qaryndašaryna özünün (Gen 37:5); t. k. özǵa tüš da qotardy any qaryndašlaryna (Gen 37:9); t. k. ekinči keret da muna jedi koloslar ösedilar bir qumašta (Gen 41:5)

-düj: nedir ol tüš ošpu ki t. k. (Gen 37:10)

-dük: ajttylar anar tüš t. k. da joralavču johtur any (Gen 40:8); t. k. tüš bir kečed́a men da olda kiši (Gen 41:11); jorasyna köra tüšünün t. k. (Gen 41:11)

-dülar: t. k. tüš eksilerid́a kiši tüšün bir kečed́a (Gen 40:5) 
-düm: tynlajyz endi ol tüšnü ki t. k. (Gen 37:6); muna t. k. tüš dag்yn muna ol qujaš da ol aj da on bir jolduzlar baš uradylar maja (Gen 37:9); ajtty par'o Josefḱa tüš t. k. da joralavču joḥtu any (Gen 41:15)

-eredi: par'o t. k. da muna turady ol Nil qatyna (Gen 41:1)

-üvčü: ki tursa ortajda navi jemese t. $k$. tüš da berse saja belgi jemese nišan (Deu 13:2); ol navi jemese t. k. ol tüšnü öltürülsün (Deu 13:6)

-üvčünün: tynlamag்yn sözlerine ol navinin jemese t. k. ol tüšnü (Deu 13:4)

\& Mod.SWKar. kisker- tisker- 'to dream'.

tüvme 'knob'

üč čaralar qošulganlar ol bir qamušta t. da japraq (Exo 25:33); üč čaralar qošulġan lar ol bir qamušta t. da japraq (Exo 25:33); t. eki ol qamušlar tübüne özünden (Exo 25:35);

t. eki ol qamušlar tübüne özünden (Exo 37:21); ̈̈č čaralar qošulġanlar ol bir qamušta t. dajapraq (Exo 37:19)

-leri: $t$. da japraqlary anyn özünden bolsunlar (Exo 25:31); t. alarnyn da qamušlary alarnyn özünden ediler (Exo 37:22); čyraqbada \{dört $\}$ čaralar qošulganlar t. anyn da japraqlary anyn (Exo 25:34); t. alarnyn da qamušlary alarnyn özünden bolsunlar (Exo 25:36); t. anyn da japraqlary anyn özünden ediler (Exo 37:17); čyraqbada \{dört\} čaralar qošulġanlar t. anyn da japraqlary anyn (Exo 37:20)

e Mod.SWKar. tuv́ma 'button'.

tüzet- 'to better'

-meśaj: eger t. ešikt́a jazyqjatady (Gen 4:7)

-śaj: eger t. išlarijni (Gen 4:7)

e Mod.SWKar. tizet- 'to better, to straighten'.

tüzü- 'to line up (troops)'

-dülar: da t. birgelerińa čerüv enišind́a ol Sidimnin (Gen 14:8)

e Mod.NWKar. tuźu- 'to organize, to put in order; to even out'. $\Rightarrow$ tüzüv

tüzüv '1. row; 2. cowshed (?)'

bergin ol t. üstüńa aruv levona (Lev 24:7)

-d́a: qojg̉un any eki tüzüvlar altyšar ḥala bolsun ol bir t. ol aruv stol üstüna (Lev 24:6)

-ler: Jissahar bazyq süvekli jatady ol eki t. arasyna (Gen 49:14) $\Rightarrow$-ĺar

-lar: qojgiun any eki t. altyšar hala bolsun ol bir tüzüvd́a (Lev 24:6) $\Rightarrow$-ler

-nün: ošol ol čyraqbany ol aruvnu ošol čyraq orunlaryn čyraqlarynyn ol t. da ošol bar savutlaryn (Exo 39:37)

e Mod.NWKar. tuźuv́ ‘. arrangement, 2. creation', Mod.NWKar. tuźu- '1. to organize, to put in order; to even out; 2 . to build'.

tyjuv 'reprimand'

-umnu: sanybyla ol künlarnin ki čajsyladyjyz ošol ol jerni qyrḥkün kün üčün jyl kün üčün jyl k[ö]türürsiz ošol žurumun günahlarijiznin qyrqjyl da bilirsiz ošol $t$. (Num 14:34) 
-un: bilijiz bügün ki tüvül ulanlaryjyz byla ki bilmediler da ki körmediler ošol t. Adonajnyn Tenrïiznin ošolu[l]lulug்un anyn ošol qudratyn ol küčlü da quvatyn anyn ol sunulgan (Deu 11:2)

e Mod.NWKar. tyj- ‘'. to reprimand, to rebuke; 2. to imprison', WKar. tyjuv 'imprisonment'.

\section{tymga 'tax'}

ajyrg்yn t. Adonajga elind́an ol čerüvnün (Num 31:28); edi ol t. Adonajga ol qojdan alty jüz da jetmiš beš (Num 31:37)

-lary: da t. alarnyn Adonajgja jetmiš eki (Num 31:38); eš́aklar otuz min da beš jüz da $t$. alarnyn Adonajga altymyš bir (Num 31:39); žany adamnyn ol alty min da t. alarnyn Adonajgia otuz eki žan (Num 31:40)

-syn: berdi Moše ošol t. terumasynyn Adonajnyn El'azarġa ol kohenǵa (Num 31:42)

\& Mod.SWKar. tymha 'customs duty'.

tynlama|q 'obedience'

-|'̇y: neginče ki kelǵaj mašijaḥ da anar bolur t. uluslarnyn (Gen 49:10)

e Mod.SWKar. tynlamak 'obedience'.

tynt- 'to inspect, to search'

-ty: da t. Lavan da tapmady ošol ol sturlaplarny (Gen 31:35); t. ulluda bašlady da kičid́a tügelledi da tabuldu ol čara qabynda Binjaminnin (Gen 44:12)

e Mod.EKar. tynt- 'to inspect; to study'.

$\mathrm{U}$

učurbyla 'unwittingly, haphazardly'

eger jürüśajiz birǵaḿa u. da kĺámaśajiz tynlama sözümáa (Lev 26:21); eger bularbylada adeplanḿaśajiz alnymda da jürüśajiz birǵaḿa u. (Lev 26:23); jürürmen ham mend́a birǵajizǵa u. da karatetarmen sizni daġyn mend́a jedi anča jazyqlaryjyz üčün (Lev 26:24); da jürüśajiz birǵaḿa u. (Lev 26:27); ham neki jürüdülar birǵaḿa u. (Lev 26:40); ham mend́a jürürmen birǵalarińa u. (Lev 26:41)

e Mod.SWKar. ucur byla 'accidentally, unwittingly'.

ulluluq 'size'

-[q]a: atadylar üvü Jisra’elnin ošol atyn anyn man da ol edi urlug்u kibik kölendranyn ulluluq $[q]$ a vale türsünü anyn aq edi (Exo 16:31)

e Mod.NWKar. ulluluḥ 'greatness', cf. Pol. wielkość '1. greatness; 2. size'.

urim 'Urim'

-lerijni: Levi üčün ajtty tumimlerijni da u. symarladyj hasid kišije Aharonġa (Lev 33:8)

-ni: bergin ḥošen ol mišpatqa ošol ol u. da ošol ol tumimni da bolsun jüregi üstüne Aharonnun (Exo 28:30); qojdu anyn üstüńa ošol ol hošenni da berdi ol hošenǵa ošol ol u. da ošol ol tumimni (Lev 8:8) 
-nin: alnynda El'azarnyn ol kohennin tursun da sorsun anar kečinḿagibyla ol u. alnynda Adonajnyn (Num 27:21)

e Heb. אוּרוּרים 'Urim, one of the objects attached to the breastplate of the high priest'.

urluqla- '1. to bear (seed); 2. to conceive (a child)'

-sa: qatyn ki u. da töraśa er ulanny (Lev 12:2)

-vču: jemiš aġačy u. urluq sizǵa bolsun jemǵa (Gen 1:29)

-včunu: berdim sizǵa ošol bar kögötnü u. urluq (Gen 1:29)

e Mod.SWKar. urlukla- 'to bear (seed)'. $\Rightarrow$ urluqlan-

urluqlan- 'to conceive (a child)'

-yr: ol qatyn [...] u. urluq (Num 5:28)

e Mod.SWKar. urlukla- 'to bear (seed)'. $\Rightarrow$ urluqla-

utok 'woof'

ol upraq jemeśa ol osnova jemeśa ol u. (Lev 13:58)

-nu: küvdürsün ošol ol upraqny jemeśa ol osnovany jemeśa ošol ol u. junda jemeśa üskülüda (Lev 13:52)

-nun: budur üvŕatüvü hastalyġynyn ol cara'atnyn ol upraqnyn jundan jemeśa üskülüd́an jemeśa osnovanyn jemeśa ol u. (Lev 13:59)

-ta: jemeśa osnovada jemeśa u. üskülünün (Lev 13:48); eger jajyldy eśa ol hastalyq upraqta jemeśa osnovada jemeśa u. (Lev 13:51); muna jajylmady ol hastalyq upraqta jemeśa osnovada jemeśa u. (Lev 13:53); eger körünśa dag்yn upraqta [je]meśa osnovada jemeśa u. (Lev 13:57)

-tan: jyrtsyn any ol upraqtan jemeśa ol teridan jemeśa ol osnovadan jemeśa ol u. (Lev 13:56)

e Russ. уток 'woof'.

uturu 'toward'

-suna: turdular andan ol erenlar da baqtylar u. Sedomnun (Gen 18:16)

e Mod.NWKar. uturu utru 'opposite'.

uvaq 'dwarfed'

jemeśa bükrü jemeśa u. jemeśa belma közünd́a jemeśa qyršang்y jemeśa qyng்yrajġan jemeśa šiškan ajipli (Lev 21:20)

\& Mod.NWKar. uvah 'small'.

uvaq: uvaq etli 'thin'

-ĺar: ašadylar ol tanalar jaman ol körümlülar da u. e. ošol jedi ol tanalarny körklü ol körümlülerni (Gen 41:4); tanalar özgelar [...] jaman körümlülar astry da u. e. körmedim alar kibik bar jerind́a Micrinin (Gen 41:19)

e Mod.NWKar. uvah 'small', Mod.NWKar. et 'flesh, meat' + deriv. suff.

uvuč 'paw'

-lary: bar ol jürüvčü u. üstüńa (Lev 11:27)

e Mod.NWKar. uvuč 'palm, hand'. 
ügütle- 'to judge'

-sinlar: qojg̉un bunda qaršysyna qaryndašlarymyn ki alardy qaryndašlaryj senind́a da ü. arasyna eksimiznind́a (Gen 31:37)

e Mod.NWKar. üǵut́la- 'to reprove'.

ündelmišs 'convocation'

burunġu künde aziz ü. da ol jedinči künde aziz ündelmiš bolsun sizge heč iš qylynmasyn alarda ančaq neki ašalady har ̌̌anġa ol jalġyz qylynsyn sizge (Exo 12:16)

e Mod.NWKar. üńda- 'to called, to convoke', Mod.EKar. ündenmiš 'gathering, assembly'. $\Rightarrow$ ünd́almüš, ünd́almiš

ünd́almišs 'convocation'

alty künlard́a qylynsyn iš da ol jedinči künd́a šabat šabaton aziz ü. heč iš qylmajyz šabatty ol Adonajġa bar olturušlaryjyzda (Lev 23:3); ol burunġu künd́a aziz ü. bolsun sizǵa heč qulluq iši qylmajyz (Lev 23:7); juvutujuz otlu qarban Adonajgja jedi künlar ol jedinči künd́a aziz ü. heč qulluq iš qylmajyz (Lev 23:8); ünd́ajiz kensisin ošpu künnün aziz ü. bolsun sizǵa heč qulluq iš qylmajyz (Lev 23:21); ol jedinči künd́a aziz ü. bolsun sizǵa heč qulluq iš qylmajyz (Num 28:25); oljedinčijanġajda burung் kününd́ajanġajnyn aziz ü. bolsun sizǵa heč qulluq iš qylmajyz (Num 29:1); onunču kününd́a ol jedinči janġajnyn ošpu aziz ü. bolsun sizǵa (Num 29:7)

e Mod.NWKar. ünd́a- 'to called, to convoke', Mod.EKar. ündenmiš 'gathering, assembly'. $\Rightarrow$ ündelmiš, ünd́almüš

ünd́almüš '1. convocation; 2. representative (convoked person)'

künü olkipurimnin ol aziz ü. bolsun sizǵa (Lev 23:27); ol burung்u künd́a aziz ü. heč qulluq iši qylmajyz (Lev 23:35); jedi künlar juvutujuz otlu qarban Adonajga ol segizinči künd́a aziz ü. bolsun sizǵa da juvutujuz otlu qarban Adonajga 'Aceretti ol heč qulluq iš qylmajyz (Lev 23:36); onbešinči kününd́a ol jedinči janġajnyn aziz ü. bolsun sizǵa heč qulluq iš qylmajyz da ḥyžlajyz ḩy̌̌ Adonajġa jedi künlar (Num 29:12)

-ĺar: sözĺagin ulanlaryna Jisra’elnin da ajtqyn alarġa mo'edĺarin Adonajnyn ki ündajiz alarny aziz ü. bulardylar alar mo'edĺarim (Lev 23:2); bulardylar mo'edlari Adonajnyn aziz ü. ki ünd́ajiz alarny vaġdalarynda alarnyn (Lev 23:4); bulardylar mo'edlari Adonajnyn ki ündajiz alarny aziz ü. juvutma otlu qarban Adonajgja 'ola da tirki debeha da nesehlar kemin künnün kününd́a (Lev 23:37)

-ĺari: bulardylar ü. ol žymatnyn nasilari ševetlarinin atalarynyn aġaraqlary minlarinin Jisra’elnin alar (Num 1:16); turdular alnynda Mošenin da éanlá ulanlaryndan Jisra’elnin eki jüz da enli nasilari žymatnyn ü. vagdanyn slavaly el (Num 16:2); ulanlary Eliavnyn Nemu'el da Datan da Aviram oldu Datan da Aviram da Aviram ü. ol žymatnyn ki qavġalaštylar Moše byla da Aharon byla (Num 26:9)

e Mod.NWKar. üńda- 'to called, to convoke', Mod.EKar. ündenmiš 'gathering, assembly'. $\Rightarrow$ ündelmiš, ünd́almiš 
üśak 'beam; staff'

bersinlar any da ošol bar savutlaryn anyn qaplavġa taḥaš terisind́an da bersinlar ol ü. üstüńa (Num 4:10); da bersinlar ol ü. üstüńa (Num 4:12); da elttilar ü. byla ökövd́a da ol narlardan da ol inžirlard́an (Num 13:23)

-ĺarin: syndyrdym ü. bojunsajyznyn (Lev 26:13)

e Mod.SWKar. isek 'pole'.

üt- 'to parch'

-ḱan: ötḿak da ü. kolos da kurpa ašamajyz kensisina dejin ošpu künnün (Lev 23:14)

^ MTkc. üt- 'to singe, to scorch', see Räsänen (1969: 524).

ütül- 'to be parched'

-ǵan: ki nečik juvutsaj tirkisin bikurlarnyn Adonajg̈a kolos ü. ottajyrtqan kurpa juvutqun ošol tirkisin bikurlaryjnyn (Lev 2:14)

e MTkc. üt- 'to singe, to scorch', see Räsänen (1969: 524).

V

vadaj 'surely, certainly'

ki bilamen ki nečik qarġasaj v. bolalyrmen vatma any (Num 22:6); ki eger qarġasaj v. bolalyrmen urušma anyn byla (Num 22:11)

\& Heb. 'Iָ 'surely, certainly'.

vahtlyq *'in readiness, ready'

ijsin qolu ašyra ol $v$. kišinin ol midbar (Lev 16:21)

e Mod.NWKar. vaht 'time' + deriv. suff. A calque of BHeb. עִ̣ת 'timely, ready' (a hapax in the Bible, Lev 16:21).

vezir 'officer'

-leri: ačuvlandy par'o eki v. üstüńa (Gen 40:2)

-lerind́an: sorduv. paronun (Gen 40:7)

e Mod.NWKar. vezir 'vizier'.

vole '(anat.) crop'

-sin: ketersin ošolv. anyn junubyla da tašlasyn any janynda ol mizbeaḥnyn kün tuvušusary ornuna ol külnün (Lev 1:16)

\& Pol. wole '(anat.) crop'.

\section{$\mathbf{Z}$}

zavly 'a person that has a discharge'

töśak ki jatsa anyn üstüńa ol z. murdar bolsun (Lev 15:4); ol z. juvsun upraqlaryn (Lev 15:6); ki tükürśa ol z. kiši aruv kiši üstüńa da juvsun upraqlaryn (Lev 15:8); bar ol atlanmaq kiatlansa anyn üstüna olz. murdarbolsun (Lev 15:9); barča neki tijśa anar ol z. da 
qollaryn juvmasa suvbyla da juvsun upraqlaryn da juvsun gufun suvbyla da murdar bolsun (Lev 15:11); passim

-ny: sürsünlar ol avuldan bar cara'atlyny da bar z. (Num 5:2)

-nyn: ol z. zavlyg்ynda erkaknin da qatyn kišinin da kišinin ki jatsa murdar qatyn byla (Lev 15:33)

\& Heb. זָ 'a running discharge' + Kar. deriv. suff. $\Rightarrow$ zavlyq

zavly $\mid \mathbf{q}$ 'discharge'

-|g்ynda: bu bolsun murdarlyg்y anyn z. siliǵaj bolsa gufundan z.jemeśa beklaśaj gufun anyn zavlyg்yndan murdarlyg்ydy anyn ol (Lev 15:3); ol zavlynyn z. erkaknin da qatyn kišinin da kišinin ki jatsa murdar qatyn byla (Lev 15:33)

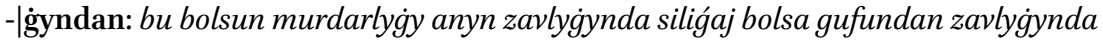
jemeśa beklaśaj gufun anyn z. murdarlyg்ydy anyn ol (Lev 15:3); ki arynsa ol zavly z. da sansyn özüna jedi künlar arynmaq vahtyndan (Lev 15:13); bošatlyq qolsun anyn üčün ol kohen alnynda Adonajnyn z. (Lev 15:15); eger arynsa z. da sansyn özüna jedi künlar (Lev 15:28); bošatlyq qolsun anyn üčün ol kohen alnynda Adonajnyn z. murdarlyg்ynyn (Lev 15:30); passim

$\ell$ Heb. זָז 'a running discharge' + Kar. deriv. suff. $\Rightarrow$ zavly

zyndan: zyndan üv $\sim$ zyndan üvü 'prison'

-d́a: orunga ki tutqunlary ol bijnin tutqunlangan edilar da edi anda ol z. üvd́a (Gen 39:20); berdi ag்arag்y ol zyndan üvünün erkine Josefnin ošol bar ol tutqunlang்anlarny ki ol z. üvd́a (Gen 39:22); ki tutqunlangan edilar ol z. üvd́a (Gen 40:5)

-ǵa: aldy biji Josefnin any da berdi any ol z. üvǵa orunġa ki tutqunlary ol bijnin tutqunlanġan edilar (Gen 39:20); berdi alarny saqlavyna üvünün ag்arag்ynyn ol qasapčylarnyn ol z. üvǵa (Gen 40:3)

-nün: közlerind́a ag்araġynyn ol z. üvünün (Gen 39:21); berdi ag்arag்y ol z. üvünün erkine Josefnin ošol bar ol tutqunlanganlarny (Gen 39:22); ag்arag்y ol z. üvünün baqmasedi (Gen 39:23)

e Mod.NWKar. zindan zyndan 'prison'. $\Rightarrow$ syndan, syndan üv

\section{$\check{\mathbf{Z}}$}

žalle- 'to regret; to sympathise'

-megin: hajifsünmesin közüj anyn üstüne da ž. da jašyrmag்yn günehin anyn (Deu 13:9)

e Pol. żatować 'to regret; to sympathise', Mod.NWKar. želle- žejle- žeyla- 'to regret, to sympathise', Mod.NWKar. žal 'pity'.

žubra 'wisent'

-ny: soġagny da jürnü da bojvolnu da pole qojun da dišonnu da lośnu da ž. (Deu 14:5)

e Pol. $\dot{z} u b r$ 'European bison, wisent', Russ. зубp id., Ukr. зубр id. 